\title{
Resource Allocation in Correlated MIMO Systems
}

\author{
by \\ Francisco Cano Broncano
}

Submitted to the CAPD of the School of Telecommunications, Systems and Engineering in partial fulfillment of the requirements for the degree of Ph.D. in Systems and Services Engineering for the Informtion Society at the

UNIVERSIDAD POLITECNICA DE MADRID

September 2016

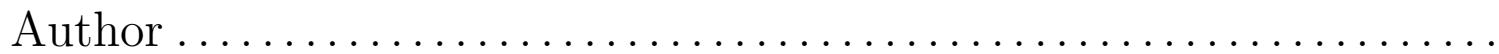

School of Telecommunications, Systems and Engineering

October 1, 2016

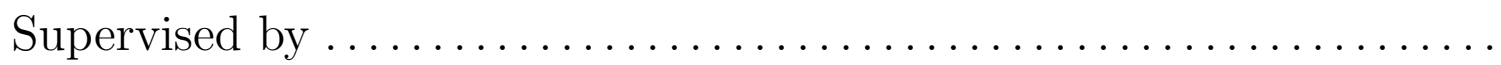

Dr. César Benavente Peces

School of Telecommunications, Systems and Engineering

Thesis Supervisor

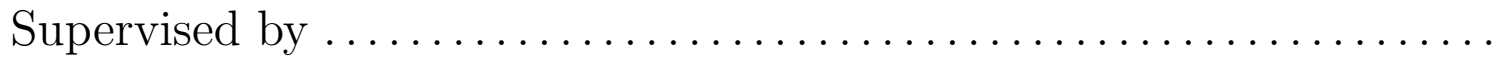

Dr. Francisco Javier Ortega González School of Telecommunications, Systems and Engineering Thesis Supervisor 


\title{
Resource Allocation in Correlated MIMO Systems
}

\author{
by
}

\author{
Francisco Cano Broncano
}

\author{
Submitted to the School of Telecommunications, Systems and Engineering \\ on October 1, 2016, in partial fulfillment of the \\ requirements for the degree of \\ Ph.D. in Systems and Services Engineering for the Informtion Society
}

\begin{abstract}
Antennas proximity produces the antennas correlation effect which impacts over the Multiple-Input Multiple-Output (MIMO) system performance. This PhD thesis evaluate the receiver-side antennas correlation effect and outputs the appropriate antennas set-up, showing simulation results for a $(4 \times 4)$ correlated MIMO channel with linear and non-linear uniformly spaced antennas. Therefore, optimal and sub-optimal resource allocation techniques for MIMO systems are compared. Bit and power allocation techniques have been investigated in order to find out optimal algorithms to achieve the best bit-error rate (BER) performance. An optimal power allocation (PA) technique based on Lagrange multiplier method is compared against a sub-optimal PA based on an equal-SNR for uncorrelated and correlated channel profiles. Following the sub-optimal strategies, a novel iterative bit- and power allocation (IBPA) approach has been developed when transmitting a given bit/s/Hz data rate over a correlated frequency non-selective $(4 \times 4)$ MIMO channel. The iterative resources allocation algorithm developed in this investigation is aimed at the achievement of the minimum BER in a correlated MIMO communication system. In order to achieve this goal, the available bits are iteratively allocated in the MIMO active layers which present the minimum transmit power requirement per time slot. Finally, this single-user MIMO system has been extended to multi-user MIMO system.
\end{abstract}


"It seems that the harder I work, the luckier I am."

Thomas Jefferson 
This doctoral thesis has been examined by a Committee as follows:

Professor

Chairman, Thesis Committee

University

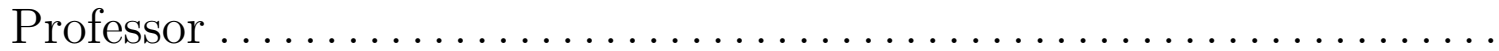

Member, Thesis Committee

University

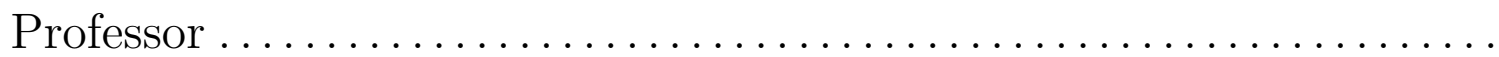

Member, Thesis Committee

University

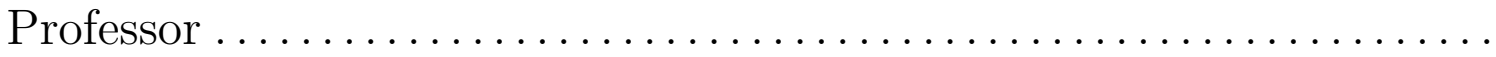

Member, Thesis Committee

University

Professor

Member, Thesis Committee

University 


\section{Contents}

1 Introduction $\quad 22$

1.1 State-of-the-art . . . . . . . . . . . . . . . . 22

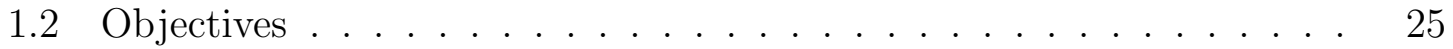

2 MIMO Channel $\quad 29$

2.0.1 Wireless Radio Channel . . . . . . . . . . . . . . . . . . . . . 29

2.0.2 Model of the Wireless Channel . . . . . . . . . . . . . . . 33

2.1 Antennas Correlation in MIMO Systems . . . . . . . . . . . . . 47

2.1.1 Antennas Correlation Characterization between two Adjacent Receive Antennas . . . . . . . . . . . . . . . 47

2.1.2 Antennas Correlation in Scattered MIMO Environments . . . 49

2.1.3 Antennas correlation on a $(4 \times 4)$ MIMO link . . . . . . 59

2.2 Chapter Conclusions . . . . . . . . . . . . . . . 66

3 Signal Processing in MIMO Systems $\quad 67$

3.1 Singular Value Decomposition . . . . . . . . . . . . . . . 67

3.2 SDM MIMO Model and Quality Criteria . . . . . . . . . . . 72

3.2.1 BER Performance in MIMO Systems . . . . . . . . . . . . . . 73

3.3 Power Allocation . . . . . . . . . . . . . . . 79

3.3.1 Equal-SNR Power Allocation ............. 81

3.3.2 Water-filling . . . . . . . . . . . . . . 88

3.3.3 Iterative Bit and Power Allocation . . . . . . . . . . . . . . . 94

3.4 Geometric Mean Decomposition . . . . . . . . . . . . . 105 
3.5 Tomlinson-Harashima Precoding . . . . . . . . . . . . . . . . . . . 108

3.6 Comparison SVD-GMD . . . . . . . . . . . . . . . . . . . . 114

3.7 Chapter Conclusions . . . . . . . . . . . . . . . . . . . . . . 117

4 Multi-user MIMO Systems 121

4.1 SVD-aided Multi-user MIMO System Model . . . . . . . . . . . . . 121

4.2 GMD-aided Multi-User MIMO System Model . . . . . . . . . . . . . 129

4.3 Chapter Conclusions . . . . . . . . . . . . . . . . . . . . . . . 134

5 Conclusions 136

5.1 Achievements . . . . . . . . . . . . . . . . . 136

5.2 Contributions . . . . . . . . . . . . . . . . . 137 


\section{List of Figures}

1-1 Capacity comparison between SISO $\left(n_{\mathrm{T}}=n_{\mathrm{R}}=1\right)$ and $\operatorname{MIMO}\left(n_{\mathrm{T}}=\right.$ $n_{\mathrm{R}}=4$ ) frequency non-selective system . . . . . . . . . . . . 23

2-1 Time model of SISO frequency non-selective channel . . . . . . . . . 34

2-2 Time model of SISO frequency selective channel . . . . . . . . . . 35

2-3 Time model of SISO baseband equivalent model . . . . . . . . . . . . 36

2-4 Time model of SISO baseband equivalent model simplified . . . . . . 37

2-5 Discrete model of SISO frequency non-selective channel . . . . . . . . 37

2-6 Discrete model of SISO frequency selective channel . . . . . . . . . . 38

2-7 Time model of MIMO frequency non-selective channel . . . . . . . . . 39

2-8 Discrete model of MIMO frequency non-selective channel . . . . . . . 41

2-9 Discrete model of MIMO frequency selective channel . . . . . . . . . 43

2-10 Antennas physical set-up: one transmitter-side antenna and four linearly distributed and equally spaced receiver-side antennas . . . . . . 50

2-11 Antennas physical set-up: one transmitter-side antenna and four nonlinearly distributed and equally spaced receiver-side antennas . . . . .

2-12 CCDF of the layer-specific distribution for uncorrelated frequency nonselective $(4 \times 4)$ MIMO channel with linear spatial distribution . . . . 
2-13 CCDF of the layer-specific distribution for uncorrelated (solid line), the weak correlated (dashed line) and the strong correlated (dotted line) frequency non-selective $(4 \times 4)$ MIMO channel with linear spatial distribution. The established parameters in the simulation are $\phi=30^{\circ}$ $\mathrm{rad}, \sigma_{\xi}=1$ and $d_{\lambda}=1$ in order to simulate a weak correlation (CM-1), and $d_{\lambda}=0.25$ for strong correlation (CM-2). A non-correlated one is set for comparison reasons $(\mathrm{CM}-3) \ldots \ldots \ldots \ldots$

2-14 CCDF of the layer-specific distribution for uncorrelated (solid line), the weak correlated (dashed line) and the strong correlated (dotted line) frequency non-selective $(4 \times 4)$ MIMO channel with linear spatial distribution. The established parameters in the simulation are $\phi=30^{\circ}$ $\mathrm{rad}, \sigma_{\xi}=1$ and $d_{\lambda}=1$ in order to simulate a weak correlation (CM-1), and $d_{\lambda}=0.25$ for strong correlation (CM-2). A no correlated one is set for comparison reasons $(\mathrm{CM}-3) \ldots \ldots \ldots \ldots$

2-15 CCDF of the layer-specific distribution for uncorrelated (solid line), the weak correlated (dashed line) and the strong correlated (dotted line) frequency non-selective $(4 \times 4)$ MIMO channel with non-linear distribution. The established parameters in the simulation were $\phi=$ $90^{\circ} \mathrm{rad}, \sigma_{\xi}=1$ and $d_{\lambda}=1$ in order to simulate a weak correlation $(\mathrm{CM}-1)$ and $d_{\lambda}=0.25$ for strong correlation $(\mathrm{CM}-2) \ldots \ldots \ldots$

2-16 CCDF of the layer-specific distribution for the weak correlated (dashed line) with linear distribution and the weak correlated (dotted line) with non-linear distribution for a frequency non-selective $(4 \times 4)$ MIMO channel. The established parameters in the simulation were $\phi=30^{\circ}$ $\operatorname{rad}, \sigma_{\xi}=1$ and $d_{\lambda}=1$ in order to simulate a weak correlation and $d_{\lambda}=0.25$ for strong correlation $\ldots \ldots \ldots \ldots \ldots$ 
2-17 CCDF of the layer-specific distribution for the strong correlated (dashed line) with linear distribution and the strong correlated (dotted line with non-linear distribution for a frequency non-selective $(4 \times 4)$ MIMO channel. The established parameters in the simulation were $\phi=30^{\circ}$ $\operatorname{rad}, \sigma_{\xi}=1$ and $d_{\lambda}=1$ in order to simulate a weak correlation and $d_{\lambda}=0.25$ for strong correlation . . . . . . . . . . . . . .

2-18 Capacity comparison between uncorrelated and correlated (linear and non-linear distribution) in a frequency non-selective $(4 \times 4)$ MIMO system 66

3-1 PDF (probability density function) of the comparison between the smallest $\sqrt{\xi^{(4)}}$ and the largest singular value $\sqrt{\xi^{(1)}}$ with linear spatial distribution with weak (CM-1) and strong (CM-2) correlation, and both are compared with no correlation one as a reference (CM-3) in a frequency non-selective $(4 \times 4)$ MIMO system . . . . . . . . . . . . 70

3-2 PDF (probability density function) of the comparison between the smallest $\sqrt{\xi^{(4)}}$ and the largest singular value $\sqrt{\xi^{(1)}}$ with non-linear spatial distribution with weak (CM-1) correlation compared with no correlation one as a reference $(\mathrm{CM}-3)$ in a frequency non-selective $(4 \times 4)$ MIMO system . . . . . . . . . . . . . . . . . 70

3-3 PDF (probability density function) of the comparison between the smallest $\sqrt{\xi^{(4)}}$ and the largest singular value $\sqrt{\xi^{(1)}}$ with linear and non-linear spatial distribution with weak (CM-1) correlation in a frequency non-selective $(4 \times 4)$ MIMO system . . . . . . . . . . . . 71

3-4 Layer-specific system model . . . . . . . . . . . . . . . . . 75

3-5 Bit-error probability weakly correlated with linear spatial distribution when using the transmission modes introduced in Table 3.1 and transmitting $8 \mathrm{bit} / \mathrm{s} / \mathrm{Hz} \ldots \ldots \ldots \ldots$. . . . . . . . . . . . 78

3-6 Bit-error probability strongly correlated with linear spatial distribution when using the transmission modes introduced in Table 3.1 and transmitting $8 \mathrm{bit} / \mathrm{s} / \mathrm{Hz} \ldots \ldots \ldots \ldots$ 
3-7 Layer-specific system model including power allocation parameter . .

3-8 BER (Bit-error-rate) curves of $(4,4,4,4)$ QAM transmission mode comparing Optimum solution and Equal-SNR Power Allocation effect over (CP-1) (solid line) and (CP-2) (dotted line) . . . . . . . . . . . 84

3-9 BER (Bit-error-rate) curves of $(16,4,4,0)$ QAM transmission mode comparing Equal-SNR Power Allocation effect over (CP-1) (solid line) and $(\mathrm{CP}-2)($ dotted line $) \ldots \ldots \ldots \ldots$

3-10 BER (Bit-error-rate) curves of $(16,16,0,0)$ QAM transmission mode comparing Equal-SNR Power Allocation effect over (CP-1) (solid line) and $(\mathrm{CP}-2)($ dotted line $) \ldots \ldots \ldots$

3-11 BER with equal-SNR PA (dotted line) and without PA (solid line) when using the transmission modes introduced in Table 3.3 and transmitting 8 bit/s/Hz over frequency non-selective $(4 \times 4)$ MIMO channels with weak antenna correlation . . . . . . . . . . . . .

3-12 BER with equal-SNR PA (dotted line) and without PA (solid line) when using the transmission modes introduced in Table 3.3 and transmitting 8 bit/s/Hz over frequency non-selective $(4 \times 4)$ MIMO channels with strong antenna correlation . . . . . . . . . . . .

3-13 PDF (probability density function) of choosing different transmission modes when using the transmission modes introduced in Table 3.3 and transmitting $8 \mathrm{bit} / \mathrm{s} / \mathrm{Hz}$ over uncorrelated, weakly correlated (CM-1) and strongly correlated $(\mathrm{CM}-2)$ frequency non-selective $(4 \times 4) \mathrm{MIMO}$

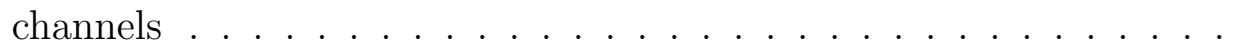

3-14 Water-filling diagram for a fixed time slot . . . . . . . . . .

3-15 Layer-specific system model including power allocation parameter with water-filling technique . . . . . . . . . . . . 
3-16 BER with Water-filling (dashed line) compared to BER curves with equal-SNR PA (dotted line) and without PA (solid line) when using the transmission modes introduced in Table 3.1 and transmitting 8 bit/s/Hz over frequency non-selective $(4 \times 4)$ MIMO channels with weak antenna correlation . . . . . . . . . . . . . . .

3-17 BER with Water-filling (dashed line) compared to BER curves with equal-SNR PA (dotted line) and without PA (solid line) when using the transmission modes introduced in Table 3.1 and transmitting 8 bit/s/Hz over frequency non-selective $(4 \times 4)$ MIMO channels with strong antenna correlation . . . . . . . . . . . . . . . . . . . 93

3-18 Iterative Bit- and Power Allocation scheme at a fixed data rate . . . . 98

3-19 Iterative Bit- and Power Allocation scheme at a variable data rate . .

3-20 BER with IBPA fixed data rate algorithm (dashed line) compared to BER curves with equal-SNR PA (dotted line) and without PA (solid line) when using the transmission modes introduced in Table 3.1 and transmitting $8 \mathrm{bit} / \mathrm{s} / \mathrm{Hz}$ over frequency non-selective $(4 \times 4)$ MIMO channels with weak antenna correlation . . . . . . . . . . . . 102

3-21 BER with IBPA fixed data rate algorithm (dashed line) compared to BER curves with equal-SNR PA (dotted line) and without PA (solid line) when using the transmission modes introduced in Table 3.3 and transmitting $8 \mathrm{bit} / \mathrm{s} / \mathrm{Hz}$ over frequency non-selective $(4 \times 4) \mathrm{MIMO}$ channels with strong antenna correlation . . . . . . . . . . . 102

3-22 BER with IBPA variable data rate algorithm (dashed line) compared to BER curves with equal-SNR PA (dotted line) and without PA (solid line) when using the transmission modes introduced in Table 3.1 and transmitting $8 \mathrm{bit} / \mathrm{s} / \mathrm{Hz}$ over frequency non-selective $(4 \times 4) \mathrm{MIMO}$ channels with weak antenna correlation . . . . . . . . . . . 103 
3-23 BER with IBPA variable data rate algorithm (dashed line) compared to BER curves with equal-SNR PA (dotted line) and without PA (solid line) when using the transmission modes introduced in Table 3.3 and transmitting $8 \mathrm{bit} / \mathrm{s} / \mathrm{Hz}$ over frequency non-selective $(4 \times 4) \mathrm{MIMO}$ channels with strong antenna correlation . . . . . . . . . . 103

3-24 Comparison PA techniques developed. IBPA at a fixed data rate, IBPA at a variable data rate, Water-filling and Equal-SNR PA when transmitting an average data throughput of $8 \mathrm{bit} / \mathrm{s} / \mathrm{Hz}$ over frequency nonselective $(4 \times 4)$ weak correlated MIMO channels . . . . . . . . . . . 104

3-25 Pre- and post processing GMD model for MIMO systems . . . . . . . 106

3-26 Simplified GMD model for MIMO systems after signal processing . 106

3-27 CCDF of the layer-specific distribution for uncorrelated frequency nonselective $(4 \times 4)$ GMD-aided MIMO channel . . . . . . . . . . . . 108

3-28 PDF of the comparison between the effect of the uncorrelation and the strong correlation in a frequency non-selective $(4 \times 4)$ GMD-aided MIMO system with $L_{\mathrm{a}}=3 \ldots \ldots$. . . . . . . . . . . . . . 109

3-29 CCDF of the comparison between the effect of the uncorrelation and the strong correlation in a frequency non-selective $(4 \times 4)$ GMD-aided MIMO system with $L_{\mathrm{a}}=3 \ldots \ldots$. . . . . . . . . . . 109

3-30 BER comparison of the uncorrelated frequency non-selective $(4 \times 4)$ GMD-aided MIMO system with equal-SNR PA technique (dotted lines) and without (solid lines) assuming perfect interference cancellation .

3-31 BER comparison of the correlated frequency non-selective $(4 \times 4)$ GMDaided MIMO system with equal-SNR PA technique (dotted lines) and without (solid lines) assuming perfect interference cancellation . . . . 110

3-32 Successive Interference Cancellation model for MIMO systems in the receiver side . . . . . . . . . . . . . . . . . . . 111

3-33 Tomlinson-Harashima precoding model for MIMO systems . . . . . . 112 
3-34 BER comparison between the Perfect Interference Cancellation technique and the Successive Interference Cancellation in the receiver side for $(4,4,4,4)$ uncorrelated frequency non-selective $(4 \times 4)$ GMD-aided MIMO channel . . . . . . . . . . . . . . . . . .

3-35 BER comparison between the Perfect Interference Cancellation technique and THP for $(16,16,0,0)$ uncorrelated frequency non-selective $(4 \times 4)$ GMD-aided MIMO channel . . . . . . . . . . . . . . . . 114

3-36 Constellation diagram from transmit symbols with GMD technique and Tomlinson-Harashima precoding in the transmission side when the $(16,16,0,0)$ mode is transmitted over frequency non-selective $(4 \times 4)$ MIMO channels . . . . . . . . . . . . . . . . .

3-37 Constellation diagram from transmit symbols with GMD technique and Tomlinson-Harashima precoding in the transmission side when the $(16,16,0,0)$ mode is transmitted over frequency non-selective $(4 \times 4)$ MIMO channels with correlation . . . . . . . . . . . . 115

3-38 CCDF of the 3-active-layer-specific distribution for uncorrelated frequency non-selective $(4 \times 4)$ GMD-aided MIMO channel compared to the SVD-aided MIMO channel . . . . . . . . . . . . .

3-39 BER curves with GMD technique (dashed line) assuming perfect interference cancellation compared to BER curves with SVD (solid line) when using the transmission modes introduced in the legend with equal-SNR PA and transmitting $8 \mathrm{bit} / \mathrm{s} / \mathrm{Hz}$ over frequency non-selective $(4 \times 4)$ MIMO channels without antenna correlation . . . . . . . . 117

3-40 BER curves with GMD technique (dashed line) assuming perfect interference cancellation compared to BER curves with SVD (solid line) when using the transmission modes introduced in the legend with equal-SNR PA and transmitting $8 \mathrm{bit} / \mathrm{s} / \mathrm{Hz}$ over frequency non-selective $(4 \times 4)$ MIMO channels with antenna correlation . . . . . . . . 118 
3-41 BER curves with GMD technique (dashed line) assuming perfect interference cancellation compared to BER curves with SVD (solid line) and the best transmission mode per each time slot with equal-SNR $\mathrm{PA}$ and transmitting $8 \mathrm{bit} / \mathrm{s} / \mathrm{Hz}$ over frequency non-selective $(4 \times 4)$ MIMO channels without antenna correlation . . . . . . . . . . . 119

3-42 BER curves with GMD technique (dashed line) assuming perfect interference cancellation compared to BER curves with SVD (solid line) and the best transmission mode per each time slot with equal-SNR $\mathrm{PA}$ and transmitting $8 \mathrm{bit} / \mathrm{s} / \mathrm{Hz}$ over frequency non-selective $(4 \times 4)$ MIMO channels with antenna correlation . . . . . . . . . . . . . 120

4-1 Multi-user MIMO system model . . . . . . . . . . . . . . . . . . . . 121

4-2 Multi-user MIMO system model . . . . . . . . . . . . . . . . . . . . 122

4-3 Multi-user MIMO system model after SVD-decomposition . . . . . . 124

4-4 Multi-user MIMO system model with ZF-preprocessing . . . . . . . . 124

4-5 User-specific BERs comparison when using the transmission modes introduced in Table 3.1 and transmitting 8 bit/s/Hz over frequency non-selective uncorrelated SVD-aided Multi-user MIMO system . . . 128

4-6 User-specific BERs comparison when using the transmission modes introduced in Table 3.1 and transmitting 8 bit/s/Hz over frequency non-selective correlated SVD-aided Multi-user MIMO system . . . . . 128

4-7 MU-MIMO system model with ZF-preprocessing . . . . . . . . . . . . 130

4-8 User-specific BERs comparison when using the transmission modes introduced in Table 3.1 and transmitting $8 \mathrm{bit} / \mathrm{s} / \mathrm{Hz}$ over frequency non-selective uncorrelated GMD-aided MU-MIMO systems . . . . . . 132

4-9 User-specific BERs comparison when using the transmission modes introduced in Table 3.1 and transmitting 8 bit/s/Hz over frequency non-selective correlated GMD-aided MU-MIMO systems . . . . . . . 133 
4-10 User-specific BERs comparison between the SVD-aided and GMDaided MIMO system when using the transmission modes $(16,16,0,0)$ and $(4,4,4,4)$ and transmitting $8 \mathrm{bit} / \mathrm{s} / \mathrm{Hz}$ over frequency non-selective uncorrelated Rayleigh channels . . . . . . . . . . . . . . . . 135 


\section{List of Tables}

2.1 Channel Profiles for Linear Distribution _ . . . . . . . . . . 60

2.2 Channel Profiles for Non-linear Distribution . . . . . . . . . . 61

3.1 Investigated QAM transmission modes $\ldots \ldots \ldots \ldots . \ldots 77$

3.2 Investigated channel profiles assuming a $(4 \times 4)$ MIMO system $\ldots . .83$

3.3 Investigated PA-QAM transmission modes . . . . . . . . . . 83

3.4 Investigated channel profiles assuming a $(4 \times 4)$ MIMO system with IBPA fixed data rate at $E_{\mathrm{s}} / N_{0}=20 \mathrm{~dB} \ldots \ldots \ldots \ldots$

3.5 Investigated channel profiles assuming a $(4 \times 4)$ MIMO system with IBPA variable data rate at $E_{\mathrm{s}} / N_{0}=20 \mathrm{~dB} \ldots \ldots \ldots \ldots$

4.1 Simulation parameters for multi-user MIMO system . . . . . . . . 127 


\title{
Abbreviations
}

\author{
AM Adaptive Modulation \\ BER Bit Error Rate \\ CCDF Complementary Cumulative Distribution Function \\ i.i.d Independent Identically Distributed \\ IBPA Iterative Bit Power Allocation \\ ISI Inter Symbol Interference \\ LAN Local Area Network \\ LOS Line Of Sight \\ LTE Long Term Evolution \\ NLOS Non Line Of Sight \\ MIMO Multiple Input Multiple Output \\ MU-MIMO Multi-user Multiple Input Multiple Output \\ PA Power Allocation \\ PCSI Perfect Channel State Information \\ PSK Phase Shifting Keying \\ QAM Quadrature Amplitude Modulation \\ SISO Single Input Single Output \\ SNR Signal Noise Rate \\ SVD Singular Value Decomposition \\ WiMAX Worldwide Interoperability Microwave Access
}




\title{
Symbols
}

\author{
$A(d) \quad$ Path attenuation depending on distance \\ $a[k] \quad$ Modulated transmit data \\ $B_{\mathrm{L}} \quad$ Lagrange boundary data \\ $b^{(\mu)} \quad$ Number of transmitted bits per layer \\ $b_{\mathrm{e}}^{(\mu)} \quad$ Number of error bits per layer \\ D Orthogonal matrix whose columns are the eigenvalues of $\mathbf{H}^{\mathrm{T}} \cdot \mathbf{H}$ \\ $d^{(1)} \quad$ Distance respect antenna $\sharp 1$ \\ $d_{\lambda} \quad$ Distance measured in wavelength \\ $E_{\mathrm{s}} \quad$ Energy per symbol \\ $f \quad$ Frequency \\ G Uncorrelated channel matrix \\ $G_{\mathrm{RX}} \quad$ Receive antenna gain \\ $G_{\mathrm{TX}} \quad$ Transmit antenna gain \\ $G_{\mathrm{k}}^{(11)}(f) \quad$ Frequency response. Fourier transform of $g_{\mathrm{k}}^{(11)}(t)$ \\ $g_{\mathrm{R}}^{(11)}(t) \quad$ Receiver filter \\ $g_{\mathrm{T}}^{(11)}(t) \quad$ Transmitter filter \\ $g_{\mathrm{k}}^{(11)}(t) \quad$ Impulse response \\ $g_{\mathrm{k} \nu}^{(11)}(t) \quad$ Impulse response for frequency selective channel \\ H Channel matrix \\ $\mathbf{H}^{(11)}[k] \quad$ Discrete frequency selective channel matrix \\ $\mathbf{H}^{(\mu \nu)}[k] \quad$ Discrete MIMO frequency selective channel matrix \\ $h^{(11)}(t) \quad$ Baseband channel
}


$h^{(\mu \nu)}(t) \quad$ MIMO baseband channel

$h_{0} \quad$ Complex Gaussian random variable

$h_{0}^{(11)}[k] \quad$ Discrete non-frequency selective channel element

$h_{\mathrm{L}}^{(11)}[k] \quad$ Discrete frequency selective channel $L$ element

$L \quad$ Number of delay taps

$L_{\mathrm{a}} \quad$ Number of active layers

$M^{(\mu)} \quad$ Index modulation per layer

$\mathbf{n}[k] \quad$ Discrete MIMO non-frequency selective noise vector

$\mathbf{n}^{(1)}[k] \quad$ Discrete frequency selective noise vector

$\mathbf{n}^{(\mu)}[k] \quad$ Discrete MIMO frequency selective noise vector

$n^{(1)}[k] \quad$ Discrete baseband noise element

$n^{(11)}(t) \quad$ SISO baseband noise

$n^{(\mu)}(t) \quad$ MIMO baseband noise

$n_{\mathrm{N}_{\mathrm{o}}}^{(1)}[k] \quad$ Discrete frequency selective noise

$n_{\mathrm{R}} \quad$ Number of receiver antennas

$n_{\mathrm{T}} \quad$ Number of transmit antennas

$P_{\mathrm{b}}^{(\mu)} \quad$ Bit probability per layer

$P_{\mathrm{BER}}^{(\mu)} \quad$ Overall bit-error-rate probability

$P_{\text {BER }}^{(\mu)} \quad$ Bit-error-rate probability per layer

$P_{\mathrm{BER}} \quad$ Overall bit-error-rate probability with power allocation

$P_{\mathrm{BER}_{\mathrm{PA}}}^{(\mu)} \quad$ Bit-error-rate probability per layer with power allocation

$P_{\mathrm{BER}_{\mathrm{wf}}} \quad$ Overall bit-error-rate probability with water-filling technique

$P_{\mathrm{BER}_{\mathrm{wf}}}^{(\mu)} \quad$ Bit-error-rate probability per layer with water-filling technique

$P_{\mathrm{s}} \quad$ Transmit power

$P_{\mathrm{s}}^{(\mu)} \quad$ Transmit power per layer

$P_{\mathrm{SPA}} \quad$ Transmit power with power allocation

$P_{\mathrm{SPA}}^{(\mu)} \quad$ Transmit power with power allocation per layer 
$p(\xi) \quad$ Probability distribution function

$\sqrt{p^{(\mu)}} \quad$ Power allocation factor per layer

$\sqrt{p_{\mathrm{wf}}^{(\mu)}} \quad$ Power allocation factor per layer by water-filling technique

$\mathbf{R}_{\mathrm{HH}} \quad$ Overall correlation matrix

$\mathbf{R}_{\mathrm{RX}} \quad$ Receive correlation matrix

$\mathbf{R}_{\mathrm{TX}} \quad$ Transmit correlation matrix

$r \quad$ Amplitude of the probability density function

S Orthogonal matrix whose columns are the eigenvalues of $\mathbf{H} \cdot \mathbf{H}^{\mathrm{T}}$

$S_{\mathrm{w}} \quad$ Power transmit threshold in water-filling technique

$T_{\mathrm{s}} \quad$ Symbol period

$t \quad$ Time

$U_{\mathrm{A}} \quad$ Half vertical eye opening

$U_{\mathrm{APA}} \quad$ Half vertical eye opening with power allocation

$U_{\mathrm{R}} \quad$ Noise power per quadrature component

$u_{\mathrm{e}}^{(1)}(t) \quad$ Received signal

$u_{\mathrm{k}}^{(1)}(t) \quad$ Transmitted signal through the channel

$w^{(1)}(t) \quad$ Complex low-pass noise

$\mathbf{x}_{\mathrm{S}}[k] \quad$ Discrete MIMO non-frequency selective vector input

$\mathbf{x}_{\mathrm{s}}^{(1)}[k] \quad$ Discrete frequency selective input vector

$\mathbf{x}_{\mathbf{S}}^{(\nu)}[k] \quad$ Discrete MIMO frequency selective vector input

$x_{\mathrm{s}}^{(1)}[k] \quad$ Discrete baseband input signal

$x_{\mathrm{s}}^{(1)}(t) \quad$ SISO baseband input signal

$x_{\mathrm{s}}^{(\nu)}(t) \quad$ MIMO baseband input signal

$x_{\mathrm{s}, \mathrm{N}_{\mathrm{i}}}^{(1)}[k] \quad$ Discrete frequency selective input signal

$\mathbf{y}_{\mathrm{e}}[k] \quad$ Discrete MIMO non-frequency selective output vector

$\mathbf{y}_{\mathrm{e}}^{(1)}[k] \quad$ Discrete frequency selective output vector

$\mathbf{y}_{\mathrm{e}}^{(\mu)}[k] \quad$ Discrete MIMO frequency selective output vector 


\begin{tabular}{|c|c|}
\hline$y_{\mathrm{e}}^{(1)}[k]$ & Discrete baseband output signal \\
\hline$y_{\mathrm{e}}^{(1)}(t)$ & SISO baseband output signal \\
\hline$y_{\mathrm{e}}^{(\mu)}(t)$ & MIMO baseband output signal \\
\hline$y_{\mathrm{e}, \mathrm{N}_{\mathrm{o}}}^{(1)}[k]$ & Discrete frequency selective output signal \\
\hline$\delta(t)$ & Dirac delta function \\
\hline$\lambda$ & Wavelength \\
\hline$\lambda_{\mathrm{L}}$ & Lagrange multiplier \\
\hline$\nu$ & Variable \\
\hline$\mu$ & Variable \\
\hline$\hat{\mu}$ & Mean \\
\hline$\xi$ & Random scatter angle \\
\hline$\sqrt{\xi^{(\mu)}}$ & Singular value of $\mathbf{H}$ per layer \\
\hline$\rho$ & Antenna correlation coefficient \\
\hline$\rho_{\mathrm{RX}}^{(\mu \nu)}$ & Receive antennas correlation coefficient \\
\hline$\rho_{\mathrm{TX}}^{(\mu \nu)}$ & Transmit antennas correlation coefficient \\
\hline$\varrho$ & Signal noise rate \\
\hline$\varrho^{(\mu)}$ & Signal noise rate per layer \\
\hline$\sigma^{2}$ & Variance \\
\hline$\left(\sigma^{(\mu)}\right)^{2}$ & Noise power variance per layer \\
\hline$\sigma_{\xi}$ & Standard deviation of $\xi$ \\
\hline$\tau$ & Delay spread \\
\hline$\varphi$ & Spread angle \\
\hline$\varphi_{\mathrm{h}_{0}}$ & Phase complex Gaussian random variable \\
\hline
\end{tabular}




\section{Chapter 1}

\section{Introduction}

\subsection{State-of-the-art}

The use of multiple antennas is a key strategy to improve the performance of wireless systems [42]. Multiple-Input Multiple-Output (MIMO) systems have attracted significant attention since Foschini [18] and Telatar [35] analysed their potential to attain very high efficiency.

Nowadays, MIMO has become one of the scientific hot spot with thousands of research papers published around this topic during last years.

The strategy of placing multiple antennas at the transmitter and receiver side improves system performance due to the use of the spatial distribution characteristics of the wireless channel. Some of these improvements are related to the ability to increase the channel capacity, decrease the bit-error-rate (BER) without increasing the transmit power needed [11] and improve robustness and coverage through diversity combination [39].

The use of multiple transmit and receive antennas can be exploited to significantly increase channel capacity [21]. The capacity of MIMO systems increases linearly with the minimum number of antennas at both, the transmitter as well as the receiver side. The capacity improvement is highlighted in Figure 1-1 where a SISO system is compared with a $(4 \times 4)$ MIMO system.

Owing to these potential improvements in system performance and advances in 


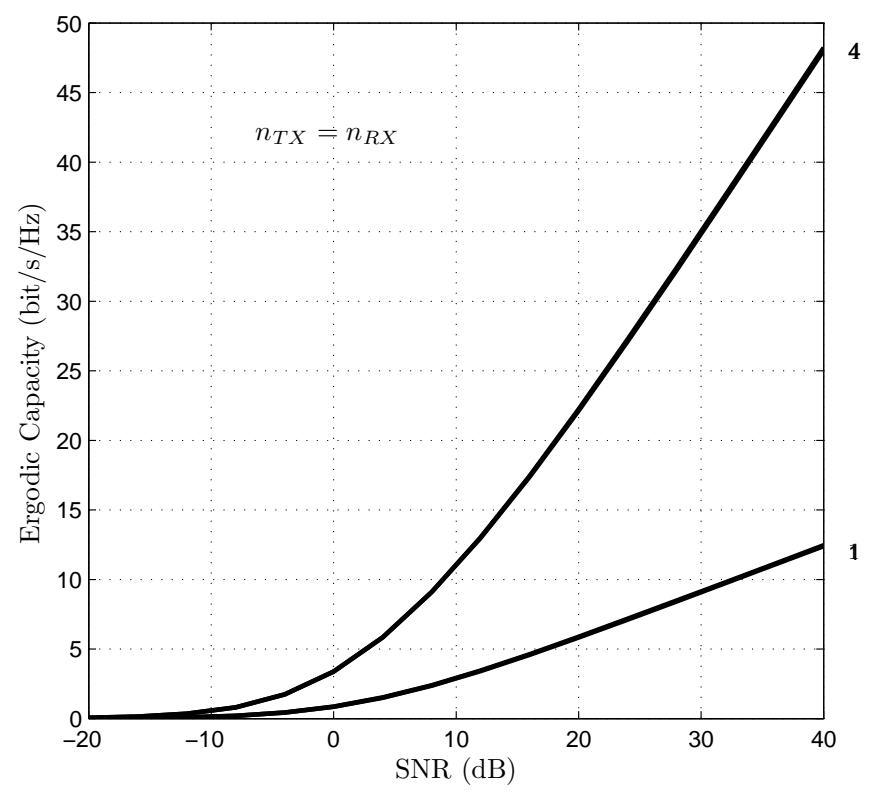

Figure 1-1: Capacity comparison between SISO $\left(n_{\mathrm{T}}=n_{\mathrm{R}}=1\right)$ and $\operatorname{MIMO}\left(n_{\mathrm{T}}=n_{\mathrm{R}}\right.$ $=4)$ frequency non-selective system

digital signal processing many wireless systems, including the IEEE 802.11n wireless LAN, IEEE 802.16e-based Mobile WiMAX ${ }^{\mathrm{TM}}$ Wave 2 and the Long-Term Evolution (LTE) mobile wireless system, have adopted the use of MIMO technology.

This doctoral thesis presents a brief description of some important theoretical concepts and models with the aim to properly define the wireless correlated MIMO communication systems.

Furthermore, proper signal processing techniques are required to eliminate the disable effects produced by MIMO techniques. In order to avoid inter-antenna interferences two mathematical decomposition techniques are used (Singular Value Decomposition and Geometric Mean Decomposition) to pre- and post-processing. These decomposition techniques transform a MIMO channel into multiple single-input singleoutput (SISO) channels, called layers [5].

However, the proximity between antennas introduces a phenomena called correlation. This effect produces interferences and decreases the quality of communication system [3]. MIMO scenarios with uncorrelated channel coefficients have reached a state of maturity. By contrast, MIMO scenarios with correlated channel coefficients 
require substantial further research. Antennas correlation diminishes multipath richness, essential to MIMO techniques. Due to that effect, the various paths from each transmitter-side antenna to each receiver-side antenna become similar. In this doctoral thesis the impact of the correlation between antennas over the MIMO channel performance has been characterized and investigated. Due to the results obtained the strong effect of the correlation over the quality criteria in MIMO systems is characterized and the huge dependence that correlation presents with the distance between antennas and the topology of the antennas' distribution.

Therefore, MIMO technology provides extra degrees of freedom which allows improving the performance of the MIMO communication link. Data rate, bits-per-layer and power allocation substantially affect the overall communication performance. Resource allocation techniques are aimed to minimize the overall bit-error rate.

Adaptive Modulation (AM) is a promising technique able to increase the spectral efficiency of wireless transmission systems by adapting the signal parameters, such as modulation constellation or transmit power, based on the uncertain channel conditions [43]. In order to achieve a better system performance given a fixed data rate an adaptive spatial modulation transmission scheme was proposed in [39].

The performance of MIMO systems using spatial multiplexing is analysed under bit- and power allocation techniques. Existing bit loading and transmit power allocation techniques are often optimized for maintaining both a fixed power and a fixed target bit-error rate while attempting to maximize the overall data-rate. However, delay-critical real-time interactive applications, such as voice or video transmission, may require a fixed data rate [4]. Provided perfect channel state information (PCSI) is available at the transmitter side two major optimization problems are considered to be solved. First, the optimal bit loading. Second, the power allocation optimization problem [37]. Given perfect channel state information at the transmitter, power and bits can be allocated to different layers. Adaptive bit and power allocation algorithms, which can significantly improve the MIMO system performance, have received a huge research activity lately, and can be divided into two groups according to their performance: optimal and suboptimal algorithms. Optimal allocation algo- 
rithms usually have high computational complexity, making them difficult to apply to practical communication systems [41].

In order to implement bit and power allocation in practical communication systems many computationally efficient suboptimal allocation algorithms have been proposed which most of them are iterative. Krongold proposed a Lagrange-multiplierbased integer-bits power allocation algorithm [27]. The algorithm projected by [20] minimizes the bit error rate $(\mathrm{BER})$ subject to a requested data rate and total transmit power by using adaptive power loading and uniform bit allocation over all subchannels. Zheng proposed a dynamic bound restriction iterative algorithm framework to reduce the computational complexity [41].

In this doctoral thesis an optimized scheme with fixed transmission modes per time slot is firstly analysed. Furthermore, the proposed algorithm performance has been compared to already developed strategies. The novel contribution of this research is the proposal of a new iterative bit and power allocation (IBPA) approach per MIMO layer based on unequal power distribution per MIMO active layer. The accomplished simulation results show the improvements made by these techniques over the BER MIMO curves reducing the bit error probability.

The single-user MIMO system model is extended by considering a single base station (BS) supporting $L_{\mathrm{MS}}$ mobile stations (MSs). Multi-user MIMO (MU-MIMO) systems refers to a link configuration comprising a base station with multiple $T_{\mathrm{x}}-R_{\mathrm{x}}$ antennas providing access to multiple users (fixed or mobile), each one equipped with multiple antennas (this doctoral thesis is focused on the downlink segment).

\subsection{Objectives}

The main goal of this doctoral thesis is to characterize the correlation effect over frequency non-selective $(4 \times 4)$ single-user MIMO systems performance under different

spatial antennas distribution (linear and non-linear) and analyse different resource allocation strategies in order to minimize the overall bit-error rate performance.

In this doctoral thesis the following problems are addressed: 
- Characterize the influence of the receiver-side antennas correlation over the BER in frequency non-selective $(4 \times 4)$ MIMO systems using the complementary cumulative distribution function (CCDF) over the singular values.

- Analyse different resources (number of active MIMO layers, number of the overall data rate, QAM modulation schema per layer and per time slot data rate, bits-per-layer and power) allocation techniques with the aim to increase the degree of design freedom, which largely affects the BER performance on correlated MIMO communication systems.

- Compare optimal and suboptimal Power Allocation (PA) techniques.

- Develop novel optimal resources allocation techniques based on iterative allocation methods over frequency non-selective $(4 \times 4)$ MIMO channels with and without antenna correlation.

- Compare the effect of both decomposition techniques, Singular Value Decomposition (SVD) and Geometric Mean Decomposition (GMD), over frequency non-selective $(4 \times 4)$ single-user MIMO Rayleigh channels in terms of BER performance.

- Extend the obtained results from the single-user MIMO (SU-MIMO) to the multi-user MIMO (MU-MIMO) and compare the BER performance.

- Eliminate multi-user and multi-antenna interferences using pre-and post-signal processing.

\section{Doctoral Thesis Organization}

This doctoral thesis is focused on the design and optimization of MIMO communications links with correlation. The transmission environment is specified to be a Rayleigh flat-fading channel with correlation at the transmitter side.

The doctoral thesis consists of 5 chapters. This first chapter presents a state-ofthe-art and the doctoral thesis chapters' structure. 
The second doctoral thesis chapter is focused on the MIMO channel. Chapter 2 includes two sections. Firstly, the wireless channel is described. A theoretical point of view and the impairments that this environment presents for a communication system like slow and fast fading are shown. It characterizes the Rayleigh statistical channel. In the second part of this first section, different existing communication models over the wireless digital channel are demonstrated. It models a SISO system for frequency non-selective channel and frequency selective channel (time and frequency domain and discrete model). These SISO models are extended to a $(4 \times 4)$ MIMO system. Secondly, the correlation concept and its impact in the reception antennas side in scattered environments are introduced. It has been also studied the incidence of two different antenna's distribution (linear and non-linear). Furthermore, the effect of the correlation parameters and the different distributions over a $(4 \times 4)$ MIMO communication link have been analysed. Finally, at the end of this second doctoral thesis chapter several CCDFs simulations related to the characterization of a $(4 \times 4)$ MIMO channel with and without correlation are included.

The third chapter of the doctoral thesis is focused on the signal processing over the MIMO system. Firstly, the mathematical singular value decomposition technique and its application in correlated MIMO systems are described. Moreover, the biterror rate concept and analysed in detail for MIMO systems is briefly introduced. Furthermore, a comparison between the BER curves with different QAM transmission modes in presence of correlation has been realized. Likewise, the power allocation concept with the optimal power allocation technique based on the Lagrange multiplier method, the suboptimal equal signal-to-noise ratio (SNR) power allocation, the waterfilling techniques and the Iterative Bit and Power Allocation (IBPA) algorithm are introduced. The aim of the power allocation techniques is to achieve better BER performance in frequency non-selective MIMO communication systems. Different simulations have been accomplished in order to show the remarkable improvement of different power allocation techniques over correlated MIMO communication links. Finally, a comparison between SVD and GMD techniques is accomplished.

The fourth chapter of the doctoral thesis is focused on the multi-user MIMO 
communication systems where the system model consists of a single base station (BS) supporting $L_{\mathrm{MS}}$ mobile stations (MSs). Also, the required pre-and post-processing in order to be able to perfect eliminate the multi-user and multi-antenna interferences is analysed.

In the final fifth chapter, the most relevant concluding remarks of this doctoral thesis are presented and some possible trends of further research are proposed. Finally, the list of publications is demonstrated. 


\section{Chapter 2}

\section{MIMO Channel}

Wireless MIMO channels have been attracting great research efforts as the great number of publications released along last years [19], [32], [22] [6]. MIMO techniques enable the improvement of communication performance by increasing the channel reliability with respect to SISO channels [23].

MIMO channel corresponds with a signal propagation through a wireless channel which arrives at the destination along a number of different paths, referred to as multipath. These paths arise from different physical phenomena like scattering, reflection and diffraction of the radiated energy by objects in the environment or refraction in the medium. These phenomena produce path loss and fading.

\subsubsection{Wireless Radio Channel}

The performance of wireless communication system is mainly governed by the wireless channel environment. As opposed to the typical static and predictable characteristics of a wired channel, the wireless channel is rather than dynamic and unpredictable, which makes an exact analysis of the wireless communication system often difficult [15].

In recent years, critical optimization of the wireless communication system has emerged as wireless communication channel has become one of the most important communication channels in the last years due to the proliferation of handset and 
mobile devices and the growing reliability of the wireless communication.

The wireless channel presents by nature. However, several limitations has to be solved. The most typical impairments of the wireless channel in Line-Of-Sight (LOS) environments are: inter-symbol interference, noise, co-channel interference, path-losses and scarce available bandwidth.

Furthermore, there are Non-Line-of-Sight (NLOS) scenarios which are the most common due to the emerging broadband mobile Internet access services. The increasing wireless communication services requires most of them in-home environments where it is common to have several wireless devices working at the same time for different functions but requiring all of them to be interoperable.

During the trajectory of radio propagation waves in wireless communication, radio waves can be corrupted due to the changeable environment. As a consequence, mobile radio transmission usually suffers large fluctuations in the level signal like attenuation, interferences or time delay.

The most significant physical phenomena that affect signal propagation in a mobile wireless communication system are as following: Reflection, Diffraction and Scattering.

Reflection. It is the physical phenomena that occur when a propagating electromagnetic waves impact over an object much larger in comparison with the wavelength, for example, surface of the oceans or mountains. It produces a change in the direction of the transmitted signal waves going back the path towards to their origin.

Diffraction. Refers to the physical phenomena that occur when the electromagnetic waves find a sharp surface or small openings in the path between the transmitter and receiver. It appears as a bending of waves generated by diffraction after crossing the openings. Those produced diffraction waves are useful for establishing a path between the transmitter and the receiver, even when a LOS path is not available.

Scattering. It is the physical phenomena that force the radiation of an electromagnetic wave to diverge from a straight path by one or more local obstacles, with 
small dimensions compared to the wavelength. Those obstacles such as city-furniture, vehicles or trees that generate interferences or scattering are called scatters.

In resume, the propagation of a radio wave depends on reflection, diffraction and scattering, which modify the signal making the process less predictable and more subordinated to the environment.

A unique characteristic in a wireless channel is a phenomena called 'fading', which consists of important changes and variations in the signal amplitude over time and frequency.

Fading denotes a source of signal degradation characterized as a non-additive signal disturbance in the wireless channel. Fading is one of the most difficult impairments to avoid due to multipath propagation, referred as multipath fading or shadow fading.

The fading phenomena can be classified in two groups:

Large-scale Fading. Occurs due to the movements from a mobile receptor along large distances, for example, distances of the order of cell sizes. It is caused by path loss of signal as a function of distance and shadowing is produced by large objects such as buildings. Shadowing is a slow fading process characterized by variation of median path loss between the transmitter and receiver in fixed locations. In resume, large-scale fading is characterized by average path loss and shadowing.

Small-scale Fading. Refers to fast variations of signal levels due to the constructive and destructive interference of multiple signal paths (multipath) when the mobile station makes short and fast movements. Multipath is originated in scattered environments when multiple waves from the transmitter arrive in the receiver with a delay and others different modifications (for example, phase shift) due to the collision of the signal with objects that the waves find in its path towards the receiver. Depending on the relative extent of a multipath, frequency selectivity of a channel is characterized between frequency-selective channel or frequency non-selective channel. 


\section{Slow Fading}

Small-scale multipath fading is more relevant to the design of reliable and efficient communication systems [36].

Small-scale fading is referred to fading in short. Fading is the rapid variation of the received signal level in the short term as the user terminal moves a short distance. It is due to the effect of multiple signal paths, which cause interference when they arrive subsequently in the receive antenna with varying phases (i.e constructive interferences with the same phase and destructive interference with the opposite phase). In other words, the variation of the received signal level depends on the relationship between the relative phases among the number of signals reflected from the local scatters. Furthermore, each of the multiple signal paths may undergo changes that depend on the speed of the mobile stations and surrounding objects. In summary, small-scale fading is attributed to multipath propagation, mobile speed, speed of surrounding objects and transmission bandwidth of signal.

Time Dispersion. Due to time dispersion, a transmit signal may undergo fading over a frequency domain either in a selective or non-selective manner, which is referred to as frequency-selective fading or frequency non-selective fading, respectively. For the given channel frequency response, frequency selectivity is generally governed by signal bandwidth [15].

On the one hand, the transmitted signal is subject to frequency non-selective fading when signal bandwidth is narrow enough that it may be transmitted over the flat response. Because of that the symbol period $T_{\mathrm{s}}$ is greater than delay spread $\tau$ of the multipath channel. As long as $T_{\mathrm{s}}$ is greater than $\tau$, the current symbol does not affect the subsequent symbol as much over the next symbol period, implying that inter-symbol interference (ISI) is not significant.

On the other hand, the signal is subject to frequency-selective fading when signal bandwidth is wide enough that it may be filtered out by the finite channel bandwidth. Hence, due to $\tau$ that is greater than $T_{\mathrm{s}}$, multiple-delayed copies of the transmit signal are significantly overlapped with the subsequent symbol, incurring inter-symbol 
interference.

\section{Statistical Models for Frequency Non-Selective Channels}

There are several probability distributions that can be considered in attempting to model the statistical characteristics of the fading channel [33]. For a large number of propagation paths, real and imaginary parts of the channel are statistically independent and Gaussian distributed stochastic processes [28]. The whole channel is Rayleigh distributed and is defined by

$$
g_{\mathrm{k}}^{(11)}(t)=h_{0} \cdot \delta(t)
$$

where $g_{\mathrm{k}}^{(11)}(t)$ is the impulse response, $\delta(t)$ is the Dirac delta function and $h_{0}$ is a complex Gaussian random variable with zero mean and variance $\sigma^{2}=E\left\{\left|h_{0}\right|\right\}$. The phase $\varphi_{h_{0}}$ is the uniform over the range $(0,2 \pi)$ and independent of the magnitude $\left\|h_{0}\right\|$. The probability density function is given by

$$
p_{\left|h_{0}\right|}(r)=\left\{\begin{array}{l}
\frac{2 r}{\sigma^{2}} \cdot e^{\frac{-r^{2}}{\sigma^{2}}} \text { if } r \geq 0 \\
0 \text { otherwise }
\end{array}\right.
$$

where $r$ represents the amplitude of the probability density function.

\subsubsection{Model of the Wireless Channel}

\section{SISO Frequency Non-selective Channel}

In Figure 2-1 the time model of the frequency non-selective channel is observed. According to this figure, the SISO frequency non-selective Rayleigh channel link is characterized by the equation (2.3)

$$
u_{\mathrm{e}}^{(1)}(t)=u_{\mathrm{k}}^{(1)}(t)+w^{(1)}(t)
$$

where $u_{\mathrm{k}}^{(1)}(t)$ is the signal transmitted through the channel, $w^{(1)}(t)$ the noise in the receiver side and $u_{\mathrm{e}}^{(1)}(t)$ the received signal. Figure 2-1 shows the parameters 


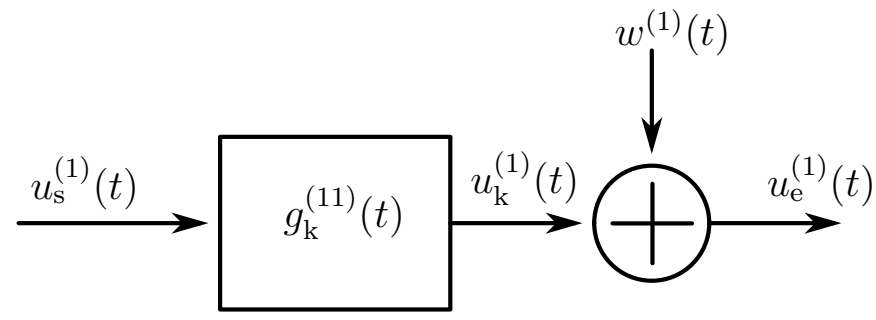

Figure 2-1: Time model of SISO frequency non-selective channel

$u_{\mathrm{s}}^{(1)}(t)$, defined as the input signal, and $g_{\mathrm{k}}^{(11)}(t)$, which represents the Rayleigh channel descriptor defined in the equation (2.1)

$$
u_{\mathrm{k}}^{(1)}(t)=u_{\mathrm{s}}^{(1)}(t) * g_{\mathrm{k}}^{(11)}(t)
$$

According to the equations (2.3) and (2.4), it is developed the well-known Rayleigh link expression denoted by

$$
u_{\mathrm{e}}^{(1)}(t)=u_{\mathrm{s}}^{(1)}(t) * g_{\mathrm{k}}^{(11)}(t)+w^{(1)}(t)
$$

\section{SISO Frequency Selective Channel}

The impulse response for frequency selective channel is

$$
g_{\mathrm{k}}^{(11)}(t)=\sum_{\nu=0}^{q_{\mathrm{k}}-1} g_{\mathrm{k} \nu}^{(11)}(t) \cdot \delta\left(t-\tau_{\nu}\right)
$$

where $g_{\mathrm{k} \nu}^{(11)}(t)$ with $\nu=1, \ldots, L$ defines the number of delay taps from 1 to $L$. This expression takes into account the effect produced for the numerous scatters which delay the $\nu$-paths that are transmitted at time $t$ and arrive at time $t-\tau_{\nu}$.

In Figure 2-2 a system model is represented which is commonly used to characterize the frequency-selective channels. The signal is passed through a tapped-delay-line and weighted at each tap with complex channel coefficients $g_{\mathrm{k} \nu}^{(11)}(t)$. In Figure 2-2 one delay tap $\tau_{1}$ appears, and in consequence the effect of two channel coefficients $g_{\mathrm{k} 0}^{(11)}(t)$ and $g_{\mathrm{k} 1}^{(11)}(t)$ is considered. 


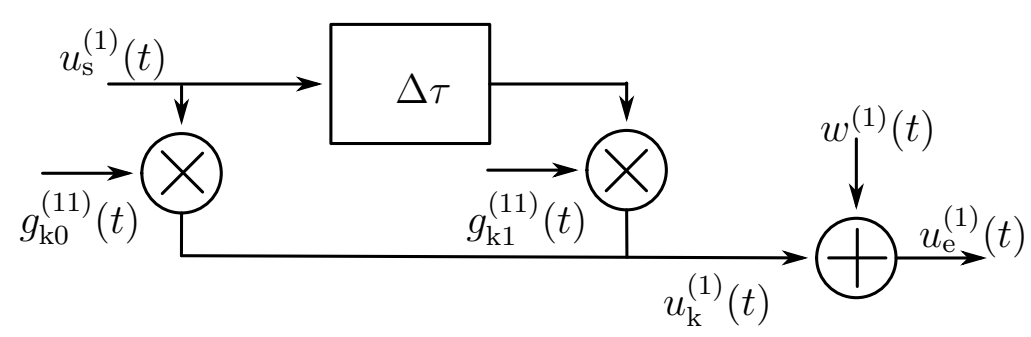

Figure 2-2: Time model of SISO frequency selective channel

Frequency Domain. A frequency selective channel in the frequency domain is characterized by the following development from the time signal $g_{\mathrm{k}}^{(11)}(t)$

$$
g_{\mathrm{k}}^{(11)}(t)=g_{\mathrm{k} 0}^{(11)}(t) \cdot \delta\left(t-\tau_{1}\right)+g_{\mathrm{k} 1}^{(11)}(t) \cdot \delta\left(t-\tau_{2}\right)
$$

Assuming that $g_{\mathrm{k} 0}^{(11)}(t)=g_{\mathrm{k} 1}^{(11)}(t)=1$

$$
g_{\mathrm{k}}^{(11)}(t)=\delta(t)+\delta(t-\Delta \tau),
$$

where $\Delta \tau=\tau_{2}-\tau_{1}$.

Applying Fourier transform, the obtained equation is

$$
G_{\mathrm{k}}^{(11)}(f)=1+e^{-j 2 \pi f \Delta \tau}
$$

The module of the frequency selective channel in the frequency domain is obtained by

$$
\left|G_{\mathrm{k}}^{(11)}(f)\right|=\sqrt{(1+\cos (2 \pi f \Delta \tau))^{2}+(\sin (2 \pi f \Delta \tau))^{2}}=\sqrt{2+2 \cos (2 \pi f \Delta \tau)}
$$

where the simplified expression results in

$$
\left|G_{\mathrm{k}}^{(11)}(f)\right|=2 \cdot|\cos (\pi f \Delta \tau)|
$$

\section{SISO Baseband Equivalent Model}

Figure 2-3 shows a baseband equivalent model of a SISO communication system. 


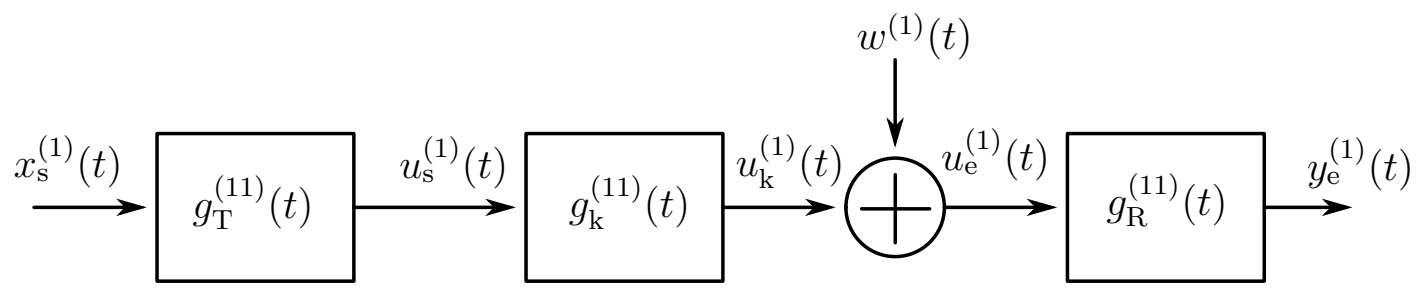

Figure 2-3: Time model of SISO baseband equivalent model

From this figure, the following equations which characterize a SISO Baseband Equivalent Model are obtained

$$
\begin{aligned}
& y_{\mathrm{e}}^{(1)}(t)=u_{\mathrm{e}}^{(1)}(t) * g_{\mathrm{R}}^{(11)}(t) . \\
& u_{\mathrm{e}}^{(1)}(t)=u_{\mathrm{k}}^{(1)}(t)+w^{(1)}(t) . \\
& u_{\mathrm{k}}^{(1)}(t)=u_{\mathrm{s}}^{(1)}(t) * g_{\mathrm{k}}^{(11)}(t) . \\
& u_{\mathrm{s}}^{(1)}(t)=x_{\mathrm{s}}^{(1)}(t) * g_{\mathrm{T}}(t) .
\end{aligned}
$$

According to the previous equations, it is developed the SISO baseband expression denoted by

$$
y_{\mathrm{e}}^{(1)}(t)=\left(g_{\mathrm{T}}(t) * x_{\mathrm{s}}^{(1)}(t) * g_{\mathrm{k}}^{(11)}(t)+w^{(1)}(t)\right) * g_{\mathrm{R}}^{(11)}(t)
$$

The input signal $x_{\mathrm{s}}^{(1)}(t)$ is defined by the equation

$$
x_{\mathrm{s}}^{(1)}(t)=T_{\mathrm{s}} \sum_{k=-\infty}^{+\infty} a[k] \cdot \delta\left(t-n T_{\mathrm{s}}\right)
$$

where $T_{\mathrm{s}}$ is the symbol rate and $a[k]$ means the modulated transmit data.

In Figure 2-4 the baseband equivalent model after the convolution of the transmit and receiver filters and the channel response based on the following expression are 


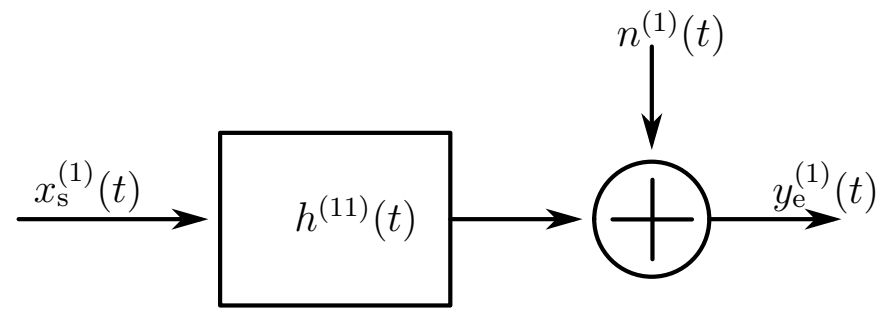

Figure 2-4: Time model of SISO baseband equivalent model simplified

shown

$$
g_{\mathrm{T}}(t) * g_{\mathrm{k}}^{(11)}(t) * g_{\mathrm{R}}(t)=h^{(11)}(t)
$$

Finally, the relationship between the input and the output in a SISO baseband model is obtained

$$
y_{\mathrm{e}}^{(1)}(t)=h^{(11)}(t) * x_{\mathrm{s}}^{(1)}(t)+n^{(1)}(t)
$$

where $n^{(1)}(t)$ is the noise convolution product between the receiver filter and the complex low-pass noise, $n^{(1)}(t)=g_{\mathrm{R}}(t) * w^{(1)}(t)$.

Discrete Model of SISO Frequency Non-selective Channel. Figure 2-5 describes the following input-output vector-matrix SISO frequency non-selective system model.

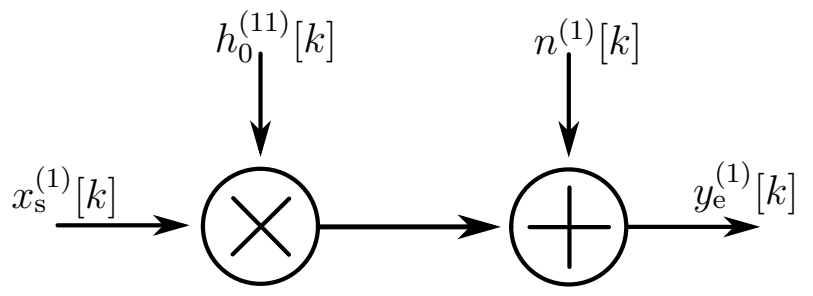

Figure 2-5: Discrete model of SISO frequency non-selective channel

$$
y_{\mathrm{e}}^{(1)}[k]=h_{0}^{(11)}[k] \cdot x_{\mathrm{s}}^{(1)}[k]+n^{(1)}[k] .
$$


Discrete Model of SISO Frequency Selective Channel. In Figure 2-6 the input-output vector-matrix SISO frequency selective system model is described.

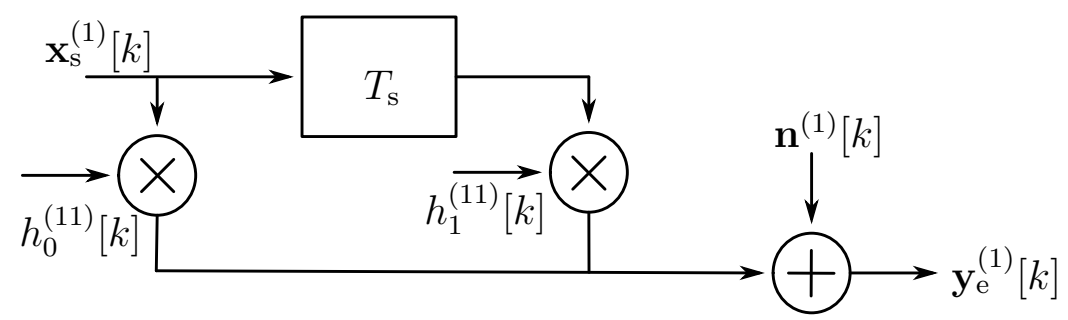

Figure 2-6: Discrete model of SISO frequency selective channel

$$
\begin{aligned}
& \left(\begin{array}{c}
y_{\mathrm{e}, 1}^{(1)}[k] \\
y_{\mathrm{e}, 2}^{(1)}[k] \\
\vdots \\
y_{\mathrm{e}, \mathrm{N}_{\mathrm{o}}}^{(1)}[k]
\end{array}\right)=\left(\begin{array}{cccc}
h_{0}^{(11)}[k] & 0 & \cdots & 0 \\
h_{1}^{(11)}[k] & h_{0}^{(11)}[k] & \cdots & 0 \\
\vdots & \vdots & \ddots & \vdots \\
0 & 0 & 0 & h_{\mathrm{L}}^{(11)}[k]
\end{array}\right) \cdot\left(\begin{array}{c}
x_{\mathrm{s}, 1}^{(1)}[k] \\
x_{\mathrm{s}, 2}^{(1)}[k] \\
\vdots \\
x_{\mathrm{s}, \mathrm{N}_{\mathrm{i}}}^{(1)}[k]
\end{array}\right)+\left(\begin{array}{c}
n_{1}^{(1)}[k] \\
n_{2}^{(1)}[k] \\
\vdots \\
n_{\mathrm{N}_{\mathrm{o}}}^{(1)}[k]
\end{array}\right) \\
& \mathbf{y}_{\mathrm{e}}^{(1)}[k]=\mathbf{H}^{(11)}[k] \cdot \mathbf{x}_{\mathrm{s}}^{(1)}[k]+\mathbf{n}^{(1)}[k] .
\end{aligned}
$$

\section{MIMO Baseband Equivalent Model}

Figure 2-7 illustrates a general MIMO system model where it is possible to appreciate the interferences produced in the outputs by the inputs.

Taking into account the signal input $u_{\mathrm{s}}^{(1)}(t)$

$$
u_{\mathrm{s}}^{(1)}(t)=x_{\mathrm{s}}^{(1)}(t) * g_{\mathrm{T}}(t)
$$

the resulting signal is multiplied by the Rayleigh channel parameters

$$
u_{\mathrm{k}}^{(11)}(t)=u_{\mathrm{s}}^{(1)}(t) * g_{\mathrm{k}}^{(11)}(t) .
$$




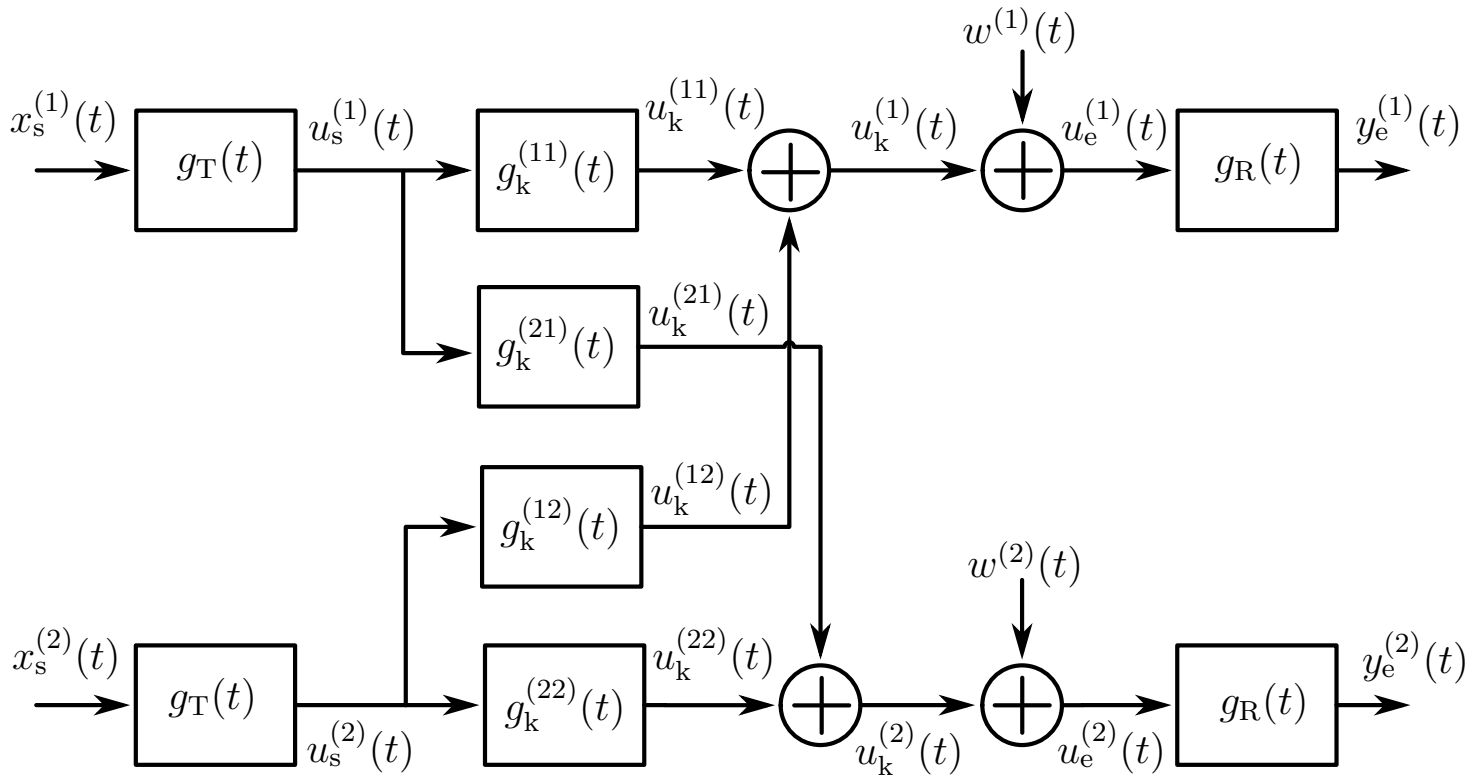

Figure 2-7: Time model of MIMO frequency non-selective channel

$$
u_{\mathrm{k}}^{(12)}(t)=u_{\mathrm{s}}^{(2)}(t) * g_{\mathrm{k}}^{(12)}(t)
$$

Moreover, the effects produced by the interference over the first output signal in the Rayleigh channel parameters from the second signal input are added

$$
u_{\mathrm{k}}^{(1)}(t)=u_{\mathrm{k}}^{(11)}(t)+u_{\mathrm{k}}^{(12)}(t),
$$

as well as the signal noise is considered

$$
u_{\mathrm{e}}^{(1)}(t)=u_{\mathrm{k}}^{(1)}(t)+w^{(1)}(t)
$$

Finally, the output signal in Layer 1 is obtained

$$
y_{\mathrm{e}}^{(1)}(t)=u_{\mathrm{e}}^{(1)}(t) * g_{\mathrm{R}}(t)
$$

On the other hand, the second signal input $u_{\mathrm{s}}^{(2)}(t)$ is taken into consideration

$$
u_{\mathrm{s}}^{(2)}(t)=x_{\mathrm{s}}^{(2)}(t) * g_{\mathrm{T}}(t)
$$

The resulting signal is multiplied by the Rayleigh channel parameters 


$$
\begin{aligned}
& u_{\mathrm{k}}^{(21)}(t)=u_{\mathrm{s}}^{(1)}(t) * g_{\mathrm{k}}^{(21)}(t) . \\
& u_{\mathrm{k}}^{(22)}(t)=u_{\mathrm{s}}^{(2)}(t) * g_{\mathrm{k}}^{(22)}(t) .
\end{aligned}
$$

Afterwards the effects produced by the interference over the second output signal in the Rayleigh channel parameters from the first signal input are added

$$
u_{\mathrm{k}}^{(2)}(t)=u_{\mathrm{k}}^{(21)}(t)+u_{\mathrm{k}}^{(22)}(t) .
$$

Later on, the signal noise $w^{(2)}(t)$ is introduced

$$
u_{\mathrm{e}}^{(2)}(t)=u_{\mathrm{k}}^{(2)}(t)+w^{(2)}(t) .
$$

To conclude, the output signal in Layer 2 results

$$
y_{\mathrm{e}}^{(2)}(t)=u_{\mathrm{e}}^{(2)}(t) * g_{\mathrm{R}}(t) .
$$

Taking into consideration the convolution of the transmit-receiver filters and the channel,

$$
g_{\mathrm{T}}(t) * g_{\mathrm{k}}^{(\mu \nu)}(t) * g_{\mathrm{R}}(t)=h^{(\mu \nu)}(t) .
$$

The overall relationship between input and output signal in time domain of MIMO communication system is described by

$$
y_{\mathrm{e}}^{(\mu)}(t)=\sum_{\nu=1}^{n_{\mathrm{T}}} h^{(\mu \nu)}(t) * x_{\mathrm{s}}^{(\nu)}(t)+n^{(\mu)}(t),
$$

with $\mu=1, \ldots, n_{\mathrm{R}}$.

Discrete Model of MIMO Frequency Non-selective Channel. In Figure 2-8 the discrete model of the MIMO frequency non-selective channel is depicted. From the figure, the following expressions are obtained 


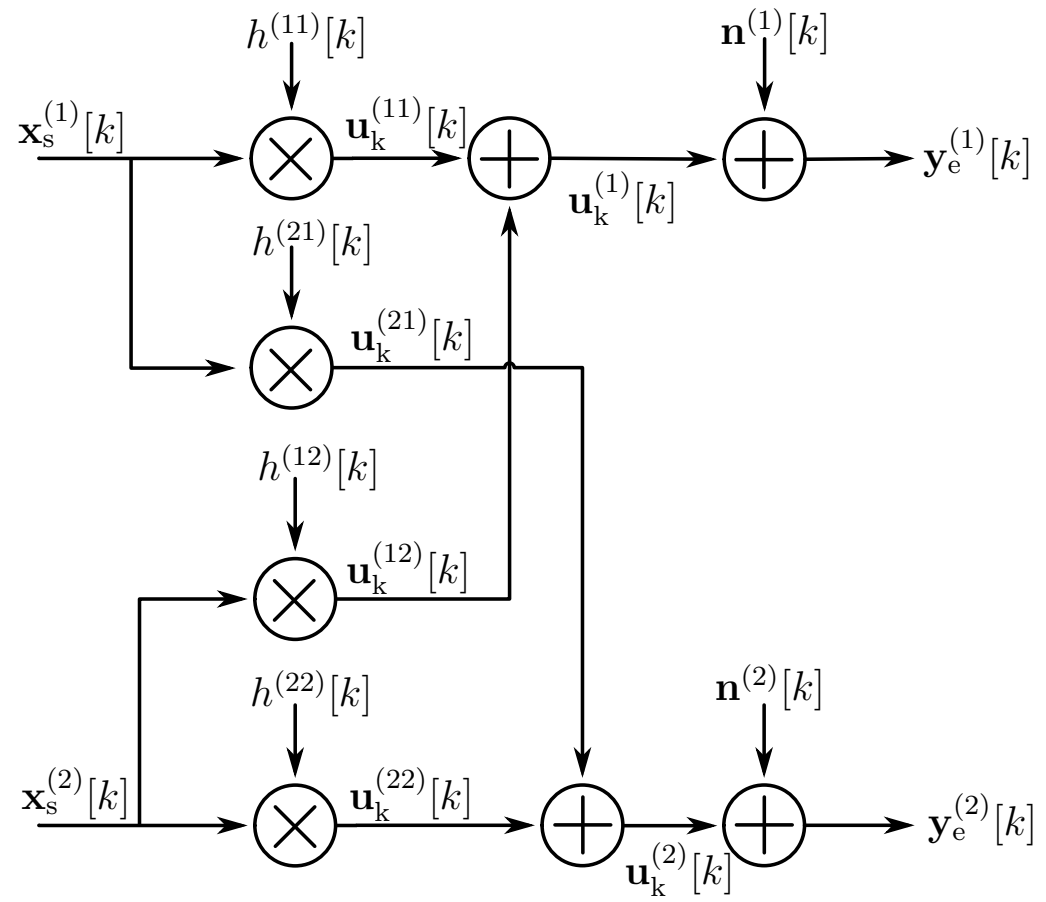

Figure 2-8: Discrete model of MIMO frequency non-selective channel

$$
\begin{aligned}
& y_{\mathrm{e}}^{(1)}[k]=u_{\mathrm{k}}^{(1)}[k]+n^{(1)}[k] . \\
& u_{\mathrm{k}}^{(1)}[k]=u_{\mathrm{k}}^{(11)}[k]+u_{\mathrm{k}}^{(12)}[k] . \\
& u_{\mathrm{k}}^{(11)}[k]=h^{(11)}[k] \cdot x_{\mathrm{s}}^{(1)}[k] . \\
& u_{\mathrm{k}}^{(12)}[k]=h^{(12)}[k] \cdot x_{\mathrm{s}}^{(2)}[k] . \\
& u_{\mathrm{k}}^{(2)}[k]=u_{\mathrm{k}}^{(21)}[k]+u_{\mathrm{k}}^{(22)}[k] . \\
& u_{\mathrm{k}}^{(21)}[k]=h^{(21)}[k] \cdot x_{\mathrm{s}}^{(1)}[k] .
\end{aligned}
$$




$$
u_{\mathrm{k}}^{(22)}[k]=h^{(22)}[k] \cdot x_{\mathrm{s}}^{(2)}[k] .
$$

Analysis of all previous formulas allows developing the vector-matrix system model by the following expression

$$
\left(\begin{array}{l}
y_{\mathrm{e}}^{(1)}[k] \\
y_{\mathrm{e}}^{(2)}[k]
\end{array}\right)=\left(\begin{array}{ll}
h^{(11)}[k] & h^{(12)}[k] \\
h^{(21)}[k] & h^{(22)}[k]
\end{array}\right) \cdot\left(\begin{array}{c}
x_{\mathrm{s}}^{(1)}[k] \\
x_{\mathrm{s}}^{(2)}[k]
\end{array}\right)+\left(\begin{array}{c}
n^{(1)}[k] \\
n^{(2)}[k]
\end{array}\right)
$$

with the simplified form

$$
\mathbf{y}_{\mathrm{e}}[k]=\mathbf{H}[k] \cdot \mathbf{x}_{\mathrm{s}}[k]+\mathbf{n}[k]
$$

Discrete Model of MIMO Frequency Selective Channel. In Figure 2-9 the discrete model of the MIMO frequency selective channel with one delay tap is analysed. Examination of this figure allows describing the following expressions

$$
\begin{aligned}
& \mathbf{y}_{\mathrm{e}}^{(1)}[k]=\mathbf{u}_{\mathrm{k}}^{(1)}[k]+\mathbf{n}^{(1)}[k] \\
& \mathbf{u}_{\mathrm{k}}^{(1)}[k]=\mathbf{u}_{\mathrm{k}}^{(11)}[k]+\mathbf{u}_{\mathrm{k}}^{(12)}[k] \\
& \mathbf{u}_{\mathrm{k}}^{(11)}[k]=\mathbf{H}^{(11)}[k] \cdot \mathbf{x}_{\mathrm{s}}^{(1)}[k]
\end{aligned}
$$

where

$$
\mathbf{H}^{(11)}[k]=\left(\begin{array}{cccc}
h_{0}^{(11)}[k] & 0 & \cdots & 0 \\
h_{1}^{(11)}[k] & h_{0}^{(11)}[k] & \cdots & 0 \\
\vdots & \vdots & \ddots & \vdots \\
0 & 0 & 0 & h_{\mathrm{L}}^{(11)}[k]
\end{array}\right) .
$$




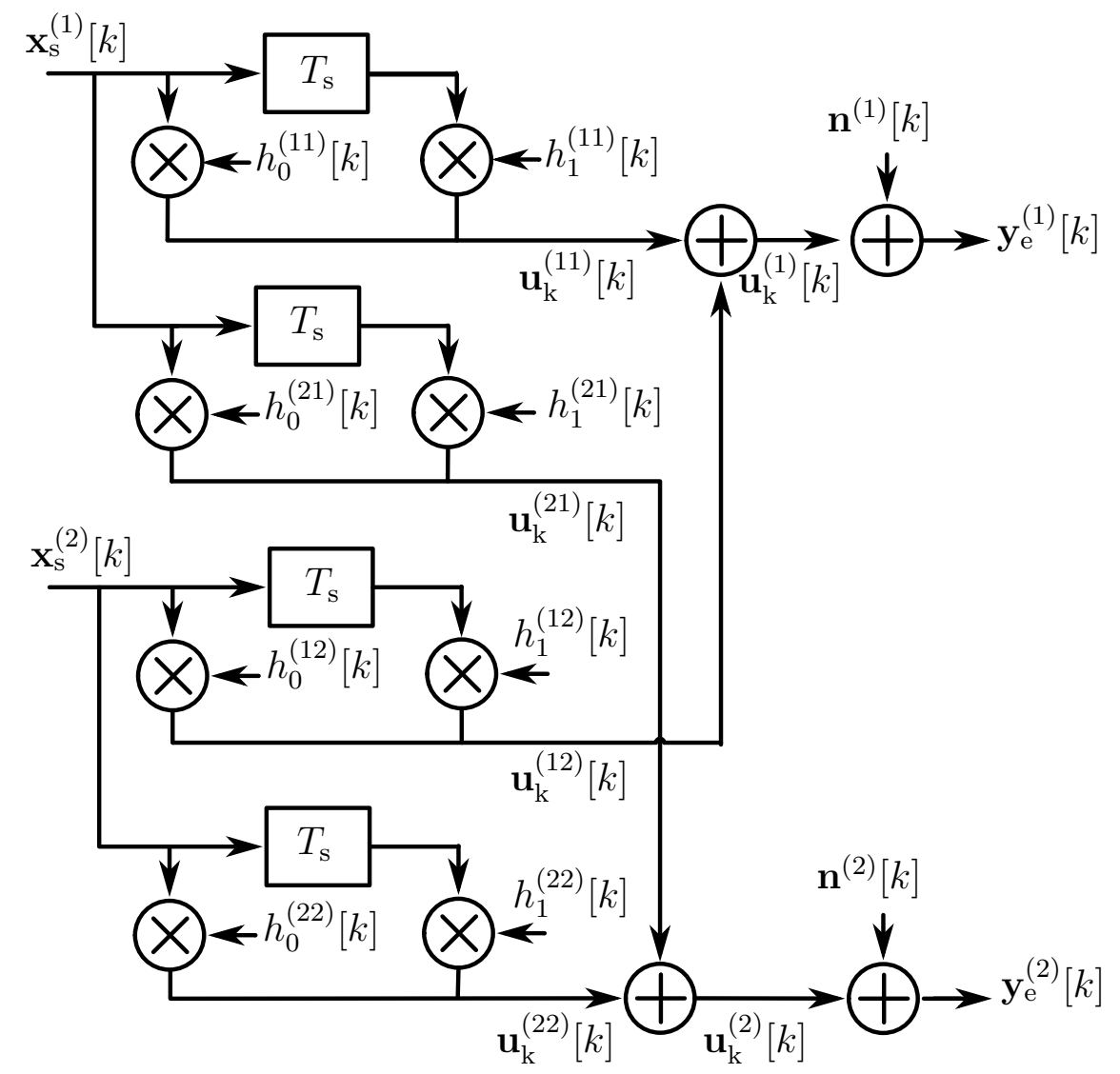

Figure 2-9: Discrete model of MIMO frequency selective channel 


$$
\mathbf{u}_{\mathrm{k}}^{(12)}[k]=\mathbf{H}^{(12)}[k] \cdot \mathbf{x}_{\mathrm{s}}^{(2)}[k]
$$

where

$$
\begin{gathered}
\mathbf{H}^{(12)}[k]=\left(\begin{array}{cccc}
h_{0}^{(12)}[k] & 0 & \cdots & 0 \\
h_{1}^{(12)}[k] & h_{0}^{(11)}[k] & \cdots & 0 \\
\vdots & \vdots & \ddots & \vdots \\
0 & 0 & 0 & h_{\mathrm{L}}^{(12)}[k]
\end{array}\right) \\
\mathbf{u}_{\mathrm{k}}^{(2)}[k]=\mathbf{u}_{\mathrm{k}}^{(21)}[k]+\mathbf{u}_{\mathrm{k}}^{(22)}[k] \\
\mathbf{u}_{\mathrm{k}}^{(21)}[k]=\mathbf{H}^{(21)}[k] \cdot \mathbf{x}_{\mathrm{s}}^{(1)}[k]
\end{gathered}
$$

where

$$
\begin{gathered}
\mathbf{H}^{(21)}[k]=\left(\begin{array}{cccc}
h_{0}^{(21)}[k] & 0 & \cdots & 0 \\
h_{1}^{(21)}[k] & h_{0}^{(21)}[k] & \cdots & 0 \\
\vdots & \vdots & \ddots & \vdots \\
0 & 0 & 0 & h_{\mathrm{L}}^{(21)}[k]
\end{array}\right) \\
\mathbf{u}_{\mathrm{k}}^{(22)}[k]=\mathbf{H}^{(22)}[k] \cdot \mathbf{x}_{\mathrm{s}}^{(2)}[k]
\end{gathered}
$$

where

$$
\mathbf{H}^{(22)}[k]=\left(\begin{array}{cccc}
h_{0}^{(22)}[k] & 0 & \cdots & 0 \\
h_{1}^{(22)}[k] & h_{0}^{(22)}[k] & \cdots & 0 \\
\vdots & \vdots & \ddots & \vdots \\
0 & 0 & 0 & h_{\mathrm{L}}^{(22)}[k]
\end{array}\right)
$$

The vector-matrix system model is developed by the following formulas 


$$
\begin{aligned}
& \left(\begin{array}{c}
y_{\mathrm{e}, 1}^{(1)}[k] \\
y_{\mathrm{e}, 2}^{(1)}[k] \\
\vdots \\
y_{\mathrm{e}, \mathrm{N}_{\mathrm{o}}}^{(1)}[k]
\end{array}\right)=\left(\begin{array}{cccc}
h_{0}^{(11)}[k] & 0 & \cdots & 0 \\
h_{1}^{(11)}[k] & h_{0}^{(11)}[k] & \cdots & 0 \\
\vdots & \vdots & \ddots & \vdots \\
0 & 0 & 0 & h_{\mathrm{L}}^{(11)}[k]
\end{array}\right) \cdot\left(\begin{array}{c}
x_{\mathrm{s}, 1}^{(1)}[k] \\
x_{\mathrm{s}, 2}^{(1)}[k] \\
\vdots \\
x_{\mathrm{s}, \mathrm{N}_{\mathrm{i}}}^{(1)}[k]
\end{array}\right)+ \\
& +\left(\begin{array}{cccc}
h_{0}^{(12)}[k] & 0 & \cdots & 0 \\
h_{1}^{(12)}[k] & h_{0}^{(12)}[k] & \cdots & 0 \\
\vdots & \vdots & \ddots & \vdots \\
0 & 0 & 0 & h_{\mathrm{L}}^{(12)}[k]
\end{array}\right) \cdot\left(\begin{array}{c}
x_{\mathrm{s}, 1}^{(2)}[k] \\
x_{\mathrm{s}, 2}^{(2)}[k] \\
\vdots \\
x_{\mathrm{s}, \mathrm{N}_{\mathrm{i}}}^{(2)}[k]
\end{array}\right)+\left(\begin{array}{c}
n_{1}^{(1)}[k] \\
n_{2}^{(1)}[k] \\
\vdots \\
n_{\mathrm{N}_{\mathrm{o}}}^{(1)}[k]
\end{array}\right)
\end{aligned}
$$

and

$$
\begin{aligned}
& \left(\begin{array}{c}
y_{\mathrm{e}, 1}^{(2)}[k] \\
y_{\mathrm{e}, 2}^{(2)}[k] \\
\vdots \\
y_{\mathrm{e}, \mathrm{N}_{\mathrm{o}}}^{(2)}[k]
\end{array}\right)=\left(\begin{array}{cccc}
h_{0}^{(21)}[k] & 0 & \cdots & 0 \\
h_{1}^{(21)}[k] & h_{0}^{(21)}[k] & \cdots & 0 \\
\vdots & \vdots & \ddots & \vdots \\
0 & 0 & 0 & h_{\mathrm{L}}^{(21)}[k]
\end{array}\right) \cdot\left(\begin{array}{c}
x_{\mathrm{s}, 1}^{(1)}[k] \\
x_{\mathrm{s}, 2}^{(1)}[k] \\
\vdots \\
x_{\mathrm{s}, \mathrm{N}_{\mathrm{i}}}^{(1)}[k]
\end{array}\right)+ \\
& +\left(\begin{array}{cccc}
h_{0}^{(22)}[k] & 0 & \cdots & 0 \\
h_{1}^{(22)}[k] & h_{0}^{(22)}[k] & \cdots & 0 \\
\vdots & \vdots & \ddots & \vdots \\
0 & 0 & 0 & h_{\mathrm{L}}^{(22)}[k]
\end{array}\right) \cdot\left(\begin{array}{c}
x_{\mathrm{s}, 1}^{(2)}[k] \\
x_{\mathrm{s}, 2}^{(2)}[k] \\
\vdots \\
x_{\mathrm{s}, \mathrm{N}_{\mathrm{i}}}^{(2)}[k]
\end{array}\right)+\left(\begin{array}{c}
n_{1}^{(2)}[k] \\
n_{2}^{(2)}[k] \\
\vdots \\
n_{\mathrm{N}_{\mathrm{o}}}^{(2)}[k]
\end{array}\right) .
\end{aligned}
$$

Equations (2.57) and (2.58) can be jointly expressed by a general matrix relationship

$$
\left(\begin{array}{l}
\mathbf{y}_{\mathrm{e}}^{(1)}[k] \\
\mathbf{y}_{\mathrm{e}}^{(2)}[k]
\end{array}\right)=\left(\begin{array}{ll}
\mathbf{H}^{(11)}[k] & \mathbf{H}^{(12)}[k] \\
\mathbf{H}^{(21)}[k] & \mathbf{H}^{(22)}[k]
\end{array}\right) \cdot\left(\begin{array}{ll}
\mathbf{x}_{\mathbf{s}}^{(1)}[k] & \mathbf{x}_{\mathbf{s}}^{(2)}[k]
\end{array}\right)+\left(\begin{array}{c}
\mathbf{n}^{(1)}[k] \\
\mathbf{n}^{(2)}[k]
\end{array}\right)
$$

so the final simplified expression results in 


$$
\mathbf{y}_{\mathrm{e}}^{(\mu)}[k]=\sum_{\nu=1}^{n_{\mathrm{T}}} \mathbf{H}^{(\mu \nu)}[k] \cdot \mathbf{x}_{\mathrm{s}}^{(\nu)}[k]+\mathbf{n}^{(\mu)}[k]
$$

where $\mu=1, \ldots, n_{\mathrm{R}}$. 


\subsection{Antennas Correlation in MIMO Systems}

As MIMO systems require a rich multipath environment for proper operation, the spatial positions of multiple receiver antennas, relative to each other and relative to their placement in the surrounding environment, may give rise to high fading correlation between antennas.

In many practical situations, however, signal correlation among the antenna elements exists in realistic environments due to poor scattering conditions. This has given an impetus for studying MIMO systems in correlated fading environments. Consequently, the effect of signal correlation on MIMO systems have been recently studied by Monte Carlo simulation in [14], [1], [30] and [34].

The antennas correlation is usually described by the antenna correlation matrix which collects the correlation coefficient between a pair of antennas [3]. Receive antennas correlation introduces undesirable effects which affect the MIMO system performance. The correlation coefficient attempts to measure the affinity of the various paths in a MIMO link. The receive antennas correlation coefficient determines the similarity between the path from a given transmit antenna to different receive antennas. The smaller the correlation coefficient the larger the possibility to benefit from the MIMO system properties [2].

This section has developed a receiver correlated MIMO system that consists of one isotropic antenna in the transmitter and four antennas in the receiver side of the radio communication system with two different antennas' distribution (linear and non-linear) in scattered environments.

\subsubsection{Antennas Correlation Characterization between two Ad- jacent Receive Antennas}

The received signal is described by the expression

$$
y_{\mathrm{e}}^{(1)}(t)=x_{\mathrm{s}}^{(1)}(t) \cdot G_{\mathrm{TX}} \cdot G_{\mathrm{RX}} \cdot A\left(d^{(1)}\right) \cdot e^{\frac{-j 2 \pi d^{(1)}}{\lambda}},
$$


where $x_{\mathrm{s}}^{(1)}(t)$ is the transmit signal, $G_{\mathrm{TX}}$ and $G_{\mathrm{RX}}$ the transmit and receive gain of the antenna which affects the channel (defined by $\left.G_{\mathrm{TX}} \cdot G_{\mathrm{RX}}=G_{1}\right), A\left(d^{(1)}\right)$ corresponds with the path attenuation for the distance $d^{(1)}$ and $\lambda$ with the wavelength.

The receive antennas correlation coefficient $\rho_{\mathrm{RX}}^{(\mu \nu)}$ determines the similitude between the path from any pair of receive antennas with respect to a given transmit antenna. The smaller the correlation coefficient the larger the possibility to benefit from the MIMO system properties. The spatial correlation coefficient between two antenna elements is a function of their spacial set-up, the linear or non-linear spatial distribution of the antenna elements and the spread angle. The antenna correlation coefficient is defined by the correlation between the received signals $y_{\mathrm{e}}^{(1)}(t)$ and $y_{\mathrm{e}}^{(2)}(t)$ and is described as follows

$$
\rho=\frac{E\left\{y_{\mathrm{e}}^{(1)}(t) \cdot y_{\mathrm{e}}^{(2)}(t)^{*}\right\}}{\left.\sqrt{E\left\{\left|y_{\mathrm{e}}^{(1)}(t)\right|^{2}\right\}} \cdot \sqrt{E\left\{\left|y_{\mathrm{e}}^{(2)}(t)\right|^{2}\right.}\right\}} .
$$

Developing the numerator the following equations are obtained

$$
\begin{gathered}
E\left\{y_{\mathrm{e}}^{(1)}(t) \cdot y_{\mathrm{e}}^{(2)}(t)^{*}\right\}= \\
=E\left\{x_{\mathrm{s}}^{(1)}(t) \cdot G_{1} \cdot A\left(d^{(1)}\right) \cdot e^{\frac{-j 2 \pi d^{(1)}}{\lambda}} \cdot x_{\mathrm{s}}^{(1)}(t)^{*} \cdot G_{2} \cdot A\left(d^{(2)}\right) \cdot e^{\frac{j 2 \pi d^{(2)}}{\lambda}}\right\} \\
=E\left\{\left|x_{\mathrm{s}}^{(1)}(t)\right|^{2} \cdot G_{1} \cdot A\left(d^{(1)}\right) \cdot G_{2} \cdot A\left(d^{(2)}\right) \cdot e^{\frac{-j 2 \pi\left[d^{(1)}-d^{(2)}\right]}{\lambda}}\right\}
\end{gathered}
$$

In order to simplify the obtained expression, some assumptions can be settled. It is supposed that the transmit signal is unitary, $\left|x_{\mathrm{s}}^{(1)}(t)\right|=1$, the antennas analysed are isotropic with unitary gain and due to $D>>d$ is also assumed that $d^{(1)} \approx d^{(2)}$ which implies that $A\left(d^{(1)}\right) \approx A\left(d^{(2)}\right) \approx A(D)$. 
After these reasonable assumptions the expression is simplified to the form

$$
E\left\{y_{\mathrm{e}}^{(1)}(t) \cdot y_{\mathrm{e}}^{(2)}(t)^{*}\right\} \approx A^{2}(D) \cdot e^{\frac{-j 2 \pi\left[d^{(1)}-d^{(2)}\right]}{\lambda}} .
$$

These assumptions are also used in the denominator obtaining the following

$$
E\left\{y_{\mathrm{e}}^{(1)}(t) \cdot y_{\mathrm{e}}^{(1)}(t)^{*}\right\} \approx A^{2}(D) \cdot e^{\frac{-j 2 \pi\left[d^{(1)}-d^{(1)}\right]}{\lambda}} \approx A^{2}(D),
$$

and

$$
E\left\{y_{\mathrm{e}}^{(2)}(t) \cdot y_{\mathrm{e}}^{(2)}(t)^{*}\right\} \approx A^{2}(D) \cdot e^{\frac{-j 2 \pi\left[d^{(2)}-d^{(2)}\right]}{\lambda}} \approx A^{2}(D)
$$

Finally, substituting in (2.62) the antenna correlation coefficient between antenna $\sharp 1$ and antenna $\sharp 2$ is obtained

$$
\rho^{(12)}=\frac{A^{2}(D) \cdot e^{\frac{-j 2 \pi\left[d^{(1)}-d^{(2)}\right]}{\lambda}}}{A^{2}(D)}=e^{\frac{-j 2 \pi\left[d^{(1)}-d^{(2)}\right]}{\lambda}}=e^{\frac{-j 2 \pi \Delta d^{(12)}}{\lambda}}=e^{-j 2 \pi \Delta d_{\lambda}^{(12)}},
$$

where $\Delta d_{\lambda}^{(12)}=\frac{\Delta d^{(12)}}{\lambda}$.

\subsubsection{Antennas Correlation in Scattered MIMO Environments}

\section{Antennas Correlation with linear Spatial Distribution}

Antennas correlation is characterized by the correlation matrix which describes the correlation coefficients between each receive-side antennas with respect to a transmit antenna [8]. The receiver correlation matrix is defined by

$$
\mathbf{R}_{\mathrm{RX}}=\left(\begin{array}{cccc}
\rho_{\mathrm{RX}}^{(11)} & \rho_{\mathrm{RX}}^{(12)} & \rho_{\mathrm{RX}}^{(13)} & \rho_{\mathrm{RX}}^{(14)} \\
\rho_{\mathrm{RX}}^{(21)} & \rho_{\mathrm{RX}}^{(22)} & \rho_{\mathrm{RX}}^{(23)} & \rho_{\mathrm{RX}}^{(24)} \\
\rho_{\mathrm{RX}}^{(31)} & \rho_{\mathrm{RX}}^{(32)} & \rho_{\mathrm{RX}}^{(33)} & \rho_{\mathrm{RX}}^{(34)} \\
\rho_{\mathrm{RX}}^{(41)} & \rho_{\mathrm{RX}}^{(42)} & \rho_{\mathrm{RX}}^{(43)} & \rho_{\mathrm{RX}}^{(44)}
\end{array}\right) .
$$

In order to characterize the receiver correlation matrix, the correlation parameter 
described in equation (2.69) is required to analyse the physical space distribution from the MIMO system.

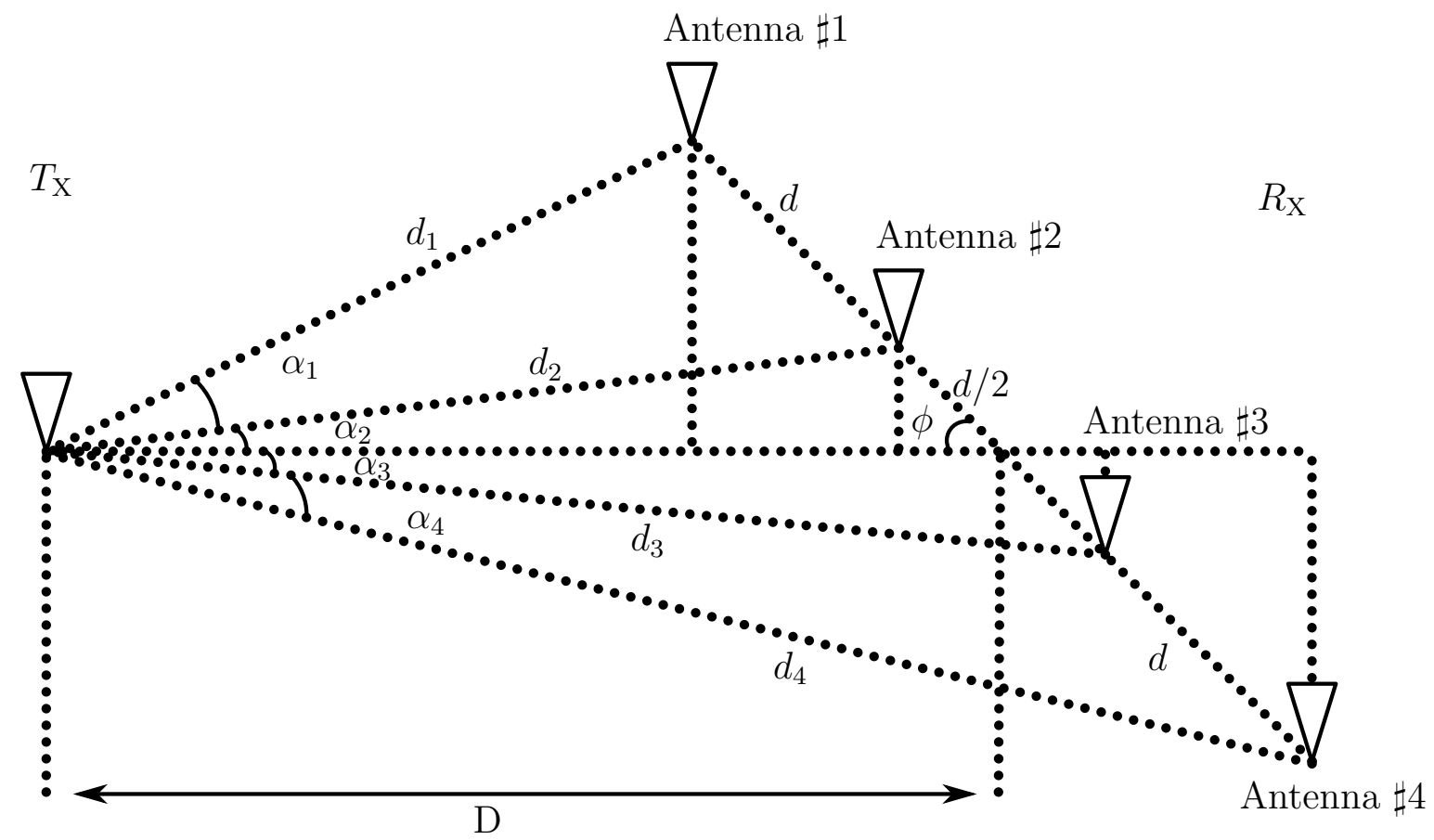

Figure 2-10: Antennas physical set-up: one transmitter-side antenna and four linearly distributed and equally spaced receiver-side antennas

From Figure 2-10 several formulas related with distance can be obtained for each pair of transmit antennas [11].

For example, the distance between antenna $\sharp 1$ and antenna $\sharp 3$ is characterized by

$$
\Delta d^{(13)}=\left(d^{(3)}-d^{(1)}\right)=\frac{2 d \cdot \cos (\phi)}{\cos \left(\frac{\varphi}{4}\right)} .
$$

Considering that the separation between transmit and receive antennas is larger compared with the transmit antennas separation $(D>>d)$, it is assumed that $\alpha_{1} \approx$ $\alpha_{2} \approx \alpha_{3} \approx \alpha_{4} \approx \frac{\varphi}{4}$ where $\varphi$ is called the spread angle. In order to simplify the equations, it is possible to assume that $\cos \left(\frac{\varphi}{4}\right) \approx 1$.

After this spread angle assumption, the distance between antenna $\sharp 1$ and antenna $\sharp 3$ is denoted by 


$$
\Delta d^{(13)}=d^{(3)}-d^{(1)}=2 d \cdot \cos (\phi)
$$

For an arbitrary scatter, the difference of the distances which affects the correlation coefficients is given by $\Delta d=2 d \cdot \cos (\phi+\xi)$, where $\xi$ is a random variable that must be appropriately modelled. In order to select an appropriate model for $\xi$, it is considered that most of the scatters are concentrated around the mean scatter and the probability of receiving scatters for a certain angle $\xi$ follows a normal distribution with mean $\hat{\mu}=0$ and standard deviation $\sigma_{\xi}=1[11]$.

In consequence, the correlation parameter $\rho_{\mathrm{RX}}^{(\mu \nu)}$ becomes

$$
\begin{gathered}
E\left\{x_{\mathrm{s}}^{(1)}(t) * x_{\mathrm{s}}^{(3)}(t)\right\}= \\
=E\left\{\left|x_{\mathrm{s}}^{(1)}(t)\right|^{2} \cdot e^{-j 2 \pi \frac{d^{(3)}-d^{(1)}}{\lambda}}\right\} \cdot A\left(d^{(1)}\right) \cdot A\left(d^{(3)}\right) \\
=E\left\{e^{-j 2 \pi 2 d \frac{\cos (\phi+\xi)}{\lambda}}\right\} \cdot A\left(d^{(1)}\right) \cdot A\left(d^{(3)}\right) \\
=E\left\{e^{-j 2 \pi 2 d_{\lambda} \cos (\phi+\xi)}\right\} \cdot A\left(d^{(1)}\right) \cdot A\left(d^{(3)}\right),
\end{gathered}
$$

where $d_{\lambda}=\frac{d}{\lambda}$.

$$
E\left\{y_{\mathrm{e}}^{(1)}(t) \cdot y_{\mathrm{e}}^{(1)}(t)^{*}\right\}=A^{2}(D) \cdot e^{\frac{-j 2 \pi\left[d^{(1)}-d^{(1)}\right]}{\lambda}} \approx A^{2}(D)
$$

and

$$
E\left\{y_{\mathrm{e}}^{(3)}(t) \cdot y_{\mathrm{e}}^{(3)}(t)^{*}\right\}=A^{2}(D) \cdot e^{\frac{-j 2 \pi\left[d^{(3)}-d^{(3)}\right]}{\lambda}} \approx A^{2}(D)
$$

Thus, substituting in (2.69), the correlation coefficient is obtained

$$
\rho_{\mathrm{RX}}^{(13)}\left(\phi, \xi, d_{\lambda}\right)=E\left\{e^{j 2 \pi 2 d_{\lambda} \cos (\phi+\xi)}\right\}
$$


Considering the parameter $\xi$ as a normal distributed random variable, the computation of the expectation results in

$$
\rho_{\mathrm{RX}}^{(13)}\left(\phi, \xi, d_{\lambda}\right)=\int_{-\infty}^{\infty} e^{j 2 \pi 2 d_{\lambda} \cos (\phi+\xi)} \cdot p(\xi) \cdot d(\xi)
$$

where $p(\xi)$ is the probability distribution function of the scatters' angle $\xi$

$$
p(\xi)=\frac{1}{\sqrt{2 \pi \sigma_{\xi}^{2}}} \cdot e^{\frac{-\xi^{2}}{2 \sigma_{\xi}^{2}}} .
$$

Developing the expression $\cos (\phi+\xi)$, it is assumed that $\xi$ is small enough, so $\cos (\xi) \approx 1$ and $\sin (\xi) \approx \xi$

$$
\cos (\phi+\xi)=\cos (\phi) \cos (\xi)-\sin (\phi) \sin (\xi) \simeq \cos (\phi)-\xi \sin (\xi)
$$

Taking into account the previous equations, the following integral has to be solved

$$
\int_{-\infty}^{\infty} e^{j 2 \pi 2 d_{\lambda}[\cos (\phi)-\xi \sin (\xi)]} \cdot \frac{1}{\sqrt{2 \pi \sigma_{\xi}^{2}}} \cdot e^{\frac{-\xi^{2}}{2 \sigma_{\xi}^{2}}} \cdot d(\xi)
$$

considering the expression

$$
\int_{-\infty}^{\infty} e^{\frac{-a x^{2}}{2}+j b x} d x=\left(\frac{2 \pi}{a}\right)^{\frac{1}{2}} \cdot e^{\frac{-b^{2}}{2 a}}
$$

where it is identified $a=\frac{1}{\sigma_{\xi}^{2}}$ and $b=2 \pi 2 d_{\lambda} \sin (\phi)$.

Finally, the resulting integral becomes the correlation coefficient

$$
\rho_{\mathrm{RX}}^{(13)}=e^{-j 2 \pi 2 d_{\lambda} \cos (\phi)} \cdot e^{\frac{-1}{2}\left[2 \pi 2 d_{\lambda} \sin (\phi) \sigma_{\xi}\right]^{2}} .
$$

Further distances between each pair of antennas are denoted by

$$
\Delta d^{(11)}=\Delta d^{(22)}=\Delta d^{(33)}=\Delta d^{(44)}=0 .
$$




$$
\begin{aligned}
& \Delta d^{(12)}=\Delta d^{(21)}=d \cdot \cos (\phi) \\
& \Delta d^{(23)}=\Delta d^{(32)}=d \cdot \cos (\phi) \\
& \Delta d^{(43)}=\Delta d^{(34)}=d \cdot \cos (\phi)
\end{aligned}
$$

$$
\begin{gathered}
\Delta d^{(13)}=\Delta d^{(31)}=2 d \cdot \cos (\phi) \\
\Delta d^{(24)}=\Delta d^{(42)}=2 d \cdot \cos (\phi)
\end{gathered}
$$

$$
\Delta d^{(14)}=\Delta d^{(41)}=3 d \cdot \cos (\phi)
$$

Applying the same development and taking into consideration the distances obtained, the previously calculated integral and the correlation matrix $\mathbf{R}_{\mathrm{Rx}}$ described at the beginning of this section, it is possible to assert that the antennas correlation coefficients are given by the next general expression [8]

$$
\rho_{\mathrm{RX}}^{(\mu \nu)}\left(\phi, \sigma_{\xi}\right)=e^{-j 2 \pi|\mu-\nu| d_{\lambda} \cos (\phi)} \cdot e^{\frac{-1}{2}\left[2 \pi|\mu-\nu| d_{\lambda} \sin (\phi) \sigma_{\xi}\right]^{2}}
$$

where $\mu=1, \ldots, n_{\mathrm{R}}$ and $\nu=1, \ldots, n_{\mathrm{T}}$.

\section{Antennas Correlation with non-linear Spatial Distribution}

In this section the results obtained for $(4 \times 4)$ MIMO correlated linear distributed system are extended to a non-linear distributed system [12].

The correlation matrix is defined by 


$$
\mathbf{R}_{\mathrm{RX}}=\left(\begin{array}{cccc}
\rho_{\mathrm{RX}}^{(11)} & \rho_{\mathrm{RX}}^{(12)} & \rho_{\mathrm{RX}}^{(13)} & \rho_{\mathrm{RX}}^{(14)} \\
\rho_{\mathrm{RX}}^{(21)} & \rho_{\mathrm{RX}}^{(22)} & \rho_{\mathrm{RX}}^{(23)} & \rho_{\mathrm{RX}}^{(24)} \\
\rho_{\mathrm{RX}}^{(31)} & \rho_{\mathrm{RX}}^{(32)} & \rho_{\mathrm{RX}}^{(33)} & \rho_{\mathrm{RX}}^{(34)} \\
\rho_{\mathrm{RX}}^{(41)} & \rho_{\mathrm{RX}}^{(42)} & \rho_{\mathrm{RX}}^{(43)} & \rho_{\mathrm{RX}}^{(44)}
\end{array}\right)
$$

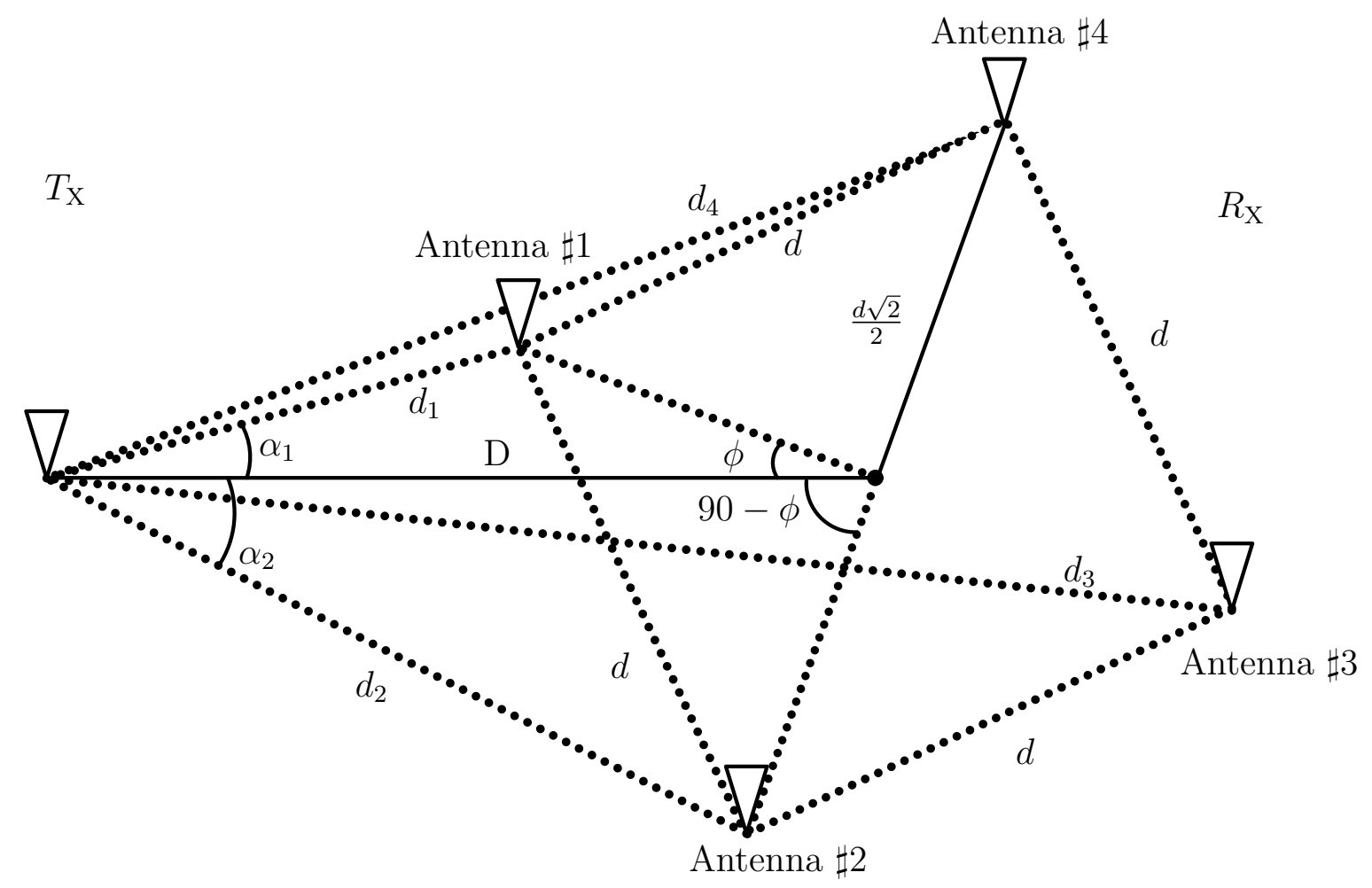

Figure 2-11: Antennas physical set-up: one transmitter-side antenna and four nonlinearly distributed and equally spaced receiver-side antennas

Figure 2-11 shows the physical distribution and several formulas related with the distance between antennas and spread angles that can be obtained for each pair of transmit antennas [11].

Considering the distance between the antenna $\sharp 1$ and antenna $\sharp 2$ in the form

$$
\begin{gathered}
d^{(1)} \cdot \cos \left(\alpha_{1}\right)=D-\frac{\sqrt{2}}{2} \cdot d \cdot \cos (\phi), \\
d^{(2)} \cdot \cos \left(\alpha_{2}\right)=D-\frac{\sqrt{2}}{2} \cdot d \cdot \cos \left(\frac{\pi}{2}-\phi\right),
\end{gathered}
$$


the difference between them is calculated

$$
\begin{aligned}
\left(d^{(1)}-d^{(2)}\right) \cdot \cos \left(\alpha_{12}\right) & =-\frac{\sqrt{2}}{2} \cdot d \cdot \cos (\phi)+\frac{\sqrt{2}}{2} \cdot d \cdot \cos \left(\frac{\pi}{2}-\phi\right) \\
\left(d^{(1)}-d^{(2)}\right) \cdot \cos \left(\alpha_{12}\right) & =-\frac{\sqrt{2}}{2} \cdot d \cdot[\cos (\phi)+\sin (\phi)] \\
d^{(1)}-d^{(2)} & =-\frac{\sqrt{2}}{2} \cdot d \cdot \frac{[\cos (\phi)+\sin (\phi)]}{\cos \left(\alpha_{12}\right)} .
\end{aligned}
$$

Furthermore, if $D>>d$ it is possible to assume that $\alpha_{1} \approx \alpha_{2}$, and consequently, $\cos \left(\alpha_{12}\right) \approx 1$, applying the same assumption to the others' pairs of antennas.

$$
\Delta d^{(12)}=\frac{-\sqrt{2}}{2} \cdot d \cdot[\cos (\phi)+\sin (\phi)]
$$

For introducing an arbitrary scatter, the distance difference which affects the correlation coefficients is given by $\Delta d^{(12)}=\frac{-\sqrt{2}}{2} \cdot d \cdot[\cos (\phi+\xi)+\sin (\phi+\xi)]$.

In consequence, the correlation parameter $\rho_{\mathrm{RX}}^{(\mu \nu)}$ results in

$$
\begin{gathered}
E\left\{x_{\mathrm{s}}^{(1)}(t) * x_{\mathrm{s}}^{(2)}(t)\right\}=E\left\{\left|x_{\mathrm{s}}^{(1)}(t)\right|^{2} \cdot e^{-j 2 \pi \frac{\Delta d^{(12)}}{\lambda}}\right\} \cdot A\left(d^{(1)}\right) \cdot A\left(d^{(2)}\right) \\
=E\left\{e^{+j 2 \pi \frac{\sqrt{2}}{2} d \frac{\cos (\phi+\xi)+\sin (\phi+\xi)}{\lambda}}\right\} \cdot A\left(d^{(1)}\right) \cdot A\left(d^{(2)}\right) \\
=E\left\{e^{+j 2 \pi \frac{\sqrt{2}}{2} d_{\lambda} \cdot[\cos (\phi+\xi)+\sin (\phi+\xi)]}\right\} \cdot A\left(d^{(1)}\right) \cdot A\left(d^{(2)}\right)
\end{gathered}
$$

where $d_{\lambda}=\frac{d}{\lambda}$.

$$
E\left\{y_{\mathrm{e}}^{(1)}(t) \cdot y_{\mathrm{e}}^{(1)}(t)^{*}\right\}=A^{2}(D) \cdot e^{\frac{-j 2 \pi\left[\Delta d^{(11)}\right]}{\lambda}} \approx A^{2}(D)
$$

and 


$$
E\left\{y_{\mathrm{e}}^{(2)}(t) \cdot y_{\mathrm{e}}^{(2)}(t)^{*}\right\}=A^{2}(D) \cdot e^{\frac{-j 2 \pi\left[\Delta d^{(22)}\right]}{\lambda}} \approx A^{2}(D)
$$

Hence, substituting in (2.69) it is obtained the correlation coefficient

$$
\rho_{\mathrm{RX}}^{(12)}\left(\phi, \xi, d_{\lambda}\right)=E\left\{e^{j 2 \pi \frac{\sqrt{2}}{2}} d_{\lambda} \cdot[\cos (\phi+\xi)+\sin (\phi+\xi)]\right\} .
$$

Considering the parameter $\xi$ as a normal distributed random variable, the computation of the expectation becomes

$$
\rho_{\mathrm{RX}}^{(12)}\left(\phi, \xi, d_{\lambda}\right)=\int_{-\infty}^{\infty} e^{j 2 \pi \frac{\sqrt{2}}{2} d_{\lambda} \cdot[\cos (\phi+\xi)+\sin (\phi+\xi)]} \cdot p(\xi) \cdot d(\xi),
$$

where $p(\xi)$ is the probability distribution function of the scatters' angle $\xi$

$$
p(\xi)=\frac{1}{\sqrt{2 \pi \sigma_{\xi}^{2}}} \cdot e^{\frac{-\xi^{2}}{2 \sigma_{\xi}^{2}}} .
$$

Developing the expressions $\cos (\phi+\xi)$ and $\sin (\phi+\xi)$ and assuming that $\xi$ is small enough, $\cos (\xi) \approx 1$ and $\sin (\xi) \approx \xi$,

$$
\begin{aligned}
& \cos (\phi+\xi)=\cos (\phi) \cos (\xi)-\sin (\phi) \sin (\xi) \simeq \cos (\phi)-\xi \sin (\xi), \\
& \sin (\phi+\xi)=\sin (\phi) \cos (\xi)+\cos (\phi) \sin (\xi) \simeq \sin (\phi)+\xi \cos (\xi),
\end{aligned}
$$

combining both expressions

$$
\cos (\phi+\xi)+\sin (\phi+\xi)=\xi \cdot[\cos (\phi)-\sin (\phi)]+\cos (\phi)+\sin (\phi)
$$

Taking into account the previous equations, the following integral is required to be solved 


$$
\int_{-\infty}^{\infty} e^{j 2 \pi \frac{\sqrt{2}}{2} d_{\lambda}[\xi \cdot[\cos (\phi)-\sin (\phi)]+\cos (\phi)+\sin (\phi)]} \cdot \frac{1}{\sqrt{2 \pi \sigma_{\xi}^{2}}} \cdot e^{\frac{-\xi^{2}}{2 \sigma_{\xi}^{2}}} \cdot d(\xi)
$$

considering the expression

$$
\int_{-\infty}^{\infty} e^{\frac{-a x^{2}}{2}+j b x} d x=\left(\frac{2 \pi}{a}\right)^{\frac{1}{2}} \cdot e^{\frac{-b^{2}}{2 a}}
$$

where it is identified $a=\frac{1}{\sigma \xi^{2}}$ and $b=-2 \pi \frac{\sqrt{2}}{2} d_{\lambda} \cdot[\cos (\phi)-\sin (\phi)]$.

Finally, the resulting integral becomes

$$
\rho_{\mathrm{RX}}^{(12)}=e^{+j 2 \pi d_{\lambda} \frac{\sqrt{2}}{2}[\sin (\phi)+\cos (\phi)]} \cdot e^{\frac{-1}{2} \cdot\left[2 \pi d_{\lambda} \frac{\sqrt{2}}{2}[\cos (\phi)-\sin (\phi)] \sigma_{\xi}\right]^{2}} .
$$

The same mathematical process for the others' pairs of antennas is developed with the aim to achieve the antennas distances relationships.

$$
\begin{gathered}
\Delta d^{(34)}=\frac{\sqrt{2}}{2} \cdot d \cdot[\cos (\phi)+\sin (\phi)], \\
\Delta d^{(13)}=-\sqrt{2} \cdot d \cdot \cos (\phi), \\
\Delta d^{(24)}=\sqrt{2} \cdot d \cdot \sin (\phi), \\
\Delta d^{(14)}=\frac{\sqrt{2}}{2} \cdot d \cdot[\sin (\phi)-\cos (\phi)], \\
\Delta d^{(23)}=\frac{\sqrt{2}}{2} \cdot d \cdot[\sin (\phi)-\cos (\phi)],
\end{gathered}
$$

and the relationship between cross-correlated parameters is the conjugate operator

$$
\Delta d^{(m n)}=\left(\Delta d^{(n m)}\right)^{*} .
$$


If the same development is applied considering the distances obtained, the integral calculated previously and the correlation matrix $\mathbf{R}_{\mathrm{Rx}}$ described at the beginning of this section, it is possible to assert that the antennas correlation coefficients are given by the next expressions

$$
\begin{aligned}
& \rho_{\mathrm{RX}}^{(13)}=e^{+j 2 \pi d_{\lambda} \sqrt{2} \cos (\phi)} \cdot e^{\left.\frac{-1}{2} \cdot\left[2 \pi d_{\lambda} \sqrt{2} \sin (\phi)\right] \sigma_{\xi}\right]^{2}} . \\
& \rho_{\mathrm{RX}}^{(14)}=e^{-j 2 \pi d_{\lambda} \frac{\sqrt{2}}{2}[\sin (\phi)-\cos (\phi)]} \cdot e^{\frac{-1}{2} \cdot\left[2 \pi d_{\lambda} \frac{\sqrt{2}}{2}[\cos (\phi)+\sin (\phi)] \sigma_{\xi}\right]^{2}} . \\
& \rho_{\mathrm{RX}}^{(21)}=e^{-j 2 \pi d_{\lambda} \frac{\sqrt{2}}{2}[\sin (\phi)+\cos (\phi)]} \cdot e^{\frac{-1}{2} \cdot\left[2 \pi d_{\lambda} \frac{\sqrt{2}}{2}[\cos (\phi)-\sin (\phi)] \sigma_{\xi}\right]^{2}} . \\
& \rho_{\mathrm{RX}}^{(23)}=e^{-j 2 \pi d_{\lambda} \frac{\sqrt{2}}{2}[\sin (\phi)-\cos (\phi)]} \cdot e^{\frac{-1}{2} \cdot\left[2 \pi d_{\lambda} \frac{\sqrt{2}}{2}[\cos (\phi)+\sin (\phi)] \sigma_{\xi}\right]^{2}} . \\
& \rho_{\mathrm{RX}}^{(24)}=e^{-j 2 \pi d_{\lambda} \sqrt{2} \sin (\phi)} \cdot e^{\frac{-1}{2} \cdot\left[2 \pi d_{\lambda} \sqrt{2} \cos (\phi) \sigma_{\xi}\right]^{2}} . \\
& \rho_{\mathrm{RX}}^{(31)}=e^{-j 2 \pi d_{\lambda} \sqrt{2} \cos (\phi)} \cdot e^{\left.\frac{-1}{2} \cdot\left[2 \pi d_{\lambda} \sqrt{2} \sin (\phi)\right] \sigma_{\xi}\right]^{2}} . \\
& \rho_{\mathrm{RX}}^{(32)}=e^{-j 2 \pi d_{\lambda} \frac{\sqrt{2}}{2}[\cos (\phi)-\sin (\phi)]} \cdot e^{\frac{-1}{2} \cdot\left[2 \pi d_{\lambda} \frac{\sqrt{2}}{2}[\cos (\phi)+\sin (\phi)] \sigma_{\xi}\right]^{2}} . \\
& \rho_{\mathrm{RX}}^{(34)}=e^{-j 2 \pi d_{\lambda} \frac{\sqrt{2}}{2}[\sin (\phi)+\cos (\phi)]} \cdot e^{\frac{-1}{2} \cdot\left[2 \pi d_{\lambda} \frac{\sqrt{2}}{2}[\cos (\phi)-\sin (\phi)] \sigma_{\xi}\right]^{2}} . \\
& \rho_{\mathrm{RX}}^{(41)}=e^{-j 2 \pi d_{\lambda} \frac{\sqrt{2}}{2}[\cos (\phi)-\sin (\phi)]} \cdot e^{\frac{-1}{2} \cdot\left[2 \pi d_{\lambda} \frac{\sqrt{2}}{2}[\cos (\phi)+\sin (\phi)] \sigma_{\xi}\right]^{2}} . \\
& \rho_{\mathrm{RX}}^{(42)}=e^{+j 2 \pi d_{\lambda} \sqrt{2} \sin (\phi)} \cdot e^{\frac{-1}{2} \cdot\left[2 \pi d_{\lambda} \sqrt{2} \cos (\phi) \sigma_{\xi}\right]^{2}} .
\end{aligned}
$$




$$
\rho_{\mathrm{RX}}^{(43)}=e^{+j 2 \pi d_{\lambda} \frac{\sqrt{2}}{2}[\sin (\phi)+\cos (\phi)]} \cdot e^{\frac{-1}{2} \cdot\left[2 \pi d_{\lambda} \frac{\sqrt{2}}{2}[\cos (\phi)-\sin (\phi)] \sigma_{\xi}\right]^{2}} .
$$

\subsubsection{Antennas correlation on a $(4 \times 4)$ MIMO link}

The approach to include the antenna signal correlation into the MIMO channel model with $n_{\mathrm{T}}$ transmit and $n_{\mathrm{R}}$ receive antennas for Rayleigh flat-fading like channels is given by [8] and results in

$$
\operatorname{vec}(\mathbf{H})=\mathbf{R}_{\mathrm{HH}}^{\left(\frac{1}{2}\right)} \cdot \operatorname{vec}(\mathbf{G})
$$

where $\mathbf{G}$ is an $\left(n_{\mathrm{R}} \times n_{\mathrm{T}}\right)$ uncorrelated channel matrix with independent and identically distributed (i.i.d) complex Rayleigh elements and $v e c(\cdot)$ the operator which stacks the matrix $\mathbf{G}$ into a vector column-wise. The matrix $\mathbf{R}_{\mathrm{HH}}$ that describes the overall correlation is defined as

$$
\mathbf{R}_{\mathrm{HH}}=E\left\{\operatorname{vec}(\mathbf{H}) \cdot \operatorname{vec}(\mathbf{H})^{* \mathrm{~T}}\right\}
$$

Assuming that the correlation introduced by the antenna elements at the transmitter side is independent from the correlation introduced by the antenna elements at the receiver side, the correlation matrix can be defined over the transmitter side correlation matrix $\mathbf{R}_{\mathrm{TX}}$ as well as the receiver side correlation matrix $\mathbf{R}_{\mathrm{RX}}$. In this case the matrix $\mathbf{R}_{\mathrm{HH}}$ results in

$$
\mathbf{R}_{\mathrm{HH}}=\mathbf{R}_{\mathrm{TX}} \otimes \mathbf{R}_{\mathrm{RX}}
$$

where $\otimes$ represents the Kronecker product.

In this chapter, it is assumed that there is no correlation between the antennas at the transmitter side, so $\mathbf{R}_{\mathrm{TX}}$ results in 


$$
\mathbf{R}_{\mathrm{TX}}=\left(\begin{array}{cccc}
\rho_{\mathrm{TX}}^{(11)} & \rho_{\mathrm{TX}}^{(12)} & \rho_{\mathrm{TX}}^{(13)} & \rho_{\mathrm{TX}}^{(14)} \\
\rho_{\mathrm{TX}}^{(21)} & \rho_{\mathrm{TX}}^{(22)} & \rho_{\mathrm{TX}}^{(23)} & \rho_{\mathrm{TX}}^{(24)} \\
\rho_{\mathrm{TX}}^{(31)} & \rho_{\mathrm{TX}}^{(32)} & \rho_{\mathrm{TX}}^{(33)} & \rho_{\mathrm{TX}}^{(34)} \\
\rho_{\mathrm{TX}}^{(41)} & \rho_{\mathrm{TX}}^{(42)} & \rho_{\mathrm{TX}}^{(43)} & \rho_{\mathrm{TX}}^{(44)}
\end{array}\right)=\left(\begin{array}{cccc}
1 & 0 & 0 & 0 \\
0 & 1 & 0 & 0 \\
0 & 0 & 1 & 0 \\
0 & 0 & 0 & 1
\end{array}\right)
$$

and the receiver side correlation matrix $\mathbf{R}_{\mathrm{RX}}$ is given by

$$
\mathbf{R}_{\mathrm{RX}}=\left(\begin{array}{cccc}
\rho_{\mathrm{RX}}^{(11)} & \rho_{\mathrm{RX}}^{(12)} & \rho_{\mathrm{RX}}^{(13)} & \rho_{\mathrm{RX}}^{(14)} \\
\rho_{\mathrm{RX}}^{(21)} & \rho_{\mathrm{RX}}^{(22)} & \rho_{\mathrm{RX}}^{(23)} & \rho_{\mathrm{RX}}^{(24)} \\
\rho_{\mathrm{RX}}^{(31)} & \rho_{\mathrm{RX}}^{(32)} & \rho_{\mathrm{RX}}^{(33)} & \rho_{\mathrm{RX}}^{(34)} \\
\rho_{\mathrm{RX}}^{(41)} & \rho_{\mathrm{RX}}^{(42)} & \rho_{\mathrm{RX}}^{(43)} & \rho_{\mathrm{RX}}^{(44)}
\end{array}\right)=\left(\begin{array}{cccc}
1 & \rho_{\mathrm{RX}}^{(12)} & \rho_{\mathrm{RX}}^{(13)} & \rho_{\mathrm{RX}}^{(14)} \\
\rho_{\mathrm{RX}}^{(21)} & 1 & \rho_{\mathrm{RX}}^{(23)} & \rho_{\mathrm{RX}}^{(24)} \\
\rho_{\mathrm{RX}}^{(31)} & \rho_{\mathrm{RX}}^{(32)} & 1 & \rho_{\mathrm{RX}}^{(34)} \\
\rho_{\mathrm{RX}}^{(41)} & \rho_{\mathrm{RX}}^{(42)} & \rho_{\mathrm{RX}}^{(43)} & 1
\end{array}\right)
$$

Simulations In Figure 2-12, Figure 2-14, Figure 2-15, Figure 2-16 and Figure 217 the CCDFs of the layer-specific distribution for uncorrelated, weak and strong correlated frequency non-selective $(4 \times 4)$ MIMO channel with linear and non-linear spatial distribution are plotted. The CCDF curves represent the probability of having singular values higher than a specific $\gamma$ value.

The established parameters in the simulation performance are listed on Table 2.1 and Table 2.2 in order to simulate a weak correlation (CM-1) and a strong correlation (CM-2). A non-correlated one is set for comparison reasons (CM-3).

Table 2.1: Channel Profiles for Linear Distribution

\begin{tabular}{cccc}
\hline Description & Arrival Angle & Standard Deviation & Distance \\
\hline CM-1 & $\phi=30^{\circ} \mathrm{rad}$ & $\sigma_{\xi}=1$ & $d_{\lambda}=1$ \\
CM-2 & $\phi=30^{\circ} \mathrm{rad}$ & $\sigma_{\xi}=1$ & $d_{\lambda}=0.25$ \\
CM-3 & - & - & - \\
\hline
\end{tabular}

The study of the CCDF curves reports additional information related to the effect produces by the correlation between antennas over the MIMO communication links.

The following figures shows how the probability of the singular values is modified 
Table 2.2: Channel Profiles for Non-linear Distribution

\begin{tabular}{cccc}
\hline Description & Arrival Angle & Standard Deviation & Distance \\
\hline CM-1 & $\phi=90^{\circ} \mathrm{rad}$ & $\sigma_{\xi}=1$ & $d_{\lambda}=1$ \\
$\mathrm{CM}-2$ & $\phi=90^{\circ} \mathrm{rad}$ & $\sigma_{\xi}=1$ & $d_{\lambda}=0.25$ \\
$\mathrm{CM}-3$ & - & - & - \\
\hline
\end{tabular}

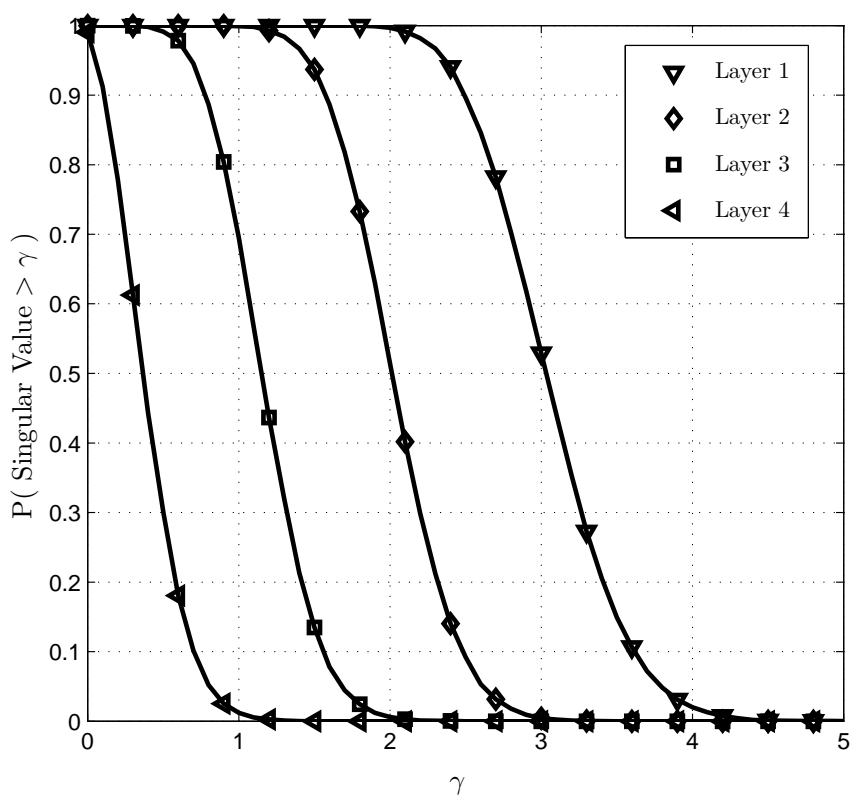

Figure 2-12: CCDF of the layer-specific distribution for uncorrelated frequency nonselective $(4 \times 4)$ MIMO channel with linear spatial distribution 
by the correlation effect becoming more relevant to ones' layers than others. Figure 214 compares the effect of the correlation in a linear spacial distribution due to nocorrelated CCDF curves (solid lines) that are uniformly distributed and the correlated CCDF curves (dashed and dotted lines) not at all. The correlated CCDF curves in a weak (CM-1) or in a strong (CM-2) correlated scenario show how the probability of having a predominant layer is increased compared with a non-correlated scenario (CM-3). For example, if are compared the singular vales for layer $\sharp 4$ in (CM-1) and (CM-3) for a certain probability, $10 \%$ are fixed, the singular value for layer $\sharp 4$ in (CM-3) takes 3.6 and in (CM-1) takes 4.8 which is clearly a higher probability. This result is extended to (CM-2).

A similar conclusion can be obtained by analysing the CCDF curves from Figure 215 which shows that the impact of the correlation in a non-linear spacial distribution is even higher than in a linear spacial distribution.

From Figure 2-16 and Figure 2-17 the ratio between layer $\sharp 1$ and $\sharp 4$ is analysed. This higher ratio compared with (CM-3) ratio means that given a fixed available power much more power at the transmitter-side should be provided to layer $\sharp 4$ to get the expected performance in a correlated scenario. 


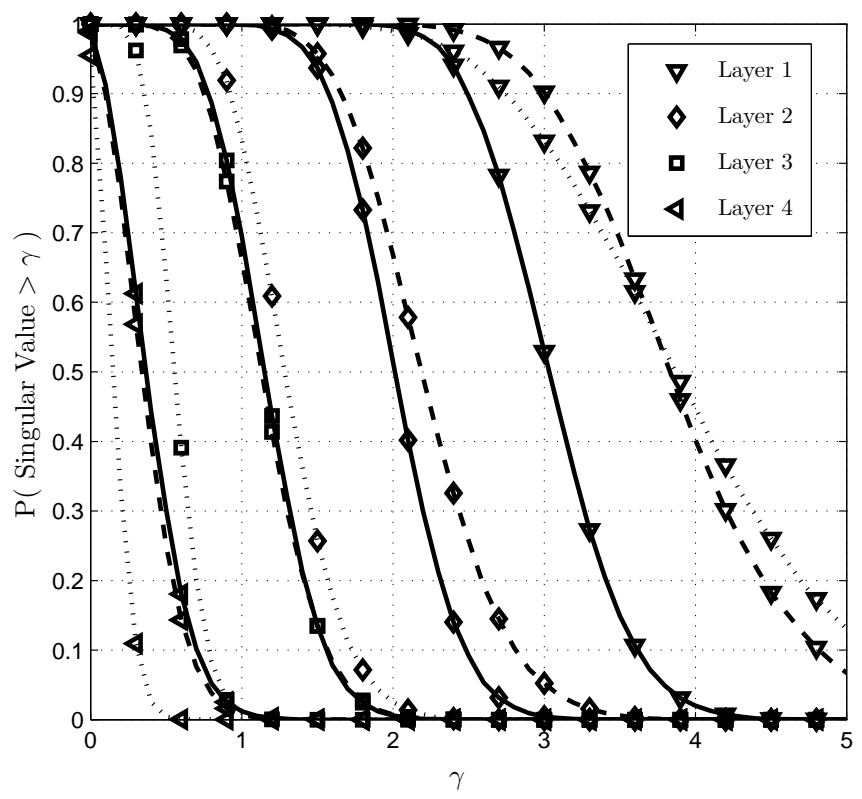

Figure 2-13: CCDF of the layer-specific distribution for uncorrelated (solid line), the weak correlated (dashed line) and the strong correlated (dotted line) frequency non-selective $(4 \times 4)$ MIMO channel with linear spatial distribution. The established parameters in the simulation are $\phi=30^{\circ} \mathrm{rad}, \sigma_{\xi}=1$ and $d_{\lambda}=1$ in order to simulate a weak correlation (CM-1), and $d_{\lambda}=0.25$ for strong correlation (CM-2). A noncorrelated one is set for comparison reasons (CM-3) 


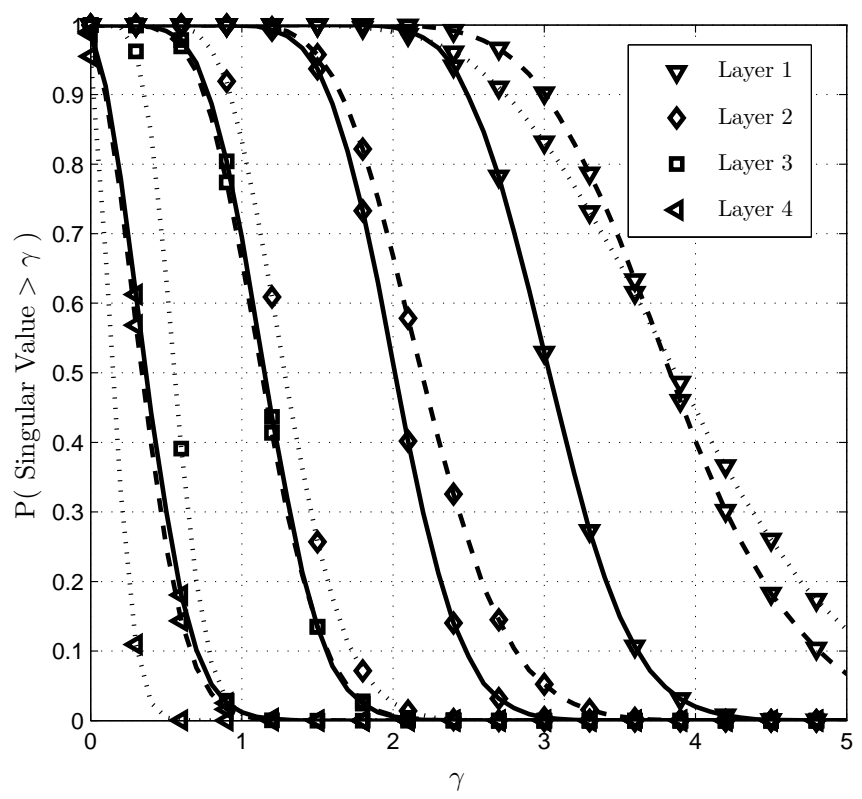

Figure 2-14: CCDF of the layer-specific distribution for uncorrelated (solid line), the weak correlated (dashed line) and the strong correlated (dotted line) frequency non-selective $(4 \times 4)$ MIMO channel with linear spatial distribution. The established parameters in the simulation are $\phi=30^{\circ} \mathrm{rad}, \sigma_{\xi}=1$ and $d_{\lambda}=1$ in order to simulate a weak correlation (CM-1), and $d_{\lambda}=0.25$ for strong correlation (CM-2). A no correlated one is set for comparison reasons (CM-3)

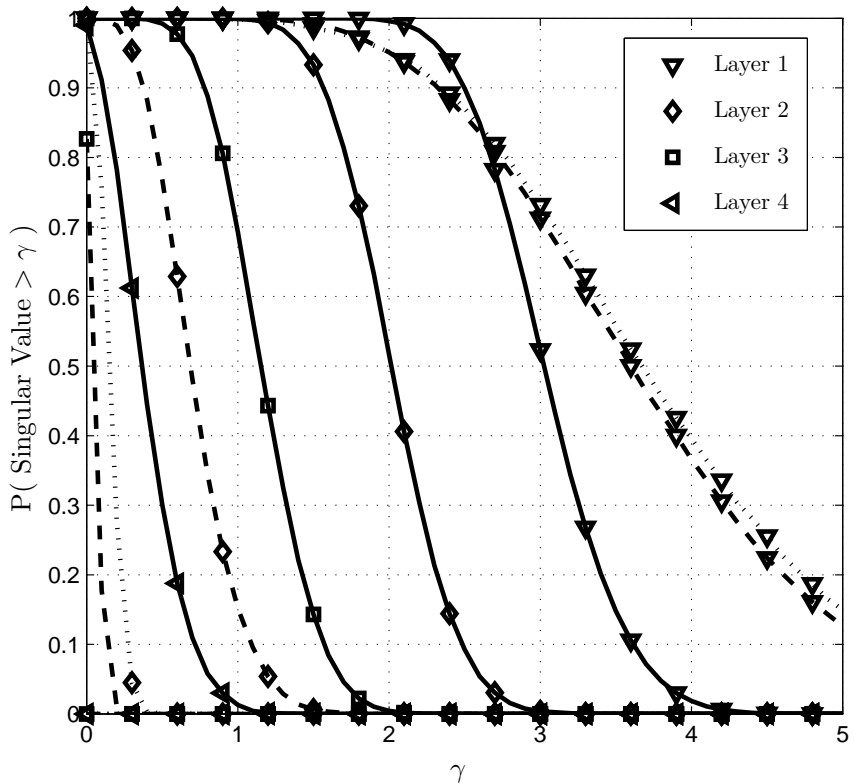

Figure 2-15: CCDF of the layer-specific distribution for uncorrelated (solid line), the weak correlated (dashed line) and the strong correlated (dotted line) frequency non-selective $(4 \times 4)$ MIMO channel with non-linear distribution. The established parameters in the simulation were $\phi=90^{\circ} \mathrm{rad}, \sigma_{\xi}=1$ and $d_{\lambda}=1$ in order to simulate a weak correlation (CM-1) and $d_{\lambda}=0.25$ for strong correlation (CM-2) 


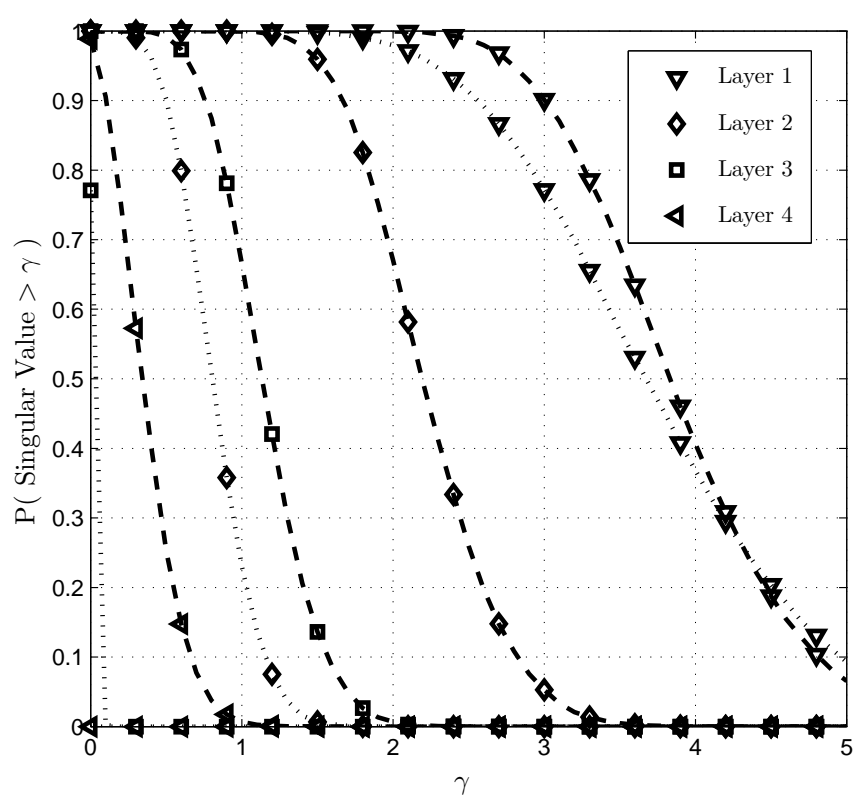

Figure 2-16: CCDF of the layer-specific distribution for the weak correlated (dashed line) with linear distribution and the weak correlated (dotted line) with non-linear distribution for a frequency non-selective $(4 \times 4)$ MIMO channel. The established parameters in the simulation were $\phi=30^{\circ} \mathrm{rad}, \sigma_{\xi}=1$ and $d_{\lambda}=1$ in order to simulate a weak correlation and $d_{\lambda}=0.25$ for strong correlation

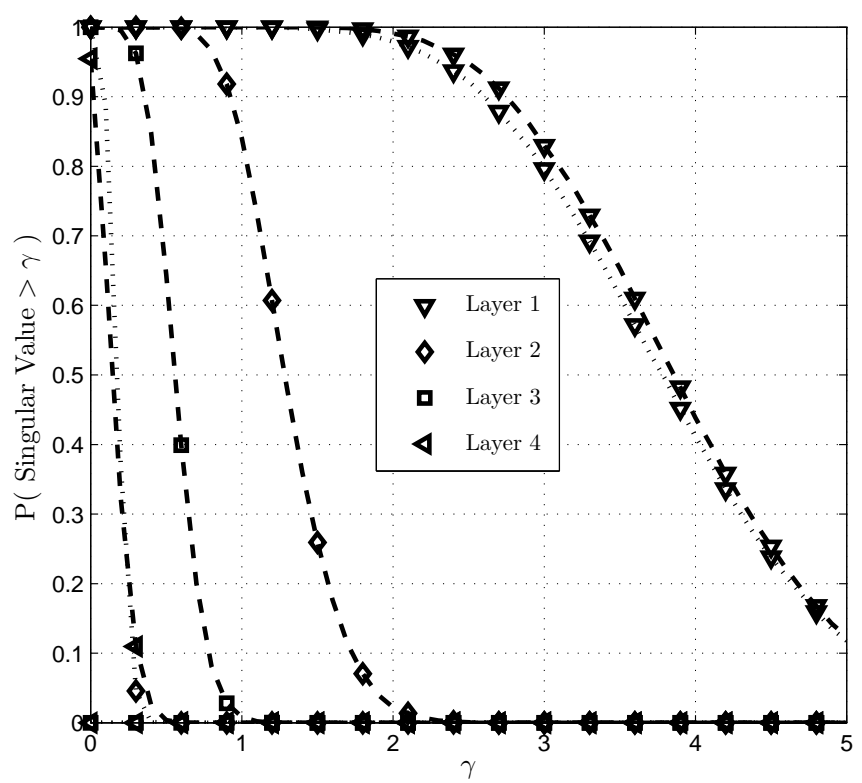

Figure 2-17: CCDF of the layer-specific distribution for the strong correlated (dashed line) with linear distribution and the strong correlated (dotted line with non-linear distribution for a frequency non-selective $(4 \times 4)$ MIMO channel. The established parameters in the simulation were $\phi=30^{\circ} \mathrm{rad}, \sigma_{\xi}=1$ and $d_{\lambda}=1$ in order to simulate a weak correlation and $d_{\lambda}=0.25$ for strong correlation 


\subsection{Chapter Conclusions}

In this chapter at the doctoral thesis the impact of the correlation between receiver antennas over the characterization of the MIMO channel has been analysed.

In order to accomplish this analysis, the CCDF curves of each active layer in every $(4 \times 4)$ MIMO scenario described have been developed.

Due to these simulations the strong effect of the correlation over the quality criteria and capacity, highlighted in Figure 2-18, in MIMO systems is shown, and the huge dependence correlation presents with the antenna distance and the topology of the antennas. The higher the correlation effects, the higher is the predominance of strong layers with large valued singular values.

As a conclusion, due to the effects of distance between antennas and its spatial distribution, it is not required to activate all layers in a MIMO communication system in order to obtain the best performance.

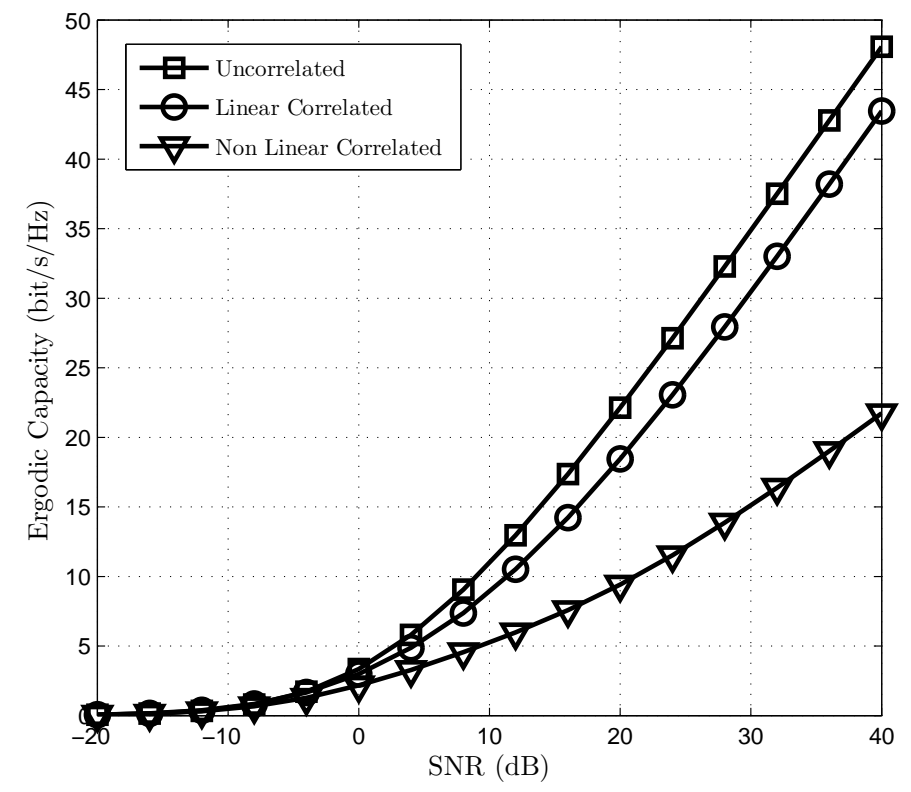

Figure 2-18: Capacity comparison between uncorrelated and correlated (linear and non-linear distribution) in a frequency non-selective $(4 \times 4)$ MIMO system 


\section{Chapter 3}

\section{Signal Processing in MIMO Systems}

\subsection{Singular Value Decomposition}

Singular Value Decomposition (SVD) is one of the basic and most important tools of numerical analysis. This mathematical technique is used in order to avoid the inter-antenna interferences transforming from coupled into uncoupled ones that better expose the relationship among the input-output data items. SVD is based on a theorem from linear algebra which discovers that a matrix $\mathbf{A}$ can be factored as

$$
\mathbf{A}=\mathbf{U} \cdot \boldsymbol{\Sigma} \cdot \mathbf{V}^{\mathrm{H}},
$$

where $\mathbf{U}$ is an $(\mu \times \mu)$ orthogonal matrix which columns are the eigenvalues of $\mathbf{A} \cdot \mathbf{A}^{\mathrm{H}}, \mathbf{V}$ is an $(\nu \times \nu)$ orthogonal matrix which columns are the eigenvalues of $\mathbf{A}^{\mathrm{H}} \cdot \mathbf{A}$, and $\boldsymbol{\Sigma}$ is an $(\mu \times \nu)$ diagonal matrix of the form

$$
\boldsymbol{\Sigma}=\left(\begin{array}{cccc}
\sqrt{\xi^{(1)}} & 0 & \cdots & 0 \\
0 & \sqrt{\xi^{(2)}} & \cdots & 0 \\
\vdots & \vdots & \ddots & \vdots \\
0 & 0 & \cdots & \sqrt{\xi^{\left(L_{\mathrm{a}}\right)}}
\end{array}\right)
$$


where $\sqrt{\xi^{(1)}}>\sqrt{\xi^{(2)}}>\ldots>\sqrt{\xi^{(\mu)}}>\ldots>\sqrt{\xi^{\left(L_{\mathrm{a}}\right)}}>0$ and $L_{\mathrm{a}} \leq \min \left(n_{\mathrm{R}}, n_{\mathrm{T}}\right)$. The parameters $\xi^{(\mu)}$, with $\mu=1, \ldots, L_{\mathrm{a}}$, are defined as the eigenvalues of $\mathbf{A}^{\mathrm{H}} \cdot \mathbf{A}$ and the $\sqrt{\xi^{(\mu)}}$ are called the singular values of $\mathbf{A}$.

The SVD is a popular technique for processing MIMO channels in order to remove inter-antenna interferences. By applying the SVD, the MIMO channel can be described as multiple independent SISO layers with different performances determined by the layer corresponding with the singular value and the noise. Given the received antennas are disturbed by the same noise power, the larger the particular layer singular value, the higher the SISO channel reliability (lower BER). The ideal situation is when all singular values are equal and all layers present the same performance. The presence of predominant (strong and specially weak) layers decreases the channel performance. Singular values' distribution strongly depends on the existence of antenna's correlation.

Simulations In order to describe the receiver-side antenna's correlation effect on the channel performance a $(4 \times 4)$ frequency non-selective MIMO system under weak and strong correlation effect is simulated. Figure 3-1, Figure 3-2 and Figure 3-3 show the probability density function $(\mathrm{PDF})$ of the comparison between the smallest $\sqrt{\xi^{(4)}}$ and the highest $\sqrt{\xi^{(1)}}$ layer specific singular value for weak (CM-1) and strong (CM-2) correlation frequency non-selective $(4 \times 4)$ MIMO channels with linear and non-linear spacial distribution, both listed on Table 2.1 and Table 2.2.

From Figure 3-1 is observed that the distance between both layers increases as the correlation does. This means that the singular value ratio gets larger, and due to that the probability of having predominant layers increases affecting negatively to the channel performance.

A similar conclusion can be obtained by analysing the PDF curves from Figure 3-2 which shows that the impact of the correlation in a non-linear spacial distribution for weak correlation (CM-1) is even higher than in a linear spacial distribution.

Finally, in Figure 3-3 both spatial distributions are compared where it is noticed that the better performance for linear distribution (solid line) due to non-linear dis- 
tribution (dotted line) increases the probability of having layers with larger values more than linear distribution. 


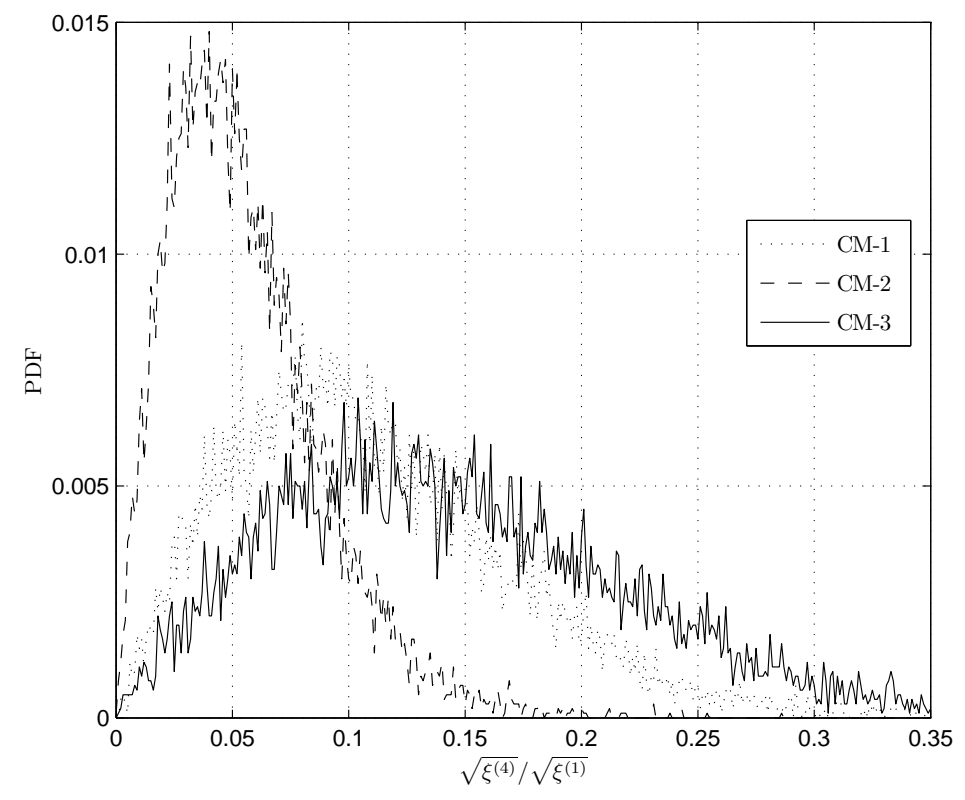

Figure 3-1: PDF (probability density function) of the comparison between the smallest $\sqrt{\xi^{(4)}}$ and the largest singular value $\sqrt{\xi^{(1)}}$ with linear spatial distribution with weak (CM-1) and strong (CM-2) correlation, and both are compared with no correlation one as a reference $(\mathrm{CM}-3)$ in a frequency non-selective $(4 \times 4)$ MIMO system

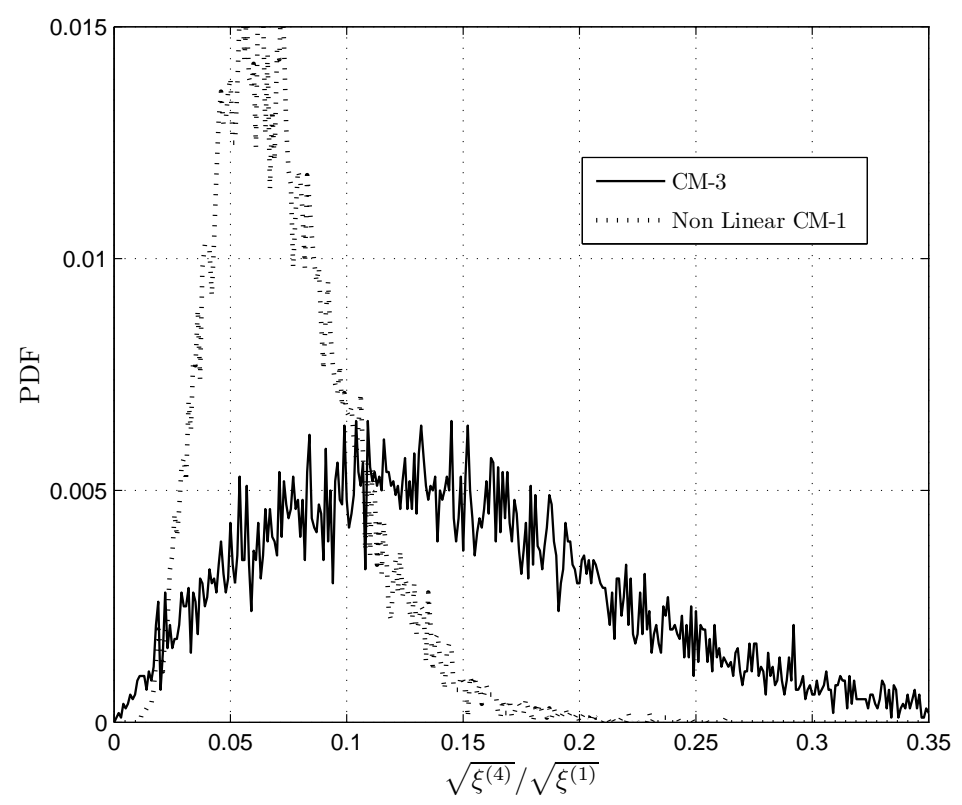

Figure 3-2: PDF (probability density function) of the comparison between the smallest $\sqrt{\xi^{(4)}}$ and the largest singular value $\sqrt{\xi^{(1)}}$ with non-linear spatial distribution with weak (CM-1) correlation compared with no correlation one as a reference (CM-3) in a frequency non-selective $(4 \times 4)$ MIMO system 


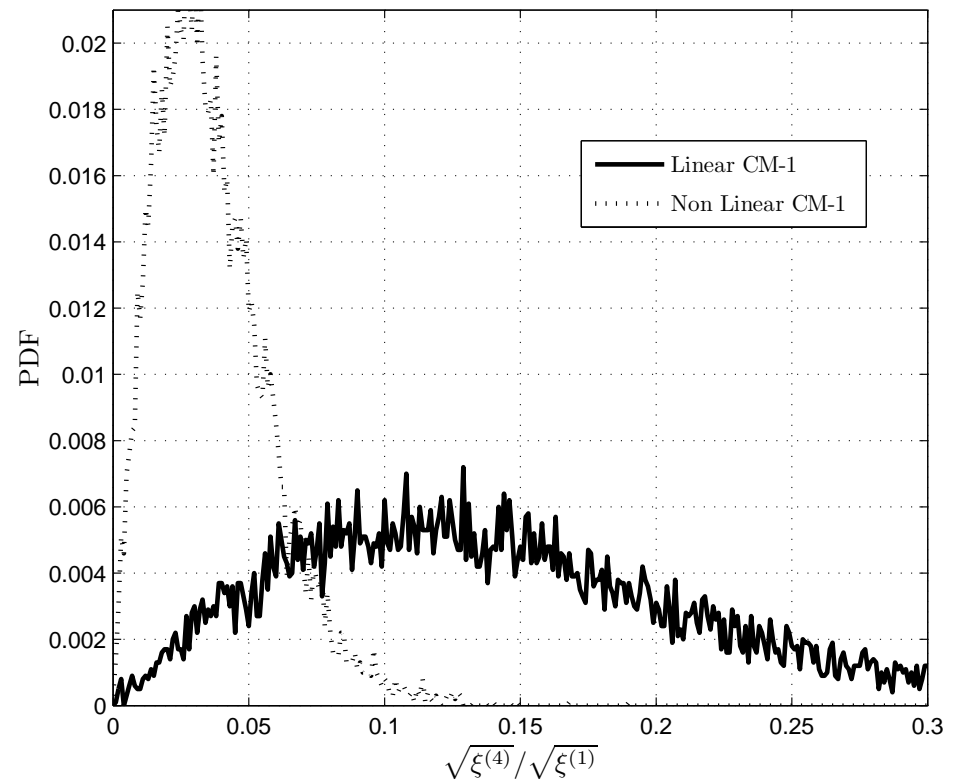

Figure 3-3: PDF (probability density function) of the comparison between the smallest $\sqrt{\xi^{(4)}}$ and the largest singular value $\sqrt{\xi^{(1)}}$ with linear and non-linear spatial distribution with weak $(\mathrm{CM}-1)$ correlation in a frequency non-selective $(4 \times 4) \mathrm{MIMO}$ system 


\subsection{SDM MIMO Model and Quality Criteria}

When considering a frequency non-selective SDM (space division multiplexing), MIMO link composed with $n_{\mathrm{T}}$ antennas in transmission and $n_{\mathrm{R}}$ in reception can be described as

$$
\mathbf{u}_{\mathrm{e}}=\mathbf{H} \cdot \mathbf{u}_{\mathrm{s}}+\mathbf{n}
$$

where $\mathbf{u}_{\mathrm{e}}$ corresponds to the $\left(n_{\mathrm{R}} \times 1\right)$ received vector, $\mathbf{H}$ is the $\left(n_{\mathrm{R}} \times n_{\mathrm{T}}\right)$ channel matrix, $\mathbf{u}_{\mathrm{s}}$ is the $\left(n_{\mathrm{T}} \times 1\right)$ transmitted vector and $\mathbf{n}$ is the Additive White Gaussian Noise (AWGN) vector. Furthermore, it is assumed that the coefficients of the $\left(n_{\mathrm{R}} \times\right.$ $n_{\mathrm{T}}$ ) channel matrix $\mathbf{H}$ are independently Rayleigh distributed with equal variance and that the number of transmit antennas $n_{\mathrm{T}}$ equals the number of receive antennas $n_{\mathrm{R}} \cdot$

Singular Value Decomposition (SVD) is well-established in MIMO signal processing where the whole MIMO channel is transferred into a number of weighted SISO channels. The unequal weighting of the SISO channels has led to intensive research to reduce the complexity of the required bit-and power-allocation techniques [40], [16], [7]. In order to avoid the inter-antenna interferences, Singular Value Decomposition (SVD) is used to transform the MIMO channel into independent layers. By applying the SVD technique to the MIMO channel matrix $\mathbf{H}$, it is possible to rewrite the channel matrix as $\mathbf{H}=\mathbf{S} \cdot \mathbf{V} \cdot \mathbf{D}^{\mathrm{H}}$, where $\mathbf{S}$ and $\mathbf{D}^{\mathrm{H}}$ are unitary matrices and $\mathbf{V}$ is a real-valued diagonal matrix of the positive square roots of the eigenvalues of the matrix $\mathbf{H}^{\mathrm{H}} \mathbf{H}$ sorted in descending order. By using the SVD technique in pre- and post processing, the MIMO channel can be described as multiple independent SISO layers with different gains given by the singular values in $\mathbf{V}$,

$$
\mathbf{y}=\mathbf{S}^{\mathrm{H}} \cdot \mathbf{u}_{\mathrm{e}}=\mathbf{S}^{\mathrm{H}}\left(\mathbf{H} \cdot \mathbf{D} \cdot \mathbf{u}_{\mathrm{s}}+\mathbf{n}\right)=\mathbf{V} \cdot \mathbf{u}_{\mathrm{s}}+\mathbf{w},
$$

where $\mathbf{H} \cdot \mathbf{D} \cdot \mathbf{u}_{\mathrm{s}}$ is the pre-processed transmit data vector and $\mathbf{S}^{\mathrm{H}} \cdot \mathbf{u}$ is the postprocessed data vector at the receiver side. 
According to the Chapter 1, the MIMO channel model with $n_{\mathrm{T}}$ transmit and $n_{\mathrm{R}}$ receive antennas for Rayleigh flat-fading like channels results in

$$
\operatorname{vec}(\mathbf{H})=\mathbf{R}_{\mathrm{HH}}^{\left(\frac{1}{2}\right)} \cdot \operatorname{vec}(\mathbf{G})
$$

where $\mathbf{G}$ is an $\left(n_{\mathrm{R}} \times n_{\mathrm{T}}\right)$ uncorrelated channel matrix with independent and identically distributed (i.i.d) complex Rayleigh distributed elements and $\operatorname{vec}(\cdot)$ the operator which stacks the matrix $\mathbf{G}$ into a vector column-wise. The matrix $\mathbf{R}_{\mathrm{HH}}$ describes the overall correlation and is described as

$$
\mathbf{R}_{\mathrm{HH}}=\mathbf{R}_{\mathrm{TX}} \otimes \mathbf{R}_{\mathrm{RX}}
$$

where $\otimes$ represents the Kronecker product.

The required measure for the end-to-end wireless communication system performance is given in terms of the bit-error-rate, which quantifies the reliability of the entire radio system from input bits to output bits, including the electronics devices, antennas configurations and signal paths effects between the transmit and receive side. A BER definition could be like this

$$
\mathrm{BER}=\frac{\text { Errors }}{\text { Total number of bits }} .
$$

The communication system works properly while this ratio is so small as to be insignificant for the quality of the whole communication system. It becomes significant when it is required to maintain a sufficient signal-to-noise ratio (SNR) in the presence of imperfect transmission mechanisms in a variable and unstable propagation medium.

\subsubsection{BER Performance in MIMO Systems}

BER probability definition in a MIMO system is presented by the next expression 


$$
P_{\mathrm{BER}}=\frac{b_{\mathrm{e}}^{(1)}+b_{\mathrm{e}}^{(2)}+\cdots+b_{\mathrm{e}}^{\left(L_{\mathrm{a}}\right)}}{b^{(1)}+b^{(2)}+\cdots+b^{\left(L_{\mathrm{a}}\right)}},
$$

where $b_{\mathrm{e}}^{\left(L_{\mathrm{a}}\right)}$ is the number of error bits in the layer $L_{\mathrm{a}}$ and $b^{\left(L_{\mathrm{a}}\right)}$ the transmitted number of bits modulated in $L_{\mathrm{a}}$ layer.

The BER probability formula depending on the index modulation can be expressed as

$$
P_{\mathrm{BER}}=\frac{b_{\mathrm{e}}^{(1)}+b_{\mathrm{e}}^{(2)}+\ldots+b_{\mathrm{e}}^{\left(L_{\mathrm{a}}\right)}}{b \cdot\left(\log _{2}\left(M_{1}\right)+\log _{2}\left(M_{2}\right)+\ldots+\log _{2}\left(M_{L_{\mathrm{a}}}\right)\right)},
$$

splitting the previous expression

$$
\begin{gathered}
P_{\mathrm{BER}}=\frac{b_{\mathrm{e}}^{(1)}}{b \cdot\left(\log _{2}\left(M_{1}\right)+\log _{2}\left(M_{2}\right)+\ldots+\log _{2}\left(M_{\mathrm{L}_{\mathrm{a}}}\right)\right)}+ \\
+\frac{b_{\mathrm{e}}^{(2)}}{b \cdot\left(\log _{2}\left(M_{1}\right)+\log _{2}\left(M_{2}\right)+\ldots+\log _{2}\left(M_{L_{\mathrm{a}}}\right)\right)}+ \\
+\cdots+\frac{n_{\mathrm{e}}^{\left(L_{\mathrm{a}}\right)}}{b \cdot\left(\log _{2}\left(M_{1}\right)+\log _{2}\left(M_{2}\right)+\ldots+\log _{2}\left(M_{L_{\mathrm{a}}}\right)\right)} .
\end{gathered}
$$

If the bit probability per layer $P_{\mathrm{b}}^{(\mu)}, \mu=1, \ldots, L_{\mathrm{a}}$, is defined as

$$
P_{\mathrm{b}}^{(\mu)}=\frac{b_{\mathrm{e}}^{(\mu)}}{b \cdot \log _{2}\left(M_{\mu}\right)},
$$

the $P_{\mathrm{BER}}$ is described with the following expression

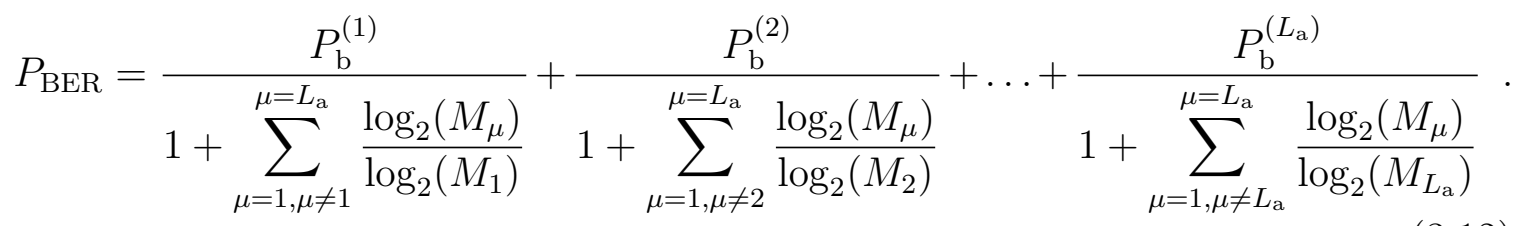

Finally, the overall MIMO bit-error rate expression results in 


$$
P_{\mathrm{BER}}=\frac{1}{\sum_{\mu=1}^{L_{\mathrm{a}}} \log _{2}\left(M_{\mu}\right)} \cdot \sum_{\mu=1}^{L_{\mathrm{a}}} P_{\mathrm{b}}^{(\mu)} \cdot \log _{2}\left(M_{\mu}\right)
$$

Taking into account the $M$-QAM modulation, the $P_{\mathrm{BER}}$ per MIMO layer can be expressed depending on the signal-to-noise ratio, $\varrho=\frac{U_{\mathrm{A}}^{2}}{U_{\mathrm{R}}^{2}}$, by

$$
P_{\mathrm{BER}}=\frac{2}{\log _{2}(M)} \cdot\left(1-\frac{1}{\sqrt{M}}\right) \cdot \operatorname{erfc}\left(\sqrt{\frac{\varrho}{2}}\right),
$$

where $U_{\mathrm{A}}$ corresponds with the half vertical eye opening and $U_{\mathrm{R}}$ with the noise power per quadrature component. [2].

The half-vertical eye-opening per MIMO active layer $\mu$ and time slot $k$ results in

$$
U_{\mathrm{A}}^{(\mu, k)}=\sqrt{\xi^{(\mu, k)}} \cdot U_{\mathrm{s}}^{(\mu)} .
$$

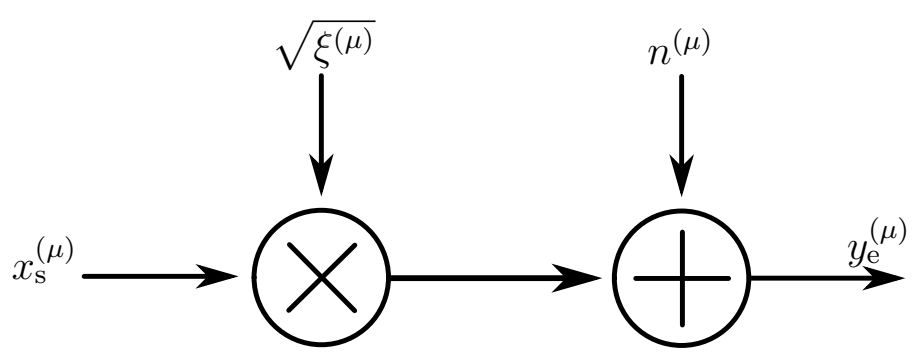

Figure 3-4: Layer-specific system model

Applying the system model per MIMO layer described in Figure 3-4, the $P_{\mathrm{BER}}^{(\mu)}$ becomes

$$
P_{\mathrm{BER}}^{(\mu, k)}=\frac{2}{\log _{2}\left(M_{\mu}\right)} \cdot\left(1-\frac{1}{\sqrt{M_{\mu}}}\right) \cdot \operatorname{erfc}\left(\sqrt{\frac{\xi^{(\mu, k)} \cdot\left(U_{\mathrm{s}}^{(\mu)}\right)^{2}}{2 \cdot U_{\mathrm{R}}^{2}}}\right) .
$$

Taking into account different QAM constellation sizes per active layer, the transmit power uniformly distributed over the MIMO layers $P_{\mathrm{s}}^{(\mu)}$ corresponds with 


$$
P_{\mathrm{s}}^{(\mu)}=\frac{P_{\mathrm{s}}}{L_{\mathrm{a}}}=\frac{2}{3} \cdot\left(U_{\mathrm{s}}^{(\mu)}\right)^{2} \cdot\left(M_{\mu}-1\right)
$$

where the overall transmit power $P_{\mathrm{s}}$ is fixed and uniformly distributed over the active layers

$$
P_{\mathrm{s}}=\sum_{\mu=1}^{L_{\mathrm{a}}} P_{\mathrm{s}}^{(\mu)}
$$

Thus, combining $P_{\mathrm{s}}^{(\mu)}$ with $P_{\mathrm{BER}}^{(\mu, k)}$ results

$$
P_{\mathrm{BER}}^{(\mu, k)}=\frac{2\left(1-\frac{1}{\sqrt{M_{\mu}}}\right)}{\log _{2}\left(M_{\mu}\right)} \cdot \operatorname{erfc}\left(\sqrt{\frac{3 \cdot \xi^{(\mu, k)}}{4 \cdot\left(M_{\mu}-1\right) \cdot U_{\mathrm{R}}^{2}} \cdot \frac{P_{\mathrm{s}}}{L_{\mathrm{a}}}}\right) .
$$

The resulting bit-error probability assuming different QAM constellation sizes per layer is obtained as

$$
P_{\mathrm{BER}}^{(k)}=\frac{2}{\sum_{\mu=1}^{L_{\mathrm{a}}} \log _{2}\left(M_{\mu}\right)} \cdot \sum_{\mu=1}^{L_{\mathrm{a}}}\left(1-\frac{1}{\sqrt{M_{\mu}}}\right) \cdot \operatorname{erfc}\left(\sqrt{\frac{3 \cdot \xi^{(\mu, k)}}{4 \cdot\left(M_{\mu}-1\right) \cdot U_{\mathrm{R}}^{2}} \cdot \frac{P_{\mathrm{s}}}{L_{\mathrm{a}}}}\right)
$$

Simulations In this section, BER curves are obtained from the different QAM transmission schemas listed in Table 3.1 in combination with the appropriate channel characteristic performance from the weak and strong correlated $(4 \times 4)$ MIMO system. Assuming these predefined QAM constellation sizes, a fixed data rate can be guaranteed for each data block.

The resulting BER curves for different QAM constellation sizes and MIMO configurations are depicted in Figure 3-5 and Figure 3-6 when transmitting at a bandwidth efficiency of 8 bit/s/Hz. Figure 3-5 shows the QAM transmission modes described in the Table 3.1 for a weak correlated scenario, meanwhile Figure 3-6 shows the strong correlated respectively (dotted lines). Hence, it turns out that not all MIMO layers have to be activated in order to achieve the best BER performance. More explicitly, the aim is to achieve a specific combination of the QAM mode and the number 
Table 3.1: Investigated QAM transmission modes

\begin{tabular}{lcccc}
\hline Throughput & layer 1 & layer 2 & layer 3 & layer 4 \\
\hline $8 \mathrm{bit} / \mathrm{s} / \mathrm{Hz}$ & 256 & 0 & 0 & 0 \\
$8 \mathrm{bit} / \mathrm{s} / \mathrm{Hz}$ & 64 & 4 & 0 & 0 \\
$8 \mathrm{bit} / \mathrm{s} / \mathrm{Hz}$ & 16 & 16 & 0 & 0 \\
$8 \mathrm{bit} / \mathrm{s} / \mathrm{Hz}$ & 16 & 4 & 4 & 0 \\
$8 \mathrm{bit} / \mathrm{s} / \mathrm{Hz}$ & 4 & 4 & 4 & 4 \\
\hline
\end{tabular}

of MIMO layers that presents the best possible BER performance at a given fixed $\mathrm{bit} / \mathrm{s} / \mathrm{Hz}$ bandwidth efficiency. 


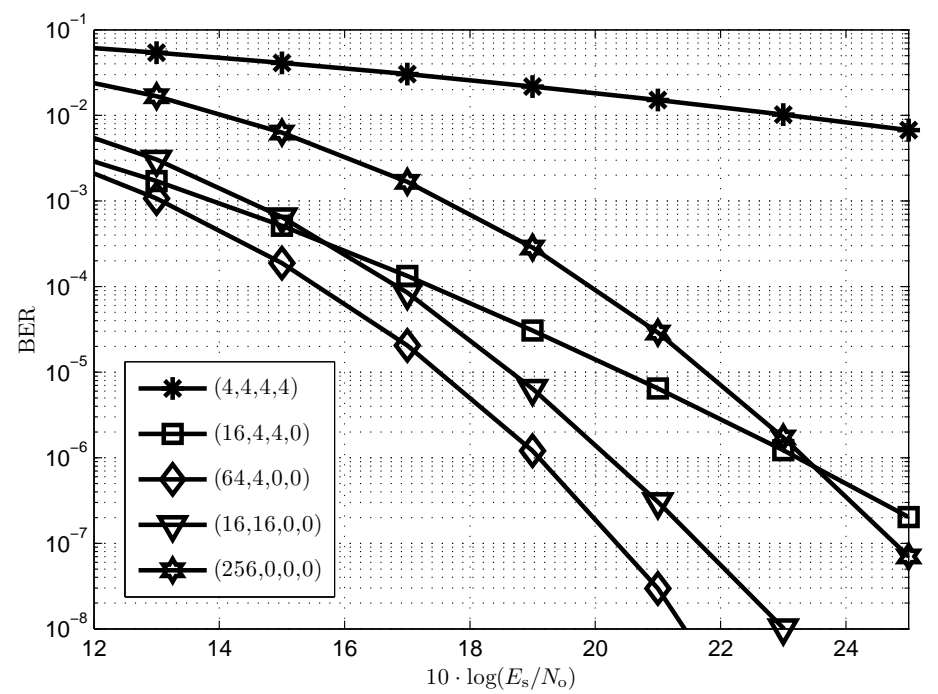

Figure 3-5: Bit-error probability weakly correlated with linear spatial distribution when using the transmission modes introduced in Table 3.1 and transmitting 8 $\mathrm{bit} / \mathrm{s} / \mathrm{Hz}$

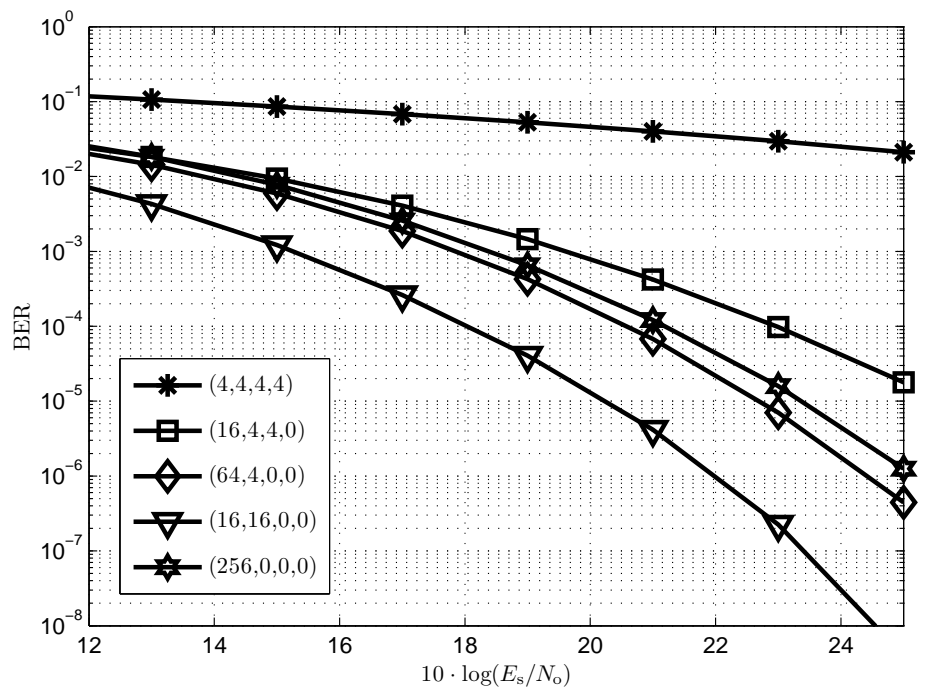

Figure 3-6: Bit-error probability strongly correlated with linear spatial distribution when using the transmission modes introduced in Table 3.1 and transmitting $8 \mathrm{bit} / \mathrm{s} / \mathrm{Hz}$ 


\subsection{Power Allocation}

In the previous sections, it has been assumed that the transmit power is uniformly distributed among the MIMO active layers. However it is possible to implement different techniques which allow adapting the power transmitted per every MIMO communication link depending on the channel performance. In that case, it should be firstly required to try to optimize the transmission power as much as possible. Adaptive bit and power allocation algorithms, which can significantly improve the MIMO system performance, can be divided into two groups according to their performance: optimal and suboptimal algorithms.

First, this section analyse an optimized scheme with fixed transmission modes per time slot [10]. Furthermore, the proposed algorithm performance has been compared to already developed strategies (equal-SNR power allocation and water-filling techniques) [9]. The novel contribution of this research is the proposal of a new iterative bit and power allocation (IBPA) approach per MIMO layer based on unequal power distribution per MIMO active layer.

Due to bit and power allocation techniques it is possible to use wireless channel in an optimal way, e.g., minimizing the BER performance at a fixed data rate under the constraint of a limited total MIMO transmit power. In general, the BER performance regarding the channel quality is affected by both the layer-specific weighting factors $\sqrt{\xi^{(\mu, k)}}$ and the QAM-constellation sizes $M_{\mu}$. Assuming a fixed data rate regardless the channel quality the resulting layer-specific QAM constellations for a fixed data throughput is displayed in Figure 3-7.

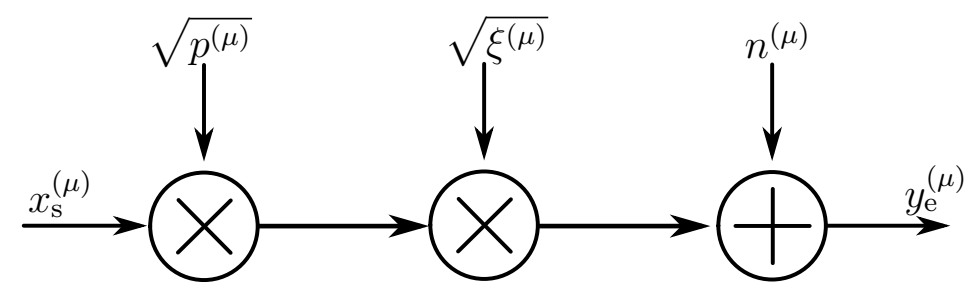

Figure 3-7: Layer-specific system model including power allocation parameter

The layer-specific power allocation factor $\sqrt{p^{(\mu, k)}}$, with $\mu=1, \ldots, L_{\mathrm{a}}$ and per 
symbol block $k$, adjusts the half-vertical eye opening according to

$$
U_{\mathrm{A} \text { PA }}^{(\mu, k)}=\sqrt{p^{(\mu, k)}} \cdot \sqrt{\xi^{(\mu, k)}} \cdot U_{\mathrm{s}}^{(\mu)}
$$

where $U_{\mathrm{s}}^{(\mu)}$ represents the half-level transmit amplitude assuming $M_{\mu}$-ary QAM modulation and $\sqrt{\xi^{(\mu, k)}}$ represents the singular values of the channel matrix $\mathbf{H}$.

The power allocation factor affects the layer-specific transmit power

$$
P_{\mathrm{SPA}}^{(\mu, k)}=p^{(\mu, k)} \cdot P_{\mathrm{s}}^{(\mu)}
$$

Taking all activated MIMO layers into account, the overall transmit power per symbol $k$ is obtained as

$$
P_{\mathrm{SPA}}^{(k)}=\sum_{\mu=1}^{L_{\mathrm{a}}} P_{\mathrm{SPA}}^{(\mu, k)}
$$

where $P_{\mathrm{SPA}}^{(k)}=P_{\mathrm{s}}^{(k)}$ and $L_{\mathrm{a}}$ represents the number of active layers.

The layer-specific bit-error probability assuming $M_{\mu}$-ary QAM modulation at the time slot $k$ changes to

$$
P_{\mathrm{BER}_{\mathrm{PA}}}^{(\mu, k)}=\frac{2}{\log _{2}\left(M_{\mu}\right)} \cdot\left(1-\frac{1}{\sqrt{M_{\mu}}}\right) \cdot \operatorname{erfc}\left(\frac{U_{\mathrm{A}_{\mathrm{PA}}}^{(\mu, k)}}{\sqrt{2} \cdot U_{\mathrm{R}}}\right) .
$$

In order to find an optimal set of PA parameters minimizing the overall BER the Lagrange multiplier method is employed. Using the Lagrange method, the cost function $J\left(p^{(1)}, \cdots, p^{\left(L_{\mathrm{a}}\right)}\right)$ may be expressed as [2]

$$
J(\cdots)=\frac{1}{\sum_{\nu=1}^{L_{\mathrm{a}}} \log _{2}\left(M_{\nu}\right)} \cdot \sum_{\mu=1}^{L_{\mathrm{a}}} \log _{2}\left(M_{\mu}\right) \cdot P_{\mathrm{BER}}^{(\mu, k)}+\lambda_{\mathrm{L}} \cdot B_{\mathrm{L}},
$$

where $\lambda_{\mathrm{L}}$ describes the Lagrange multiplier, and the parameter $B_{\mathrm{L}}$ denotes the Lagrange boundary condition

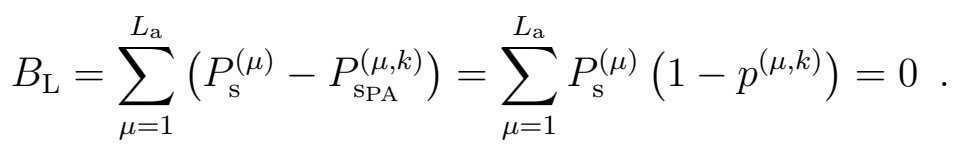


Without PA, a natural choice is to opt for a scheme that uniformly shares the transmit power along the number of activated MIMO layers, i.e. $P_{\mathrm{s}}^{(\mu)}=\frac{P_{\mathrm{s}}}{L_{\mathrm{a}}}$. In this case, the boundary condition simplifies to

$$
B_{\mathrm{L}}=\frac{P_{\mathrm{s}}}{L_{\mathrm{a}}} \sum_{\mu=1}^{L_{\mathrm{a}}}\left(1-p^{(\mu, k)}\right)=0
$$

Following this equation, the transmit power coefficients have to fulfil the following equation

$$
\sum_{\mu=1}^{L_{\mathrm{a}}} p^{(\mu, k)}=L_{\mathrm{a}}
$$

Differentiating the Lagrangian cost function $J\left(p^{(1, k)}, \cdots, p^{\left(L_{\mathrm{a}}, k\right)}\right)$ with respect to the $p^{(\mu, k)}$ and setting it to zero leads to the optimal set of PA parameters.

\subsubsection{Equal-SNR Power Allocation}

Since the optimal PA solution is notably computationally complex to be implemented, a suboptimal solution is analysed based on equal-SNR distribution. This PA technique concentrates on the SNR with power allocation per layer, $\varrho_{\mathrm{PA}}^{(\mu, k)}$, which is assumed to be equal for all MIMO layers.

Firstly, the available transmit power $P_{\mathrm{s}}$ is equally distributed along all the active layers getting $P_{\mathrm{s}}^{(\mu)}$ power per active layer before the power factor allocation.

$$
P_{\mathrm{s}}^{(\mu)}=\frac{2}{3} \cdot\left(U_{\mathrm{s}}^{(\mu)}\right)^{2} \cdot\left(M_{\mu}-1\right)
$$

from the previous equation the parameter $U_{\mathrm{s}}^{(\mu)}$ is resolved

$$
U_{\mathrm{s}}^{(\mu)}=\sqrt{\frac{3}{2} \cdot \frac{P_{\mathrm{s}}^{(\mu)}}{M_{\mu}-1}}
$$

Secondly, combining

$$
\varrho^{(\mu, k)}=\left(\frac{U_{\mathrm{A}}^{(\mu, k)}}{U_{\mathrm{R}}}\right)^{2}
$$


and

$$
U_{\mathrm{A}}^{(\mu, k)}=\sqrt{\xi^{(\mu, k)}} \cdot U_{\mathrm{s}}^{(\mu)}
$$

the parameter $\varrho^{(\mu, k)}$ is updated

$$
\varrho^{(\mu, k)}=\xi^{(\mu, k)} \cdot\left(\frac{U_{\mathrm{s}}^{(\mu)}}{U_{\mathrm{R}}}\right)^{2} .
$$

The power to be allocated to each activated and transmitted MIMO layer, $p^{(\mu, k)}$, can be calculated as follows [2]

$$
p^{(\mu, k)}=\frac{M_{\mu}-1}{\xi^{(\mu, k)}} \cdot\left(\frac{L_{\mathrm{a}}}{\sum_{\nu=1}^{L_{\mathrm{a}}} \frac{M_{\nu}-1}{\xi^{(\nu, k)}}}\right) .
$$

The parameter introduced in the system model is $\sqrt{p^{(\mu, k)}}$. Hence, once the power allocation parameters are obtained it is mandatory to recalculate the parameters introducing the power allocation coefficient per layer following the model shown in Figure 3-7.

$$
P_{\mathrm{SPA}}^{(\mu)}=\frac{2}{3} \cdot p^{(\mu, k)} \cdot\left(U_{\mathrm{s}}^{(\mu)}\right)^{2} \cdot\left(M_{\mu}-1\right)
$$

it is verified that

$$
\sum_{\mu=1}^{L_{\mathrm{a}}} P_{\mathrm{SPA}}^{(\mu)}=1
$$

and, finally, $\varrho_{\mathrm{PA}}^{(\mu, k)}$ is defined as

$$
\varrho_{\mathrm{PA}}^{(\mu, k)}=p^{(\mu, k)} \cdot \xi^{(\mu, k)} \cdot\left(\frac{U_{\mathrm{s}}^{(\mu)}}{U_{\mathrm{R}}}\right)^{2}
$$

The next step is to obtain the new BER curves with the power allocations' parameters but, firstly, the BER equation per layer is needed. 


$$
P_{\mathrm{BER}_{\mathrm{PA}}}^{(\mu, k)}=\frac{2\left(1-\frac{1}{\sqrt{M_{\mu}}}\right)}{\log _{2}\left(M_{\mu}\right)} \cdot \operatorname{erfc}\left(\sqrt{\frac{3 \cdot p^{(\mu, k)} \cdot \xi^{(\mu, k)}}{4 \cdot\left(M_{\mu}-1\right) \cdot U_{\mathrm{R}}^{2}} \cdot P_{\mathrm{SPA}}^{(\mu)}}\right),
$$

at the end the general power allocation bit-error probability expression is obtained

$$
P_{\mathrm{BER}_{\mathrm{PA}}}^{(k)}=\frac{2}{\sum_{\mu=1}^{L_{\mathrm{a}}} \log _{2}\left(M_{(\mu)}\right)} \sum_{\mu=1}^{L_{\mathrm{a}}} P_{\mathrm{BER}_{\mathrm{PA}}}^{(\mu, k)} .
$$

Simulations In order to study the effect of PA in $P_{\mathrm{BER}}$ in a MIMO communication system, two different time-invariant channel profiles are taken into account. These channel profiles are listed in Table 3.2. For comparison purposes, the channel profile (CP-1) focuses on a low correlation channel whereas the channel profile (CP-2) introduces a high degree of correlation.

Table 3.2: Investigated channel profiles assuming a $(4 \times 4)$ MIMO system

\begin{tabular}{cccccc}
\hline Profile & $\sqrt{\xi^{(4)}} / \sqrt{\xi^{(1)}}$ & Layer 1 & Layer 2 & Layer 3 & Layer 4 \\
\hline $\mathrm{CP}-1$ & 0.125 & 1.7500 & 0.8750 & 0.4375 & 0.2188 \\
$\mathrm{CP}-2$ & 0.0371 & 1.9000 & 0.6333 & 0.2111 & 0.0704 \\
\hline
\end{tabular}

In general, the BER is affected by both the layer-specific weighting factors $\sqrt{\xi^{(\mu)}}$ and the QAM constellation sizes $M_{\mu}$. Assuming a fixed bandwidth efficiency of 8 bits/s/Hz, the layer-specific QAM constellations are listed in Table 3.3.

Table 3.3: Investigated PA-QAM transmission modes

\begin{tabular}{ccccc}
\hline Throughput & Layer 1 & Layer 2 & Layer 3 & Layer 4 \\
\hline $8 \mathrm{bit} / \mathrm{s} / \mathrm{Hz}$ & 64 & 4 & 0 & 0 \\
$8 \mathrm{bit} / \mathrm{s} / \mathrm{Hz}$ & 16 & 16 & 0 & 0 \\
$8 \mathrm{bit} / \mathrm{s} / \mathrm{Hz}$ & 16 & 4 & 4 & 0 \\
\hline
\end{tabular}

Analysing the channel profiles from Table 3.2, the resulting BER curves with 
and without suboptimal PA, and with low and high correlation are highlighted in Figure 3-8, Figure 3-9 and Figure 3-10. It is noted from BER curves that PA technique is efficient in combination with an appropriate transmission modes to minimize the overall BER performance. In all situations, activating all MIMO layers does not necessary lead to the best BERs.

Figure 3-8, Figure 3-9 and Figure 3-10 describe a $(4 \times 4)$ MIMO system when transmitting at a bandwidth efficiency of $8 \mathrm{bit} / \mathrm{s} / \mathrm{Hz}$. The difference between these three figures is related to the QAM transmission modes: $(4,4,4,4),(16,4,4,0)$ and $(16,16,0,0)$ respectively. In each figure a comparison between the investigated channels profiles (CP-1 versus CP-2) is done. Furthermore, over each channel profile, a comparison between BER with power allocation (optimum and suboptimum) and BER without power allocation is depicted.

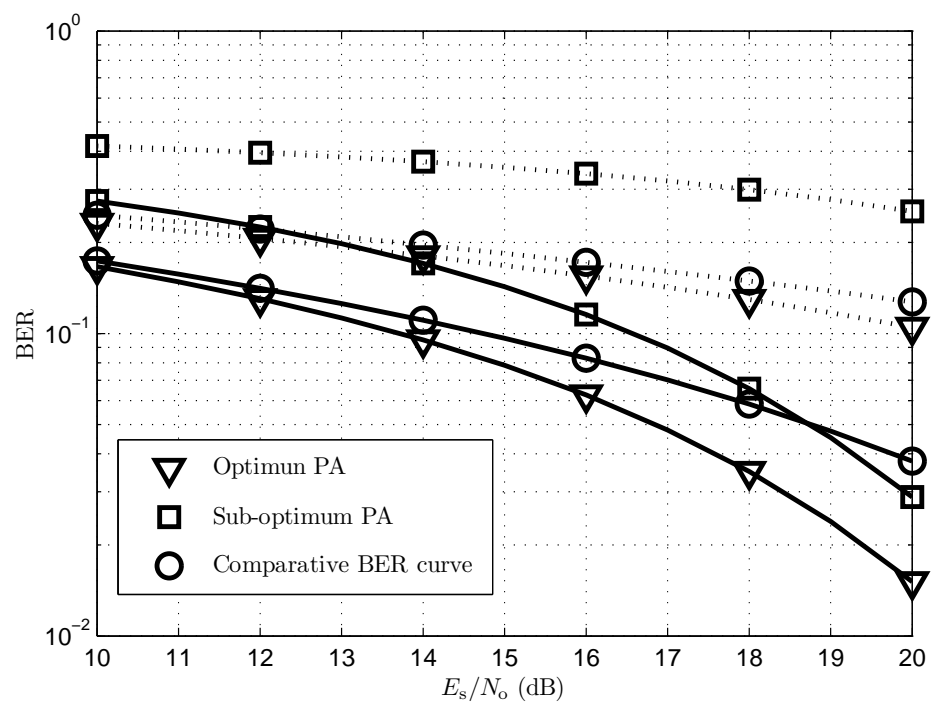

Figure 3-8: BER (Bit-error-rate) curves of $(4,4,4,4)$ QAM transmission mode comparing Optimum solution and Equal-SNR Power Allocation effect over (CP-1) (solid line) and (CP-2) (dotted line)

From these figures the power allocation improvement over BER MIMO performance is shown. For example, focusing on Figure 3-8 and fixing the BER value at $10^{-1}$ a BER improvement value of $1 \mathrm{~dB}$ approximately when comparing optimum power allocation solution with the transmission without power allocation in presence of low degree of correlation is extracted. In Figure 3-9, the PA strategy also improves 


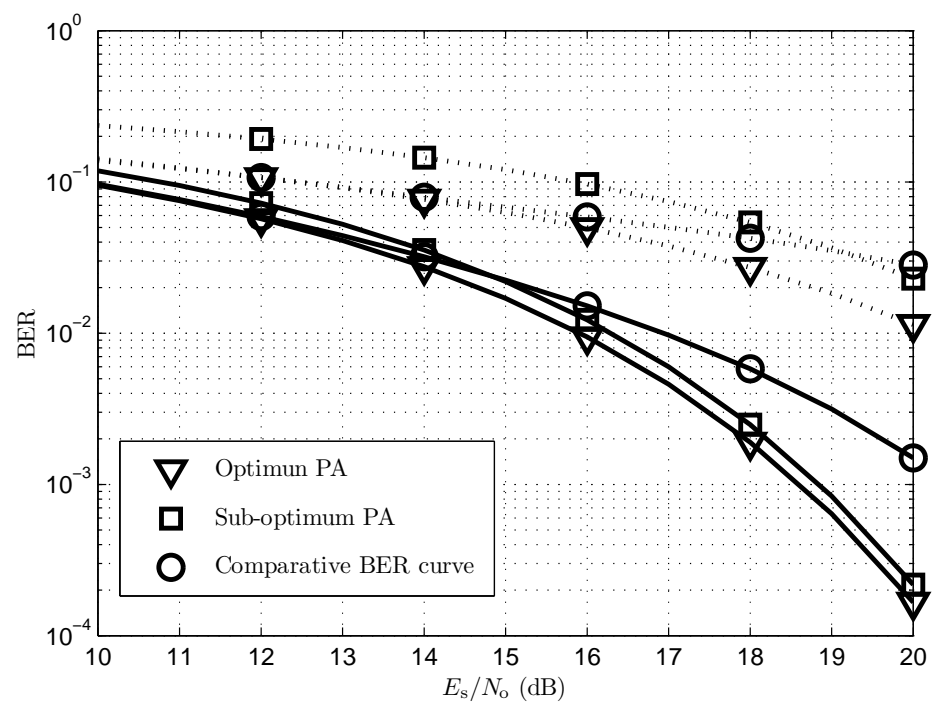

Figure 3-9: BER (Bit-error-rate) curves of $(16,4,4,0)$ QAM transmission mode comparing Equal-SNR Power Allocation effect over (CP-1) (solid line) and (CP-2) (dotted line)

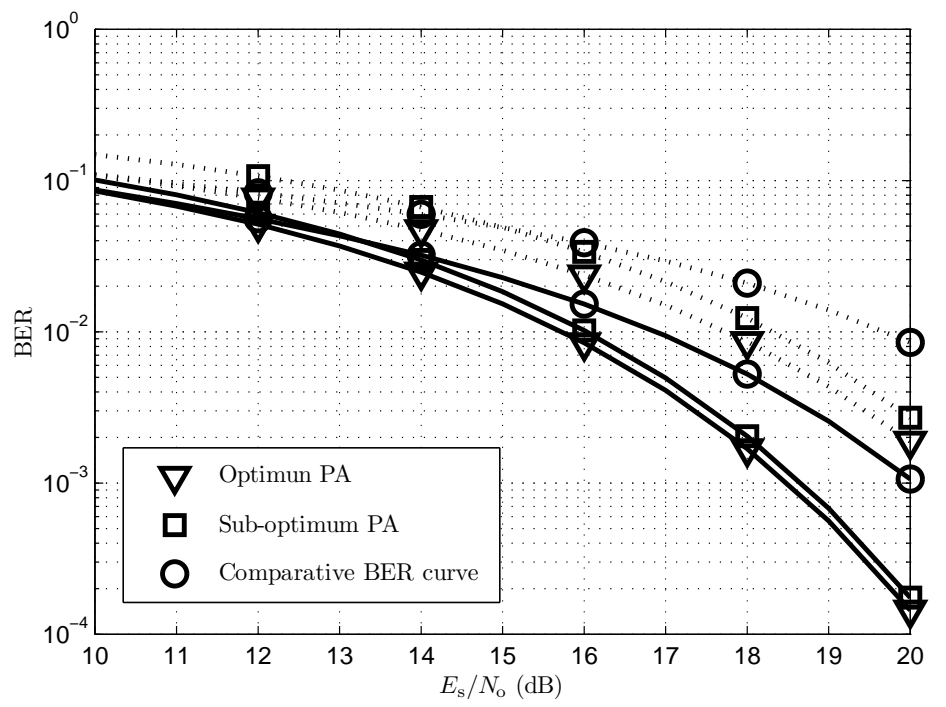

Figure 3-10: BER (Bit-error-rate) curves of $(16,16,0,0)$ QAM transmission mode comparing Equal-SNR Power Allocation effect over (CP-1) (solid line) and (CP-2) (dotted line) 
the $(16,4,4,0)$ BER curve in $2 \mathrm{~dB}$ for $(\mathrm{CP}-1)$ fixing the BER at $10^{-3}$. The BER improvement is also extended to $(16,16,0,0)$ transmission mode in Figure 3-10 with $2 \mathrm{~dB}$ for (CP-1). In Figures 3-9 and Figure 3-10, the suboptimal solution improves the BER performance in a similar quality than optimum solution does. Considering that the loss in the overall BER with the suboptimal equal-SNR PA criteria is quite acceptable compared to the optimal Lagrange-multiplier PA method, it is suitable to utilize a suboptimal equal-SNR PA technique in order to minimize BER performance due to the lower computational complexity and similar results. The BER improvements are also extended to the correlated channel profile (CP-2).

Pre-establishing the QAM constellation, depicted in Table 3.3, a fixed data rate is assured per data block ( 8 bit/s/Hz). Figure 3-13 shows the PDF of the transmission modes introduced in Table 3.3 and transmitting 8 bit/s/Hz over uncorrelated, weak and strong (channels profiles listed on Table 3.2) frequency non-selective $(4 \times 4)$ MIMO channels. This highlights how the correlation effect modifies the QAM transmission mode chosen, decreasing the number of active layers and increasing the transmission mode in the highest $\sqrt{\xi^{(1)}}$ layer specific singular value.

The resultant BER curves with fixed QAM constellation are depicted in Figure 311 and Figure 3-12 for the two different investigated channel profiles from Table 3.2, when transmitting at a bandwidth efficiency of $8 \mathrm{bis} / \mathrm{s} / \mathrm{Hz}$. Thus, it is highlighted that not all MIMO layers have to be activated in order to achieve the best BERs. The target is to find out the best combination of the QAM mode and the number of MIMO layers, which gives the best possible BER performance at $8 \mathrm{bit} / \mathrm{s} / \mathrm{Hz}$ bandwidth efficiency. Subsequently, PA techniques are applied for minimizing the bit-error probability. On the other hand, by comparing Figure 3-11 and Figure 3-12 the increasing BER due to antennas correlation is noted.

From these obtained BER curves the benefits of using power allocation strategies over MIMO systems are demonstrated with different improvement levels depending on the QAM transmission selected. 


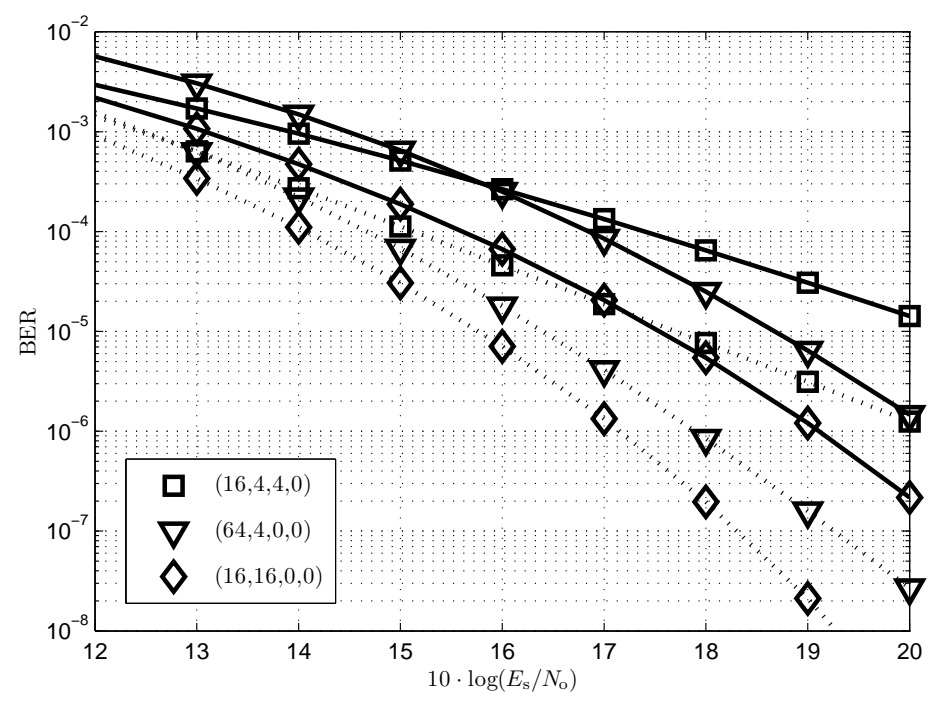

Figure 3-11: BER with equal-SNR PA (dotted line) and without PA (solid line) when using the transmission modes introduced in Table 3.3 and transmitting $8 \mathrm{bit} / \mathrm{s} / \mathrm{Hz}$ over frequency non-selective $(4 \times 4)$ MIMO channels with weak antenna correlation

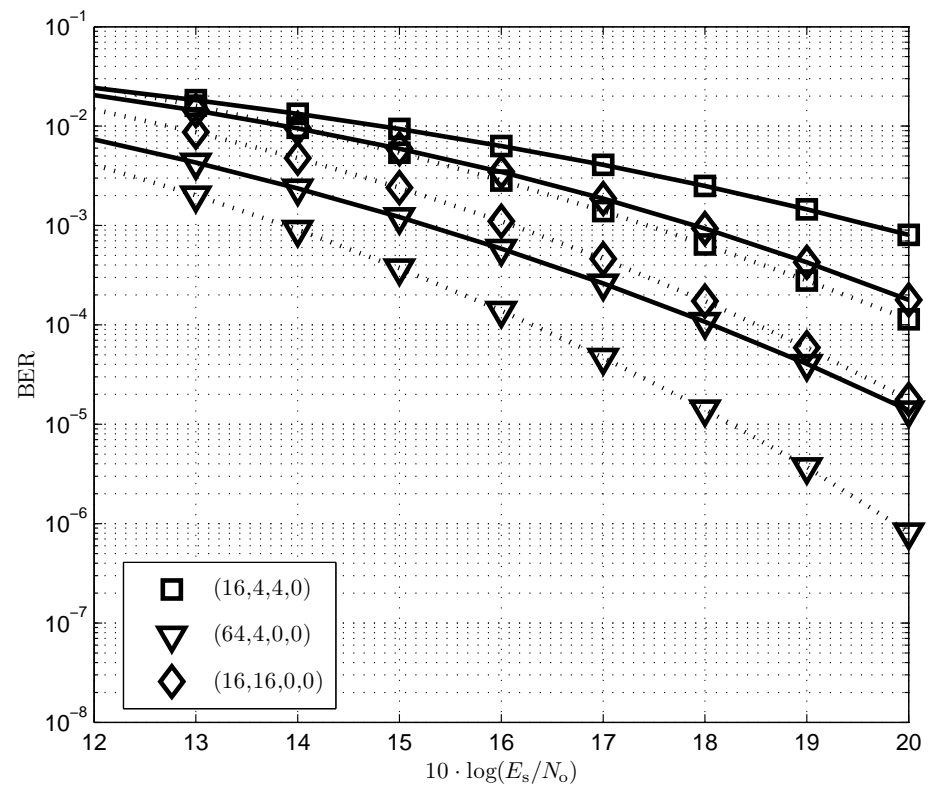

Figure 3-12: BER with equal-SNR PA (dotted line) and without PA (solid line) when using the transmission modes introduced in Table 3.3 and transmitting $8 \mathrm{bit} / \mathrm{s} / \mathrm{Hz}$ over frequency non-selective $(4 \times 4)$ MIMO channels with strong antenna correlation 


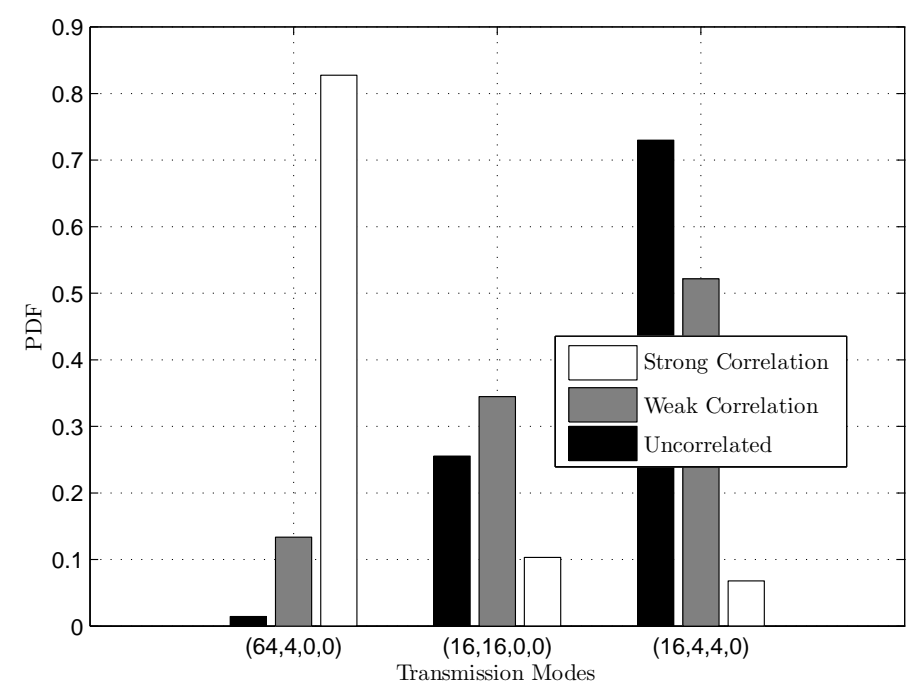

Figure 3-13: PDF (probability density function) of choosing different transmission modes when using the transmission modes introduced in Table 3.3 and transmitting $8 \mathrm{bit} / \mathrm{s} / \mathrm{Hz}$ over uncorrelated, weakly correlated (CM-1) and strongly correlated (CM2) frequency non-selective $(4 \times 4)$ MIMO channels

\subsubsection{Water-filling}

Water-filling is a power allocation technique first described by Shannon in the 1940s. This method, being given a number of parallel sub-channels with different attenuations, is able to find out the optimal distribution of transmission power that maximizes capacity for an arbitrary channel profile with different singular values per layer.

Water-filling technique is illustrated in Figure 3-14. It is required to imagine a number of connected vessels. At the bottom of each vessel there is a block of concrete with height that is proportional to the inverse SNR of the sub-channel that it being considered. Then take water, and pour it into the vessels; the amount of poured water is proportional to the total transmit power that is available. Due to the fact that the vessels are connected, the surface level of the water is guaranteed to be flat.

The layer-specific system model including the power parameter based on waterfilling resource allocation technique is highlighted in Figure 3-15.

The well-known water-filling technique which is virtually synonymous with adaptive modulation, tries to exclude the symbol positions having the smallest SNR. The channel condition on the $\mu$-th MIMO layer at the time $k$ is characterized by the $\mu$-th 


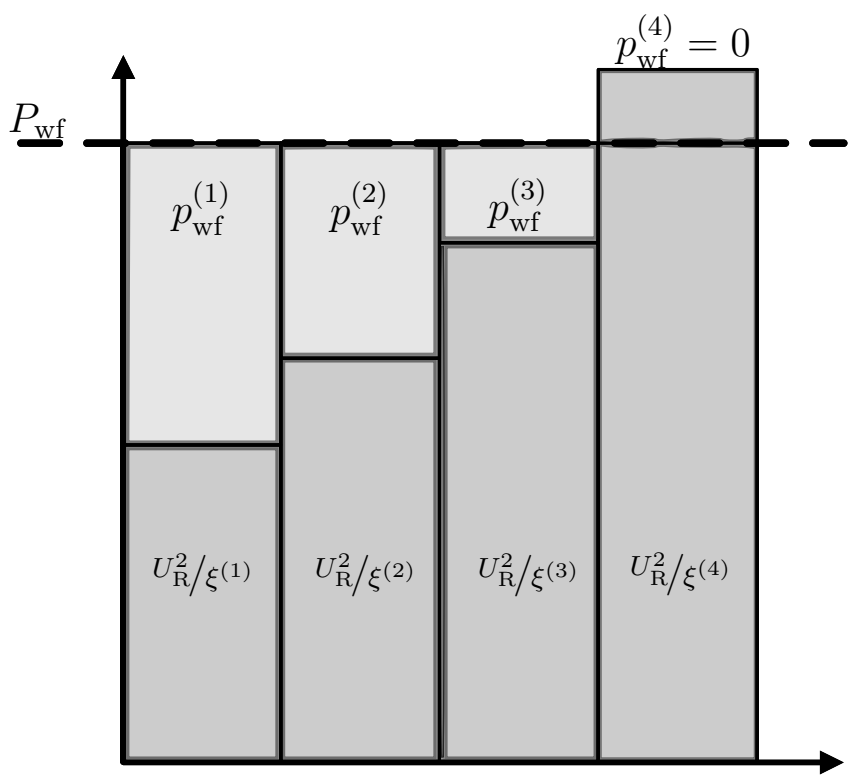

Figure 3-14: Water-filling diagram for a fixed time slot

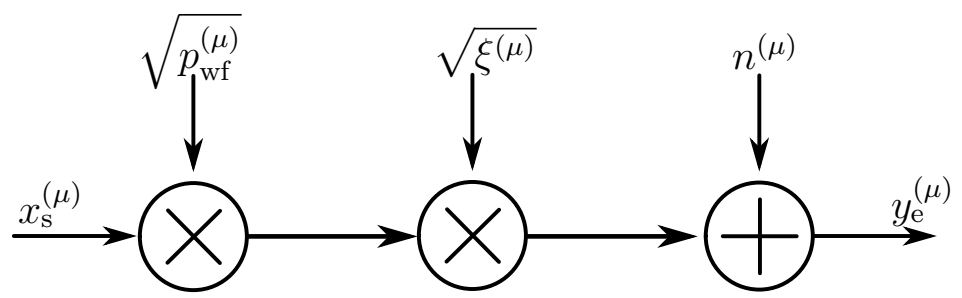

Figure 3-15: Layer-specific system model including power allocation parameter with water-filling technique

singular value $\sqrt{\xi^{(\mu, k)}}$ and hence the $\mu$-th MIMO layer is assigned the transmit power $P_{\mathrm{s}}^{(\mu, k)}$ according to the water-filling principle with

$$
P_{\mathrm{s}}^{(\mu, k)}=\left(P_{\mathrm{wf}}^{(k)}-\frac{U_{\mathrm{R}}^{2}}{\xi^{(\mu, k)}}\right)^{+}
$$

where

$$
(x)^{+}=\left\{\begin{array}{l}
x \text { if } x \geq 0 \\
0 \text { otherwise }
\end{array}\right.
$$

The parameter $P_{\mathrm{wf}}^{(k)}$ is determined so that is ensured 


$$
\sum_{\mu=1}^{L_{\mathrm{a}}} P_{\mathrm{s}}^{(\mu, k)}=P_{\mathrm{s}}^{(k)}
$$

where $P_{\mathrm{s}}^{(k)}$ is the total available transmit power. The parameter $P_{\mathrm{wf}}^{(k)}$ depends on the used number of MIMO layers $L_{\mathrm{a}}$ per transmitted symbol block $k$ and results in

$$
P_{\mathrm{wf}}^{(k)}=\frac{P_{\mathrm{s}}}{L_{\mathrm{a}}}+\frac{U_{\mathrm{R}}^{2}}{L_{\mathrm{a}}} \sum_{\mu=1}^{L_{\mathrm{a}}} \frac{1}{\xi(\mu, k)} .
$$

Using the half-vertical eye opening expression, $U_{\mathrm{A}}^{(\mu, k)}=\sqrt{\xi^{(\mu, k)}} \cdot U_{\mathrm{s} \mu}$, the SNR on each MIMO layer results with the parameter $P_{\mathrm{s}}^{(\mu, k)}$ in

$$
\varrho^{(\mu, k)}=\xi^{(\mu, k)} \cdot \frac{U_{\mathrm{s} \mu}^{2}}{U_{\mathrm{R}}^{2}} \equiv \varrho_{\mathrm{wf}},
$$

where $\mu=1,2, \ldots, L_{\mathrm{a}}$.

A natural choice is again to opt for a PA scheme, which results in an identical SNR for each MIMO channel output and each symbol. In this specific case, the average transmit power per MIMO layer, defined by $P_{\mathrm{s}}^{(\mu)}=\frac{2}{3} \cdot\left(U_{\mathrm{s}}^{(\mu)}\right)^{2} \cdot\left(M_{\mu}-1\right)$, results in

$$
P_{\mathrm{s}}^{(\mu, \mathrm{k})}=\frac{2}{3} \cdot \frac{\varrho_{\mathrm{wf}} \cdot U_{\mathrm{R}}^{2}}{\xi^{(\mu, k)}} \cdot\left(M^{(\mu, k)}-1\right)
$$

needed to transmit $\log _{2}\left(M^{(\mu, k)}\right)$ bits with the quality over the layer $\mu$ and the time slot $k$. Using this water-filling optimized averaged transmit power $P_{\mathrm{s}}^{(\mu, k)}$ per MIMO layer, the supported real-valued numbers of signal points per MIMO layer result in

$$
M^{(\mu, k)}=1+\frac{2}{3} \cdot \frac{\xi^{(\mu, k)}}{\varrho_{\mathrm{wf}}} \cdot \frac{P_{\mathrm{s}}^{(\mu, k)}}{U_{\mathrm{R}}^{2}} .
$$

Unfortunately, in order to be able to use QAM constellations, a round-off of the calculated ones is required. The choice of the next smallest power of two as a practically favourable number of signal points per MIMO layer leads to the following integer number of bits per MIMO layer and QAM symbol (the expression $\lfloor\cdot\rfloor$ delivers the next smallest integer value). 


$$
\log _{2}\left(\hat{M}^{(\mu, k)}\right)=\left\lfloor\log _{2}\left(M^{(\mu, k)}\right)\right\rfloor
$$

However, the rounding operation leads to a remaining power that can be used to adapt the half level amplitudes and becomes

$$
\hat{U}_{\mathrm{s}}^{(\mu, k)}=\sqrt{\frac{3}{2} \cdot \frac{P_{\mathrm{s}}^{(\mu, k)}}{\left(\hat{M}^{(\mu, k)}-1\right)}} .
$$

Assuming the equal-SNR on all active layers, the rounding operation now results in a MIMO specific SNR per layer

$$
\hat{\varrho}^{(\mu, k)}=\frac{\xi^{(\mu, k)} \cdot \hat{U}_{\mathrm{s}}^{(\mu, k)}}{U_{\mathrm{R}}^{2}} .
$$

As a consequence of the unequal SNR, different BERs per MIMO layer arise according to

$$
P_{\mathrm{BER}_{\mathrm{wf}}}^{(\mu, k)}=\frac{2}{\log _{2}\left(\hat{M}^{(\mu, k)}\right)} \cdot\left(1-\frac{1}{\sqrt{\hat{M}^{(\mu, k)}}}\right) \cdot \operatorname{erfc}\left(\sqrt{\frac{\hat{\varrho}^{(\mu, k)}}{2}}\right) .
$$

The general bit-error-rate expression extends to all active layers with the following expression

$$
P_{\mathrm{BER}_{\mathrm{wf}}}=\frac{2}{\sum_{\mu=1}^{L_{\mathrm{a}}} \log _{2}\left(M_{(\mu)}\right)} \sum_{\mu=1}^{L_{\mathrm{a}}} P_{\mathrm{BER}_{\mathrm{wf}}^{(\mu)}}
$$

In order to balance the different layer-specific bit-error probabilities the beforehand introduced PA scheme can be helpful. Since the transmit power is not uniformly distributed any longer, the power allocation parameters can be calculated according to

$$
p_{\mathrm{wf}}^{(\mu, k)}=\frac{1}{P_{\mathrm{s}}^{(\mu, k)}} \cdot \frac{\hat{M}^{(\mu, k)}-1}{\xi^{(\mu, k)}} \cdot \frac{1}{\sum_{\nu=1}^{L_{\mathrm{a}}} \frac{\left(\hat{M}^{(\nu, k)}-1\right)}{\xi^{(\nu, k)}}} .
$$


Simulations In order to study the improvement of using the water-filling power allocation technique over the bit-error probability, the two channel profiles listed in Table 2.1 for weak and strong correlation are taken into account for a frequency non-selective $(4 \times 4)$ MIMO communication system.

The resultant BER curves when using the water-filling principle with the optimized PA are depicted in Figure 3-16 for weak correlation effect and in Figure 3-17 for strong correlation when transmitting at an averaged bandwidth efficiency of 8 bit/s/Hz respectively. Therein, the minimum number of bits transmitted per MIMO layer and QAM symbol is restricted by 4 .

In order to compare the effects produced by water-filling power allocation technique over the global quality, both figures highlight a significantly better performance compared with the fixed QAM constellation sizes depicted in the previous Equal-SNR PA section.

The BER curves plotted in Figure 3-16 show the power allocation improvement by the water-filling algorithm over BER MIMO performance. For example, fixing the BER value at $10^{-7}$ extracts a BER improvement value close to $2 \mathrm{~dB}$ in presence of weak correlation. A similar conclusion can be obtained by analysing the BER curves from Figure 3-17 with approximately an improvement of $2 \mathrm{~dB}$ with strong correlation effect for fixing at $10^{-6}$ the BER value. 


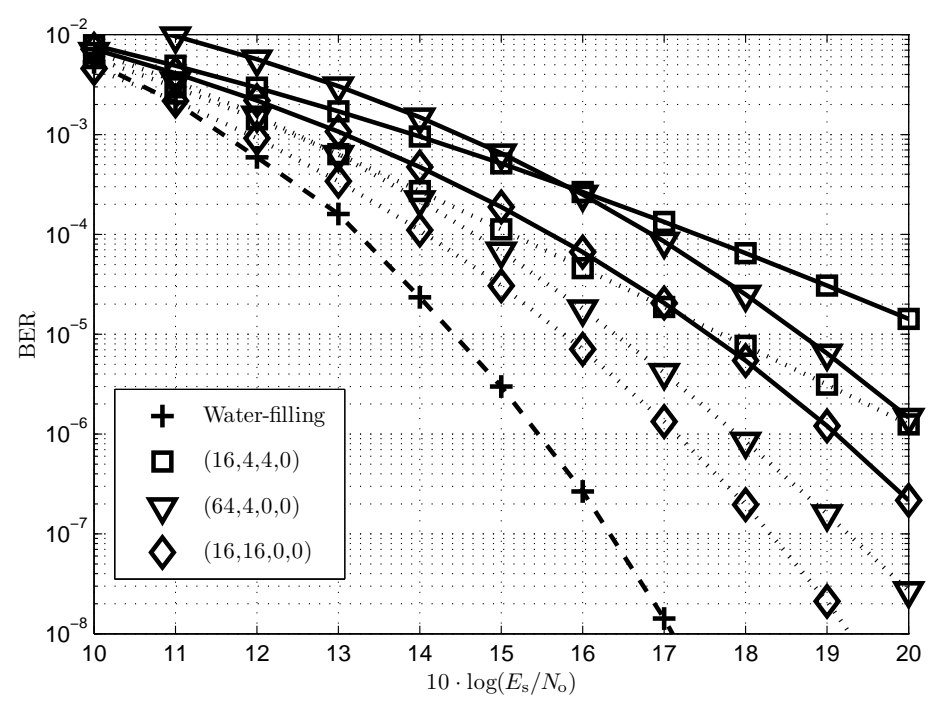

Figure 3-16: BER with Water-filling (dashed line) compared to BER curves with equal-SNR PA (dotted line) and without PA (solid line) when using the transmission modes introduced in Table 3.1 and transmitting $8 \mathrm{bit} / \mathrm{s} / \mathrm{Hz}$ over frequency nonselective $(4 \times 4)$ MIMO channels with weak antenna correlation

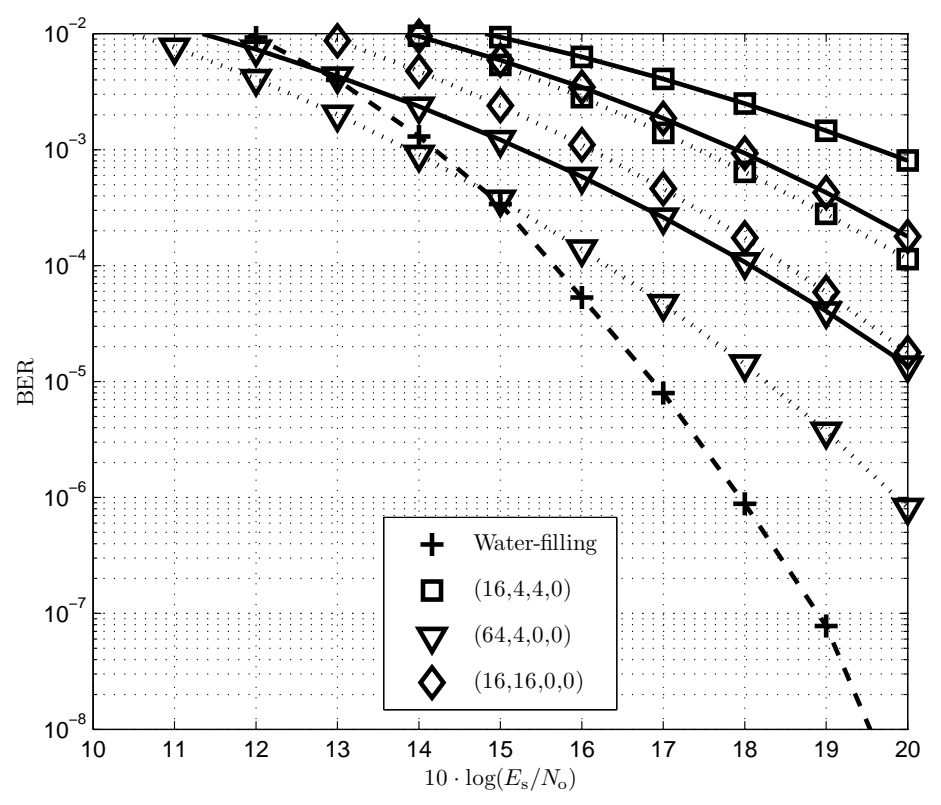

Figure 3-17: BER with Water-filling (dashed line) compared to BER curves with equal-SNR PA (dotted line) and without PA (solid line) when using the transmission modes introduced in Table 3.1 and transmitting 8 bit/s/Hz over frequency nonselective $(4 \times 4)$ MIMO channels with strong antenna correlation 


\subsubsection{Iterative Bit and Power Allocation}

Following the investigated fixed transmission modes, iterative bit and power allocation strategies seems to be the most challenging solutions when minimizing the overall BER. Hence, the efficiency of bit auctioning algorithms is investigated. Such solutions test the amount of transmit power needed to transmit a given number of bits per layer before allocating the bit to the layer and deciding for a constellation which requires the minimum power. For the iterative bit and PA solution investigated in this work, it is first tested in which layer the allocation of an extra bit requires the minimum transmit power taking all activated layers into account $\left(\mu=1,2, \ldots, L_{\mathrm{a}}\right)$.

In order to find out the best bit allocation performance per time slot, finding the appropriate QAM constellation with the minimum transmit power is required. For that, firstly, from equation (3.13) it is required to solve the SNR parameter resulting in

$$
\varrho_{\mathrm{IBPA}}^{(\mu, k)}=2 \cdot \text { inverfc }^{2}\left(\frac{P_{\mathrm{BER}}^{(\mu, k)} \cdot \log _{2}\left(M_{\mu}\right)}{2 \cdot\left(1-\frac{1}{\sqrt{M_{\mu}}}\right)}\right) .
$$

The half-level transmit amplitude on each MIMO layer results together with the signal-to-noise ratio, $\varrho=\left(\frac{U_{\mathrm{A}}^{(\mu, k)}}{U_{\mathrm{R}}}\right)^{2}$, and the half-vertical eye opening, $U_{\mathrm{A}}^{(\mu, k)}=$ $\sqrt{\xi^{(\mu, k)}} \cdot U_{\mathrm{s}}^{(\mu)}$, in

$$
\left(U_{\mathrm{s} \text { IBPA }}^{(\mu)}\right)^{2}=\frac{\varrho_{\mathrm{IBPA}}^{(\mu, k)}}{\xi^{(\mu, k)}} \cdot U_{\mathrm{R}}^{2} .
$$

Consequently, using (3.53) and (3.54), the transmit power per MIMO layer (3.17) can be obtained as

$$
P_{\mathrm{s} \mathrm{IBPA}}^{(\mu, k)}=\frac{4}{3} \cdot\left(M_{\mu}-1\right) \cdot \frac{U_{\mathrm{R}}^{2}}{\xi^{(\mu, k)}} \cdot \operatorname{inverfc}^{2}\left(\frac{P_{\mathrm{BER}}^{(\mu, k)} \cdot \log _{2}\left(M_{\mu}\right)}{2 \cdot\left(1-\frac{1}{\sqrt{M_{\mu}}}\right)}\right)
$$

With a given noise power per quadrature component $U_{\mathrm{R}}^{2}$, the layer-specific weighting $\xi^{(\mu, k)}$ and a reference bit-error rate $P_{\mathrm{BER}}^{(\mu, k)}$, the transmit power per layer can be 
calculated for a given $M_{\mu}$.

In the first approach, the proposed IBPA solution focuses on minimizing the overall BER at a fixed data rate. Figure 3-18a illustrates the working principle at a fixed data rate of 8 bit/s/Hz using channel (CP-2). According to Figure 3-18b, firstly, the bit-error rate level is fixed. Next, the bit allocation process is started with two bits. The QAM constellation is transmitted over the layer with the minimum power. Hence, layer $\mu=1$ and the $(4,0,0,0)$ transmission mode is selected. After that, as the overall transmit power $P_{\mathrm{s} \text { IBPA }}^{(k)}=\sum_{\mu=1}^{L_{\mathrm{a}}} P_{\mathrm{s}}^{(\mu, k)}$ is lower than one, another two bits are allocated. Resulting in the $(16,0,0,0)$ transmission mode depicted in the second row from Figure 3-18a. In the third row, as another two more bits are allocated, the $P_{\mathrm{s} \text { IBPA }}^{(k)}$ exceeds the power limitation so the BER level is increased to $10^{-6}$, being $(16,4,0,0)$ the updated QAM transmission mode. In the fourth row, the last two bits are allocated due to the $8 \mathrm{bit} / \mathrm{s} / \mathrm{Hz}$ limitation. In this last iteration, the BER level is increased to $10^{-3}$. This results in the $(64,4,0,0)$ transmission mode. As the available 8 bits have been allocated, the bit allocation process is finished.

After the bit allocation process, power allocation can be used to share the remaining power if $P_{\mathrm{S} \text { IBPA }}^{(\mu, k)}<1$ and to balance the bit error probabilities in the number of activated MIMO layers.

Since the transmit power is not uniformly distributed, the beforehand developed power allocation schemes cannot be used any longer, with $P_{\mathrm{s}}^{(\mu, k)} \neq \frac{P_{\mathrm{IBPA}}^{(k)}}{L_{\mathrm{a}}}$. In this case, the transmit power allocation parameter has to fulfil the following boundary condition

$$
P_{\mathrm{s}}^{(k)}=\sum_{\mu=1}^{L_{\mathrm{a}}} p_{\mathrm{IBPA}}^{(\mu, k)} \cdot P_{\mathrm{s} \mathrm{IBPA}}^{(\mu, k)}
$$

Again, a suboptimal power allocation which concentrates on the argument of the complementary error function can be found resulting in 


$$
\varrho_{\mathrm{IBPA}}^{(\mu, k)}=\xi^{(\mu, k)} \cdot p_{\mathrm{IBPA}}^{(\mu, k)} \cdot \frac{\left(U_{\mathrm{s} \mu}\right)^{2}}{U_{\mathrm{R}}^{2}},
$$

which is assumed to be equal for all activated MIMO layers per data block $k$, i.e., $\varrho_{\mathrm{IBPA}}^{(\mu, k)}=$ constant $\left(\mu=1,2, \ldots, L_{\mathrm{a}}\right)$. The new power parameter $p_{\mathrm{IBPA}}^{(\mu, k)}$ is calculated for each activated MIMO layer $\mu$ and transmitted data block $k$, taking into consideration the transmit power $P_{\mathrm{s} \text { IBPA }}^{(\mu, k)}$ per MIMO layer (3.55), the boundary condition (3.56) and the SNR per quadrature component (3.57). The new power parameter $p_{\text {IBPA }}^{(\mu, k)}$ can be calculated as follows

$$
p_{\mathrm{IBPA}}^{(\mu, k)}=\frac{1}{P_{\mathrm{S} \mathrm{IBPA}}^{(\mu, k)}} \cdot \frac{M_{\mu}-1}{\xi^{(\mu, k)}} \cdot \frac{1}{\sum_{\nu=1}^{L_{\mathrm{a}}} \frac{\left(M_{\nu}-1\right)}{\xi^{(\nu, k)}}} .
$$

Using the power parameter $p_{\mathrm{IBPA}}^{(\mu, k)}$ from equation (3.58) to be allocated in each transmitted MIMO symbol, the half vertical eye opening changes to

$$
U_{\mathrm{A} \text { IBPA }}^{(\mu, k)}=\sqrt{p_{\mathrm{IBPA}}^{(\mu, k)}} \cdot \sqrt{\xi^{(\mu, k)}} \cdot U_{\mathrm{s} \text { IBPA }}^{(\mu)}
$$

modifying the MIMO SNR per layer

$$
\varrho_{\mathrm{IBPA}}^{(\mu, k)}=p_{\mathrm{IBPA}}^{(\mu, k)} \cdot \xi^{(\mu, k)} \cdot \frac{\left(U_{\mathrm{s} \mathrm{IBPA}}^{(\mu)}\right)^{2}}{U_{\mathrm{R}}^{2}} .
$$

With the obtained transmit power per MIMO layer $\mu$ and the symbol block $k$, the half level transmit amplitude results in

$$
U_{\mathrm{s} \text { IBPA }}^{(\mu)}=\sqrt{\frac{3}{2} \cdot \frac{P_{\mathrm{s} \text { IBPA }}^{(\mu, k)}}{\left(M_{\mu}-1\right)}}
$$

Consequently, using (3.60) and (3.61), the bit-error rate per MIMO layer $P_{\mathrm{b}}^{(\mu, k)}$ is modified to

$$
P_{\mathrm{BER} \text { IBPA }}^{(\mu, k)}=\frac{2 \cdot\left(1-\frac{1}{\sqrt{M_{\mu}}}\right)}{\log _{2}\left(M_{\mu}\right)} \cdot \operatorname{erfc}\left(\sqrt{\frac{3 \cdot p_{\mathrm{IBPA}}^{(\mu, k)} \cdot \xi^{(\mu, k)} \cdot P_{\mathrm{S} \text { IBPA }}^{(\mu, k)}}{4 \cdot U_{\mathrm{R}}^{2} \cdot\left(M_{\mu}-1\right)}}\right)
$$


Afterwords, the PA algorithm is applied according to Figure 3-18b and Figure 318a. Firstly, the remaining non-allocated power is equally distributed over the MIMO active layers. Secondly, an equal-SNR PA technique for Non-Equal Power Distribution is introduced.

The drawback of the investigated IBPA solution which is the fixed data rate per time slot can be avoided for improving the overall performance. First four bits are equally allocated like the first four bits in the IBPA with fixed data rate method. Within this research, the 8-QAM is excluded since the transmit power per MIMO layer expression does not consider the transmit power of an 8-QAM accurate enough. The updated QAM constellation size is $(16,0,0,0)$. The QAM constellation with the minimum transmit power is selected among the different transmission modes achieved due to the bit allocation in different layers. After that, bits are individually allocated as long as the overall transmit power $P_{\mathrm{s} \text { IBPA }}^{(k)}$ is lower than one. Because of that, the QAM transmission mode becomes $(16,2,0,0)$ in the third row of Figure 3$19 \mathrm{~b},(32,2,0,0)$ in the fourth row, $(32,4,0,0)$ in the fifth, $(64,4,0,0)$ in the sixth and $(128,4,0,0)$ in the seventh one. In this moment, due to allocation, an extra bit means exceed the transmit power limit, the definitive QAM constellation results $(128,4,0,0)$.

Besides, according to Figure 3-19a and Figure 3-19b, PA techniques are applied. The remaining not allocated power is equally distributed over the MIMO active layers. And additionally, an equal-SNR Power Allocation (SNR-PA) technique for Non-Equal Power Distribution is introduced.

Simulations An optimized scheme would now use the specific transmission mode, guaranteeing the minimum BER for each symbol block $k$. As highlighted in Figure 322 and Figure 3-23 by using the best transmission mode per time slot over frequency non-selective MIMO channels with weak and strong correlation respectively, a further minimizing of the overall BER can be obtained. The drawback is the higher signalling overhead.

Due to the QAM transmission modes that are previously defined in the fixed 


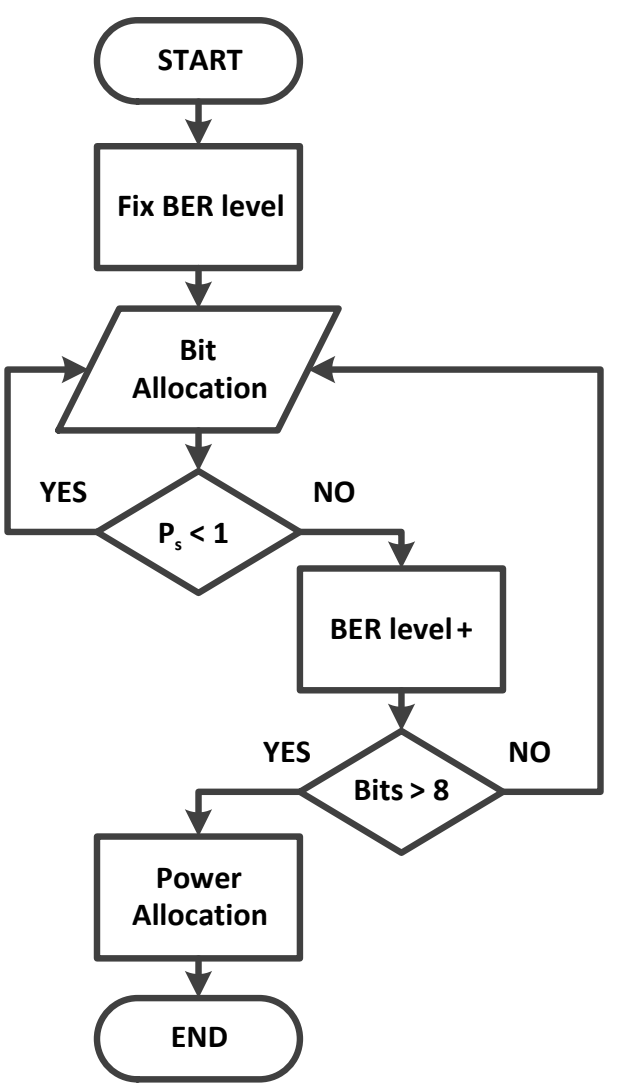

(a) Flowchart

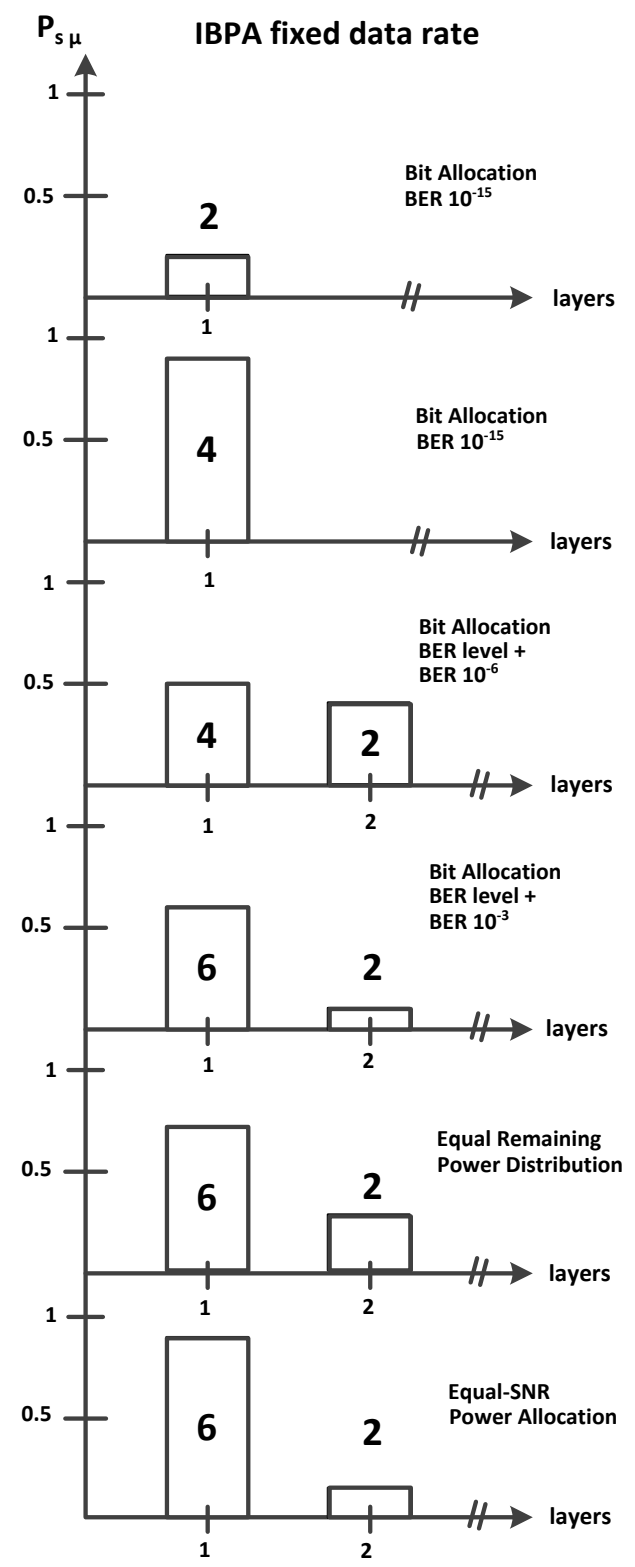

(b) Step-by-step IBPA algorithm example

Figure 3-18: Iterative Bit- and Power Allocation scheme at a fixed data rate 


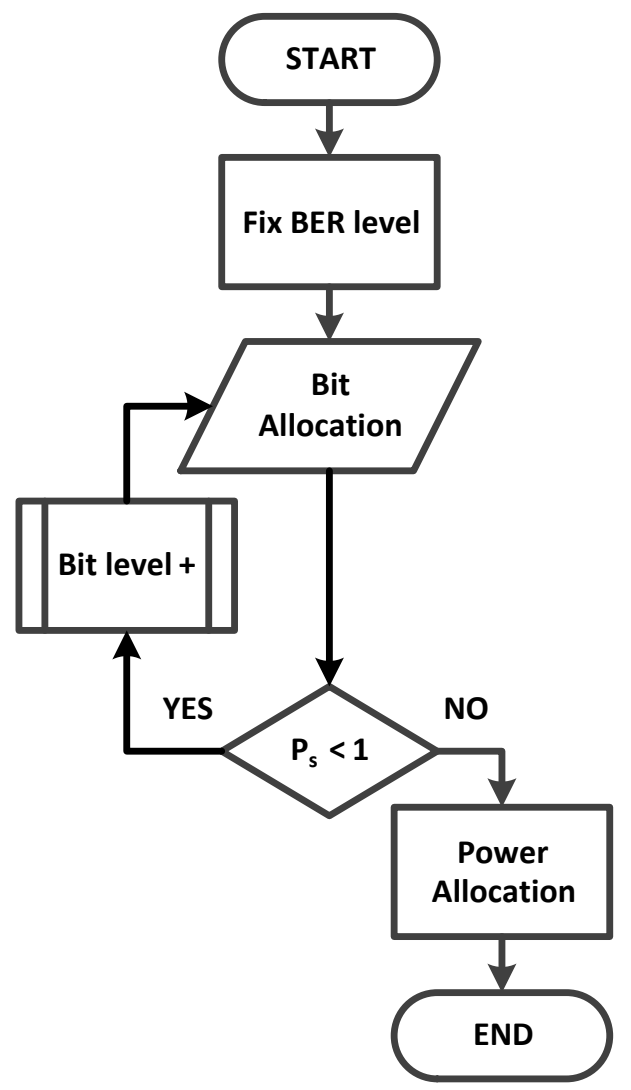

(a) Flowchart

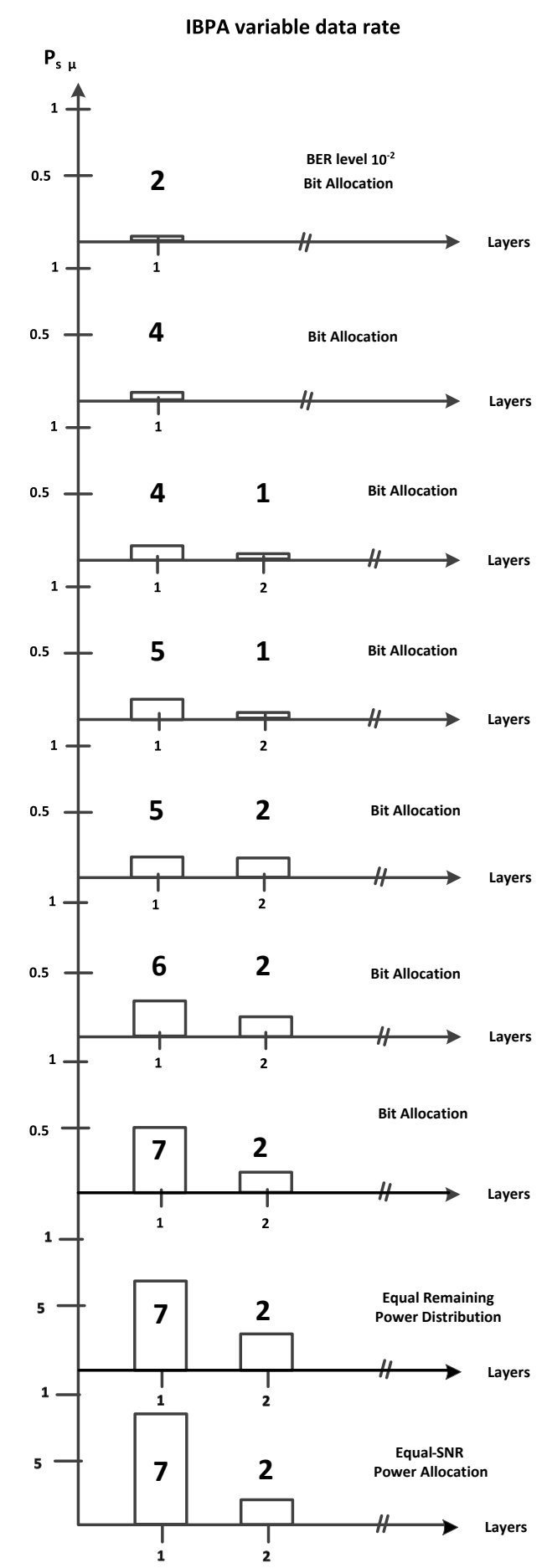

(b) Step-by-step IBPA algorithm example

Figure 3-19: Iterative Bit- and Power Allocation scheme at a variable data rate 
QAM constellation approach, some kind of rigidity in terms of resource allocation is introduced. In order to increase the degree of freedom in the MIMO performance design, iterative bit allocation with adaptive QAM constellation sizes per time slot is developed in this contribution. Furthermore, an optimal transmit power allocation with equal-SNR not uniformly distributed per active layers is used for minimizing the overall BER.

Table 3.4 allows comparing the effect of the investigated correlated channel profiles listed in Table 3.2 over BER performance in $(4 \times 4)$ MIMO system with IBPA fixed data rate. Due to the increasing correlation effect from (CP-1) to (CP-2), the bit-error probability increases.

The resultant BER curves are depicted in Figure 3-22 and Figure 3-23 when transmitting at $8 \mathrm{bit} / \mathrm{s} / \mathrm{Hz}$ using the iterative bit and power allocation algorithm previously described. Comparing the IBPA fixed data rate BER curves, in both investigated channels' profiles weak and strong correlation, show the significant BER performance improvement compared with the fixed QAM constellation sizes after applying optimal resources allocation techniques.

Table 3.4: Investigated channel profiles assuming a $(4 \times 4)$ MIMO system with IBPA fixed data rate at $E_{\mathrm{s}} / N_{0}=20 \mathrm{~dB}$

\begin{tabular}{|c|c|c|c|}
\hline Profile & $\mathrm{bit} / \mathrm{s} / \mathrm{Hz}$ & QAM Transmission modes & BER \\
\hline CP-1 & 8 & $(64,4,0,0)$ & $1.6 \cdot 10^{-4}$ \\
\hline $\mathrm{CP}-2$ & 8 & $(64,4,0,0)$ & $1.8 \cdot 10^{-4}$ \\
\hline
\end{tabular}

The constraint in the investigated IBPA algorithm concerning to the requirement of fixed data rate at a given bit/s/Hz per time slot can be modified for a variable data rate approach where the given bit/s/Hz in average is assured with the aim to improve the overall BER performance. From Table 3.5 it is possible to compare the effect of the investigated correlated channel profiles, listed on Table 3.2, over BER performance in $(4 \times 4)$ MIMO system with IBPA variable data rate. Depending on the transmitting number of bits the QAM transmission mode is modified. The higher 
the transmit data rate, the higher the BER performance.

Figure 3-24 shows the comparison between MIMO system performances when using fixed and variable data rate and transmitting an average data throughput of $8 \mathrm{bit} / \mathrm{s} / \mathrm{Hz}$ over frequency non-selective $(4 \times 4)$ MIMO channels with and without antenna correlation. As highlighted by the BER curves, from the variable data rate schema a noticeable better BER performance is obtained.

Table 3.5: Investigated channel profiles assuming a $(4 \times 4)$ MIMO system with IBPA variable data rate at $E_{\mathrm{s}} / N_{0}=20 \mathrm{~dB}$

\begin{tabular}{|c|c|c|c|}
\hline Profile & $\mathrm{bit} / \mathrm{s} / \mathrm{Hz}$ & QAM Transmission modes & BER \\
\hline CP-1 & 7 & $(32,4,0,0)$ & $1.4 \cdot 10^{-6}$ \\
\hline $\mathrm{CP}-1$ & 8 & $(32,4,2,0)$ & $3.2 \cdot 10^{-5}$ \\
\hline $\mathrm{CP}-1$ & 12 & $(64,16,4,0)$ & $7.2 \cdot 10^{-3}$ \\
\hline $\mathrm{CP}-2$ & 7 & $(32,4,0,0)$ & $5.8 \cdot 10^{-6}$ \\
\hline CP-2 & 8 & $(64,4,0,0)$ & $1.8 \cdot 10^{-4}$ \\
\hline CP-2 & 9 & $(128,4,0,0)$ & $2.5 \cdot 10^{-3}$ \\
\hline
\end{tabular}




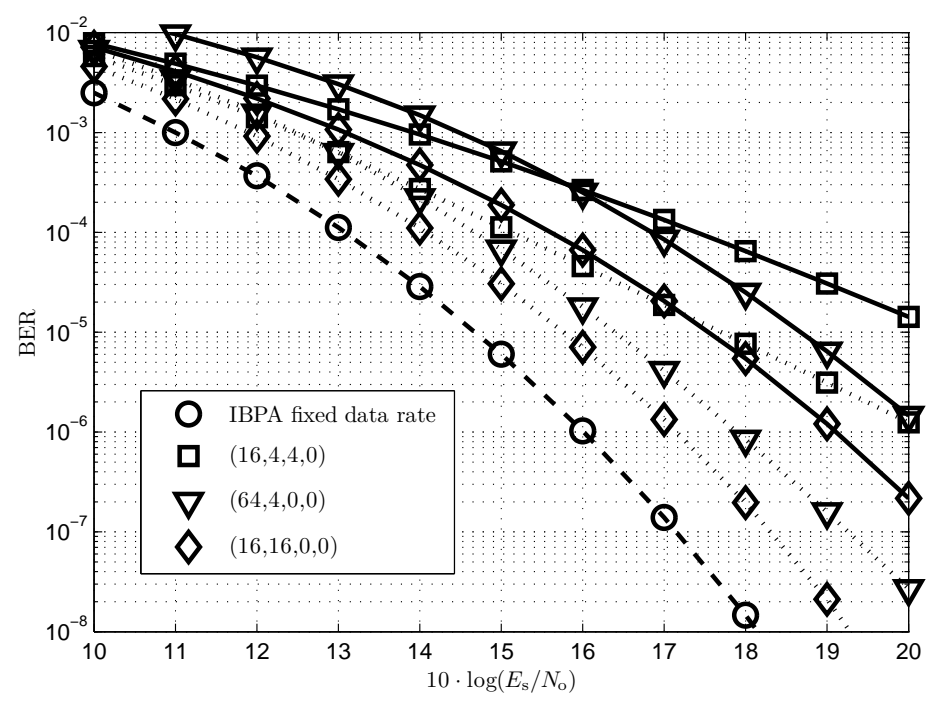

Figure 3-20: BER with IBPA fixed data rate algorithm (dashed line) compared to BER curves with equal-SNR PA (dotted line) and without PA (solid line) when using the transmission modes introduced in Table 3.1 and transmitting $8 \mathrm{bit} / \mathrm{s} / \mathrm{Hz}$ over frequency non-selective $(4 \times 4)$ MIMO channels with weak antenna correlation

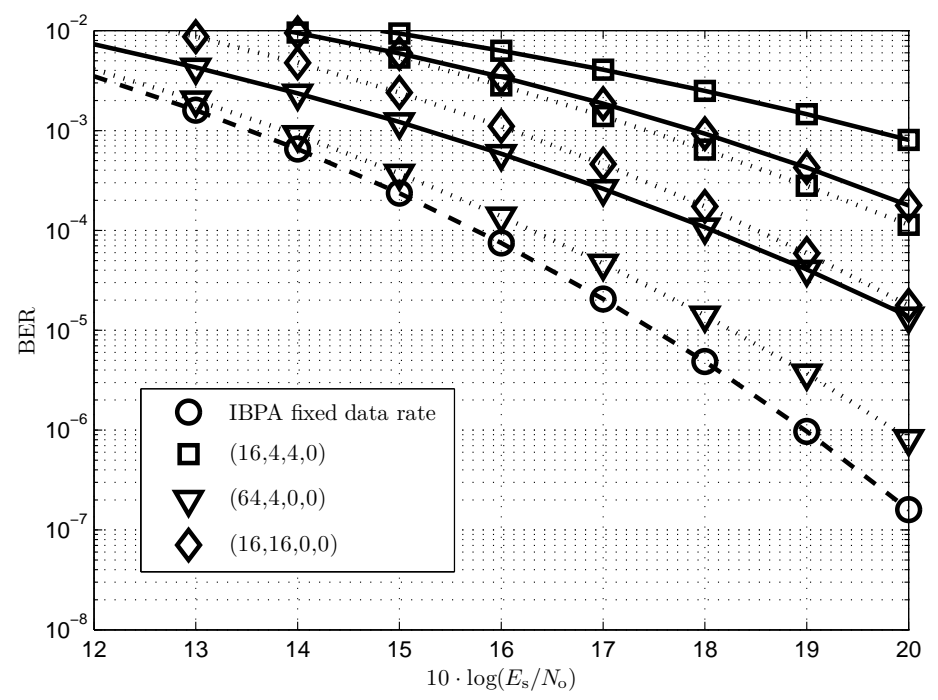

Figure 3-21: BER with IBPA fixed data rate algorithm (dashed line) compared to BER curves with equal-SNR PA (dotted line) and without PA (solid line) when using the transmission modes introduced in Table 3.3 and transmitting 8 bit $/ \mathrm{s} / \mathrm{Hz}$ over frequency non-selective $(4 \times 4)$ MIMO channels with strong antenna correlation 


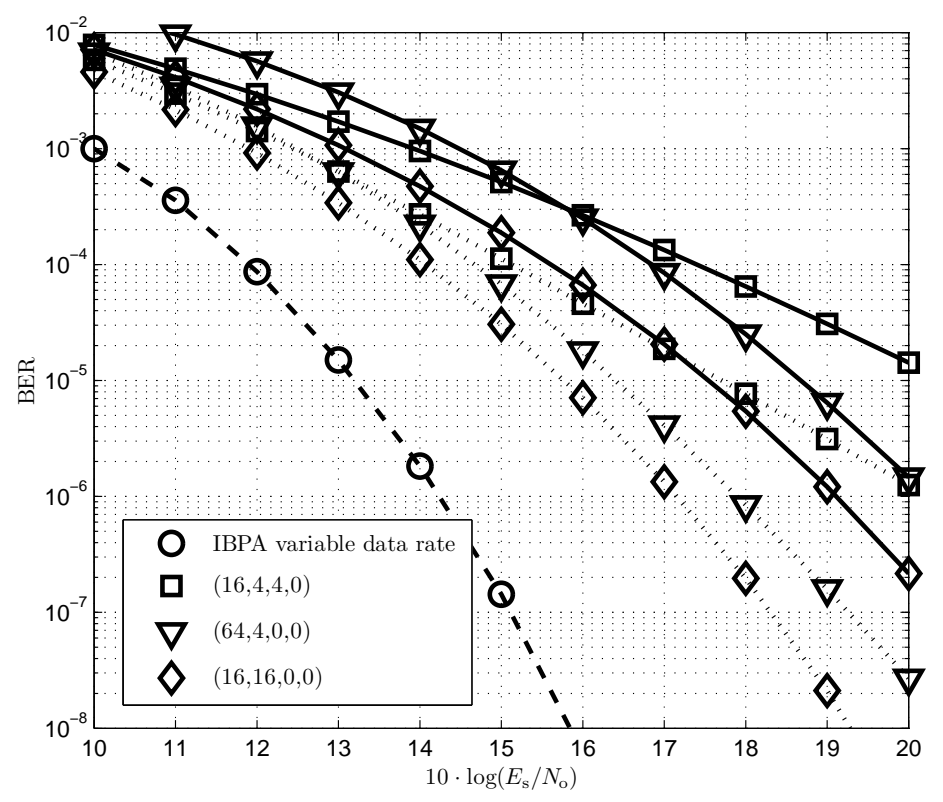

Figure 3-22: BER with IBPA variable data rate algorithm (dashed line) compared to BER curves with equal-SNR PA (dotted line) and without PA (solid line) when using the transmission modes introduced in Table 3.1 and transmitting $8 \mathrm{bit} / \mathrm{s} / \mathrm{Hz}$ over frequency non-selective $(4 \times 4)$ MIMO channels with weak antenna correlation

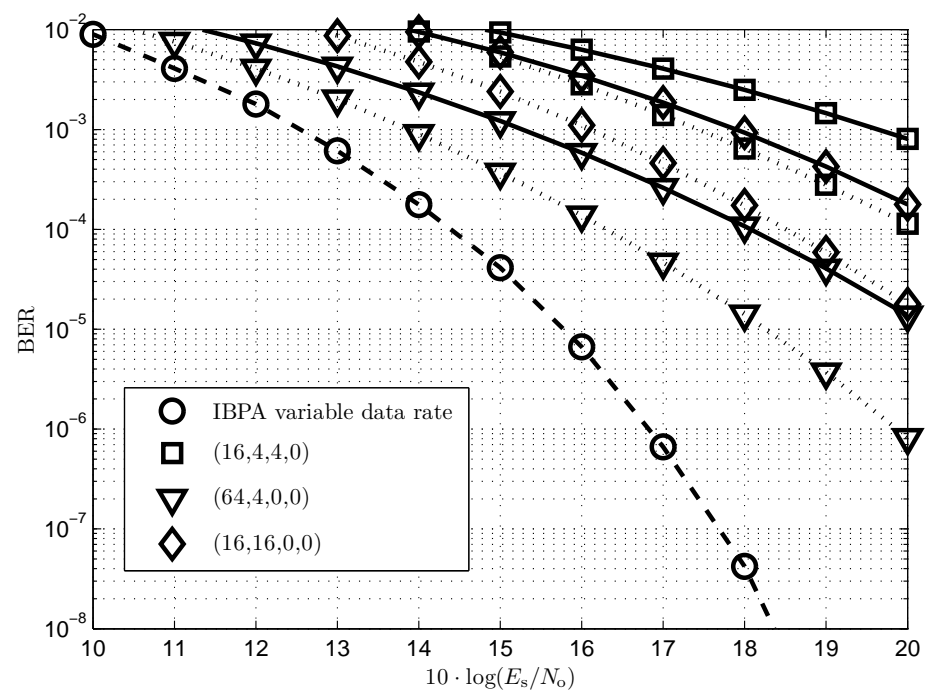

Figure 3-23: BER with IBPA variable data rate algorithm (dashed line) compared to BER curves with equal-SNR PA (dotted line) and without PA (solid line) when using the transmission modes introduced in Table 3.3 and transmitting $8 \mathrm{bit} / \mathrm{s} / \mathrm{Hz}$ over frequency non-selective $(4 \times 4)$ MIMO channels with strong antenna correlation 


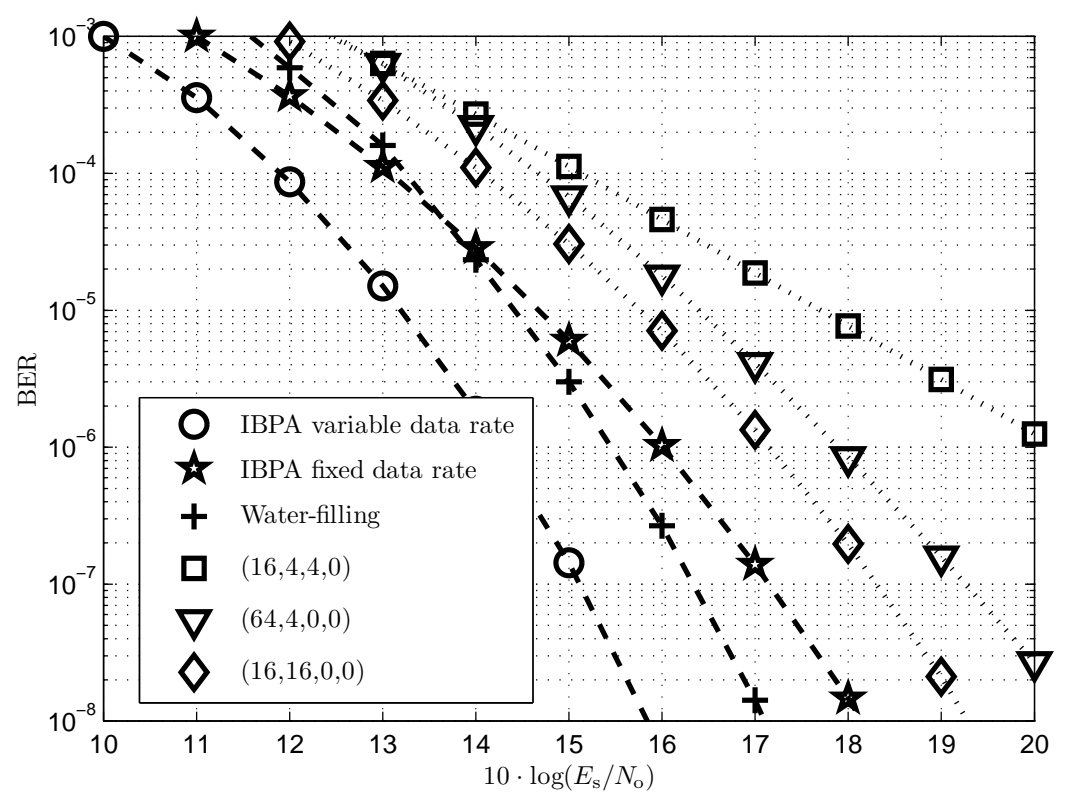

Figure 3-24: Comparison PA techniques developed. IBPA at a fixed data rate, IBPA at a variable data rate, Water-filling and Equal-SNR PA when transmitting an average data throughput of $8 \mathrm{bit} / \mathrm{s} / \mathrm{Hz}$ over frequency non-selective $(4 \times 4)$ weak correlated MIMO channels 


\subsection{Geometric Mean Decomposition}

Matrix decompositions play an important role in analysing the capacity and designing the transceiver for MIMO channels [29]. This geometric mean decomposition (GMD) is applied to signal processing and to the design of telecommunication networks. This decomposition is used while trying to optimize the performance of MIMO systems. The GMD can be used to create identical SNR for each decoupled subchannel.

GMD is based on a theorem from linear algebra which says that given a $(\mu \times \nu)$ complex matrix A, it considers the unitary decomposition [25].

$$
\mathbf{A}=\mathbf{Q} \cdot \Sigma \cdot \mathbf{P}^{\mathrm{H}}
$$

where $\mathbf{Q}$ and $\mathbf{P}$ have orthogonal columns. Thus, $\boldsymbol{\Sigma}$ is a real upper triangular matrix with all diagonal elements equal to the geometric mean of the positive singular values of $\mathbf{A}$

$$
r_{i i}=\left(\prod_{i=1}^{L_{\mathrm{a}}} \sqrt{\xi^{(i)}}\right)^{\frac{1}{L_{\mathrm{a}}}}
$$

where $\sqrt{\xi^{(i)}}>0$ and $1 \leq L_{\mathrm{a}} \leq \min \left(n_{\mathrm{R}}, n_{\mathrm{T}}\right)$. Thus, the $\sqrt{\xi^{(i)}}\left(\right.$ for $\left.i=1, \ldots, L_{\mathrm{a}}\right)$ are the singular values of $\mathbf{H}, L_{\mathrm{a}}$ the number of active layers and $r_{i i}$ the geometric mean of the positive singular values.

MIMO systems have been the subject of intensive research over the past years due to the fact that it supports rather higher data rates and reliability than SISO systems [24]. In this doctoral thesis, the MIMO system has been modelled as follows:

$$
\mathbf{u}_{\mathrm{e}}=\mathbf{H} \cdot \mathbf{u}_{\mathrm{s}}+\mathbf{n}
$$

where $\mathbf{u}_{\mathrm{e}}$ corresponds to the $\left(n_{\mathrm{R}} \times 1\right)$ received vector, $\mathbf{H}$ is the $\left(n_{\mathrm{R}} \times n_{\mathrm{T}}\right)$ channel matrix, $\mathbf{u}_{\mathrm{s}}$ is the $\left(n_{\mathrm{T}} \times 1\right)$ transmitted vector and $\mathbf{n}$ is the AWGN vector. Furthermore, it is assumed that the coefficients of the $\left(n_{\mathrm{R}} \times n_{\mathrm{T}}\right)$ channel matrix $\mathbf{H}$ are independently Rayleigh distributed with equal variance, and that the number of transmit antennas 
$n_{\mathrm{T}}$ equals to the number of receive antennas $n_{\mathrm{R}}$.

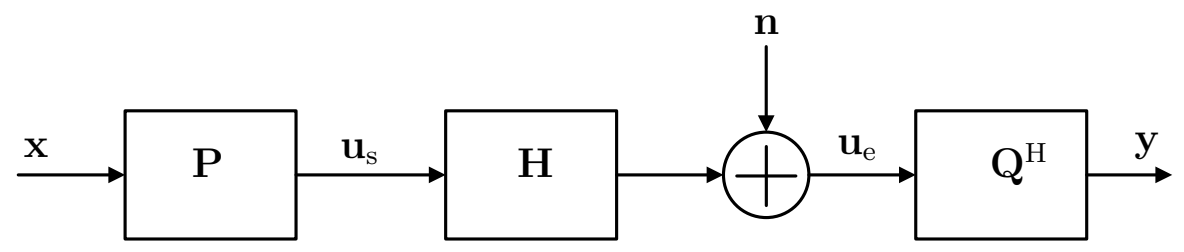

Figure 3-25: Pre- and post processing GMD model for MIMO systems

By using the GMD technique, pre- and post signal processing is required. According to Figure 3-25 and due to the MIMO channel decomposed as $\mathbf{H}=\mathbf{Q} \cdot \mathbf{\Sigma} \cdot \mathbf{P}^{\mathrm{H}}$, the following equivalent system is obtained (Figure 3-26)

$$
\mathbf{y}=\mathbf{Q}^{\mathrm{H}} \cdot \mathbf{u}_{\mathrm{e}}=\mathbf{Q}^{\mathrm{H}}(\mathbf{H} \cdot \mathbf{P} \cdot \mathbf{x}+\mathbf{n})=\mathbf{\Sigma} \cdot \mathbf{x}+\mathbf{w}
$$

where $\mathbf{H} \cdot \mathbf{P} \cdot \mathbf{u}_{\mathbf{s}}$ is the pre-processed transmit data vector and $\mathbf{Q}^{\mathrm{H}} \cdot \mathbf{u}_{\mathrm{e}}$ is the post-processed data vector at the receiver side. The noise is also affected by the post-processing process as $\mathbf{w}=\mathbf{Q}^{\mathrm{H}} \cdot \mathbf{n}$.

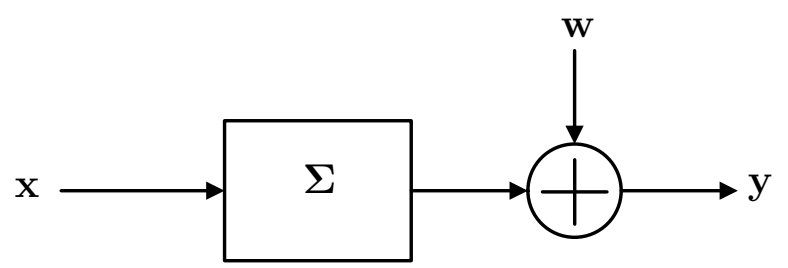

Figure 3-26: Simplified GMD model for MIMO systems after signal processing

In order to understand the effect produced by this GMD technique easily, a $(4 \times 4)$ uncorrelated MIMO channel is analysed. Taking into consideration different numbers of active layers, the real upper triangular matrix $\boldsymbol{\Sigma}$ becomes

$$
\mathbf{H}^{(4 \times 4)}=\left(\begin{array}{cccc}
1,394 & 0,729 & 0,189 & 1,029 \\
0,789 & -1,649 & 0,240 & -0,783 \\
-0,545 & -1,610 & 0,307 & 2,018 \\
1,116 & 0,319 & -1,196 & -0,340
\end{array}\right),
$$




$$
\begin{aligned}
& \boldsymbol{\Sigma}^{\left(L_{\mathrm{a}}=1\right)}=\left(\begin{array}{cccc}
2,845 & 0 & 0 & 0 \\
0 & 0 & 0 & 0 \\
0 & 0 & 0 & 0 \\
0 & 0 & 0 & 0
\end{array}\right), \boldsymbol{\Sigma}^{\left(L_{\mathrm{a}}=2\right)}=\left(\begin{array}{cccc}
2,474 & -0,695 & 0 & 0 \\
0 & 2,474 & 0 & 0 \\
0 & 0 & 0 & 0 \\
0 & 0 & 0 & 0
\end{array}\right) \\
& \boldsymbol{\Sigma}^{\left(L_{\mathrm{a}}=3\right)}=\left(\begin{array}{cccc}
2,283 & -0,609 & 0,647 & 0 \\
0 & 2,283 & -0,273 & 0 \\
0 & 0 & 2,283 & 0 \\
0 & 0 & 0 & 0
\end{array}\right), \boldsymbol{\Sigma}^{\left(L_{\mathrm{a}}=4\right)}=\left(\begin{array}{cccc}
1,865 & -1,294 & 0,903 & -0,866 \\
0 & 1,865 & 0,428 & -0,411 \\
0 & 0 & 1,865 & 0,155 \\
0 & 0 & 0 & 1,865
\end{array}\right)
\end{aligned}
$$

Furthermore, Figure 3-27 highlights the probability of having singular values higher than a certain value by analysing the CCDF curves. The GMD technique yields a solution to maximizing problems due to the smallest diagonal element of $\boldsymbol{\Sigma}$ is as large as possible. In MIMO systems, this minimizes the worst possible error in the transmission process. Other applications of the GMD are to precoders for suppressing inter-symbol interferences.

Simulations In order to describe the receiver-side antennas correlation effect on the channel performance a $(4 \times 4)$ frequency non-selective GMD-aided MIMO system with and without correlation effect is simulated. In Figure 3-28 the PDF of the comparison between a three-active-layers-channel with and without correlation is shown. Furthermore, the CCDF curves are depicted in Figure 3-29.

After having analysed the uncorrelated GMD-aided MIMO channel profile, the resulting BER curves with and without equal-SNR PA are highlighted in Figure 330 for uncorrelated channel where perfect interference cancellation is assumed. In Figure 3-31 the BER curves under high correlation rate are shown. It is noted from BER curves that PA technique can be helpful in combination with an appropriate transmission modes to minimize the overall BER performance. 


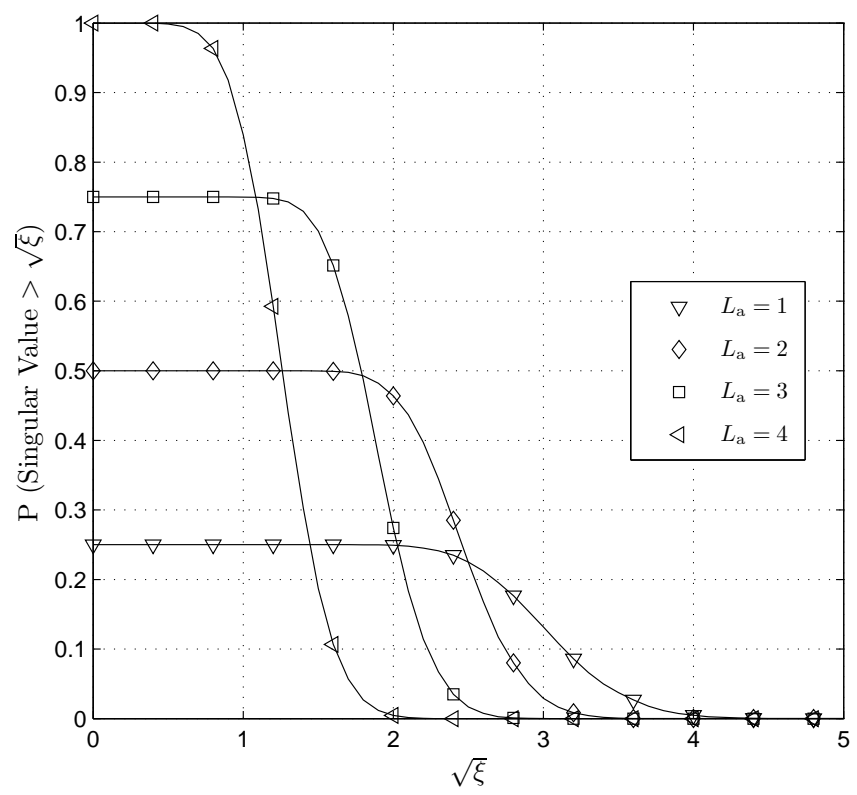

Figure 3-27: CCDF of the layer-specific distribution for uncorrelated frequency nonselective $(4 \times 4)$ GMD-aided MIMO channel

\subsection{Tomlinson-Harashima Precoding}

In this section, a well-known non-linear pre-equalization technique,namely TomlinsonHarashima precoding (THP), is proposed for transmission over a frequency nonselective $(4 \times 4)$ GMD-aided MIMO communication system.

In Figure 3-32, a system model in order to cancellate the successive inter-symbol interference is presented. The block diagram shows a decision-feedback equalization in the receive side which consists of a decision modulo, a symbol delay and a feedback channel matrix $\mathbf{H}_{\mathrm{t}}$ defined by

$$
\mathbf{H}_{\mathrm{t}}=\boldsymbol{\Sigma}-\operatorname{diag}(\boldsymbol{\Sigma}) \cdot \mathbf{I}
$$

Via the feedback channel matrix, the interference caused by already detected symbols is cancelled. Since $\mathbf{H}_{\mathrm{t}}$ is strictly an upper triangular, a causal loop for successive detection and interference cancellation outcome [26]. The main drawback that this successive interference cancellation technique presents is the increasing power of the precoded signals. 


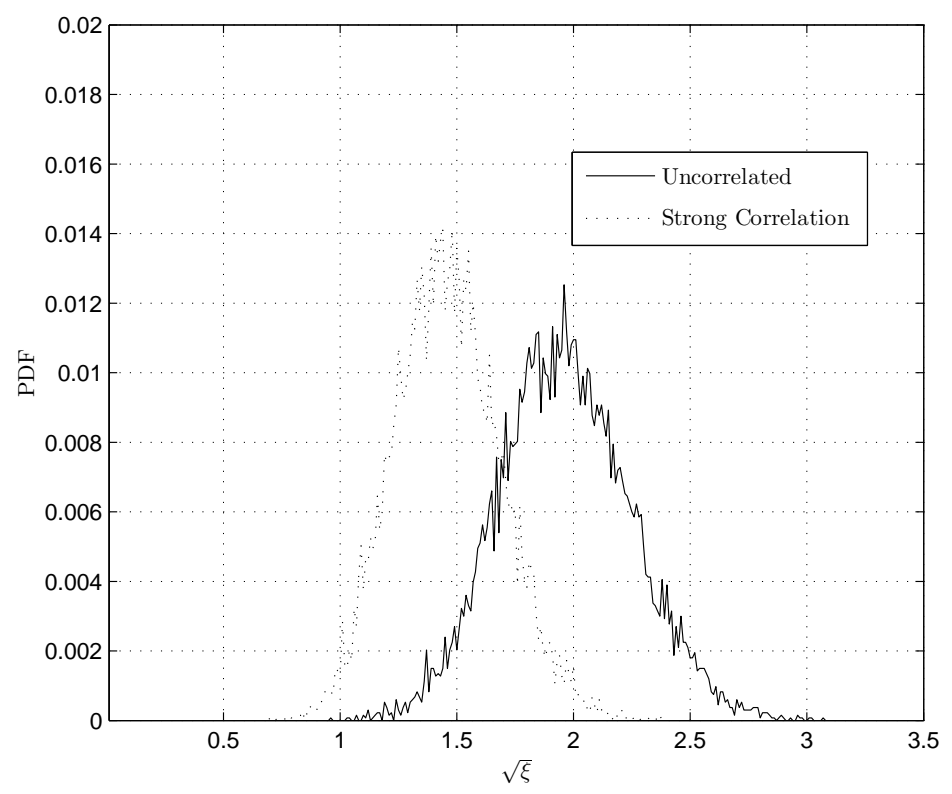

Figure 3-28: PDF of the comparison between the effect of the uncorrelation and the strong correlation in a frequency non-selective $(4 \times 4)$ GMD-aided MIMO system with $L_{\mathrm{a}}=3$

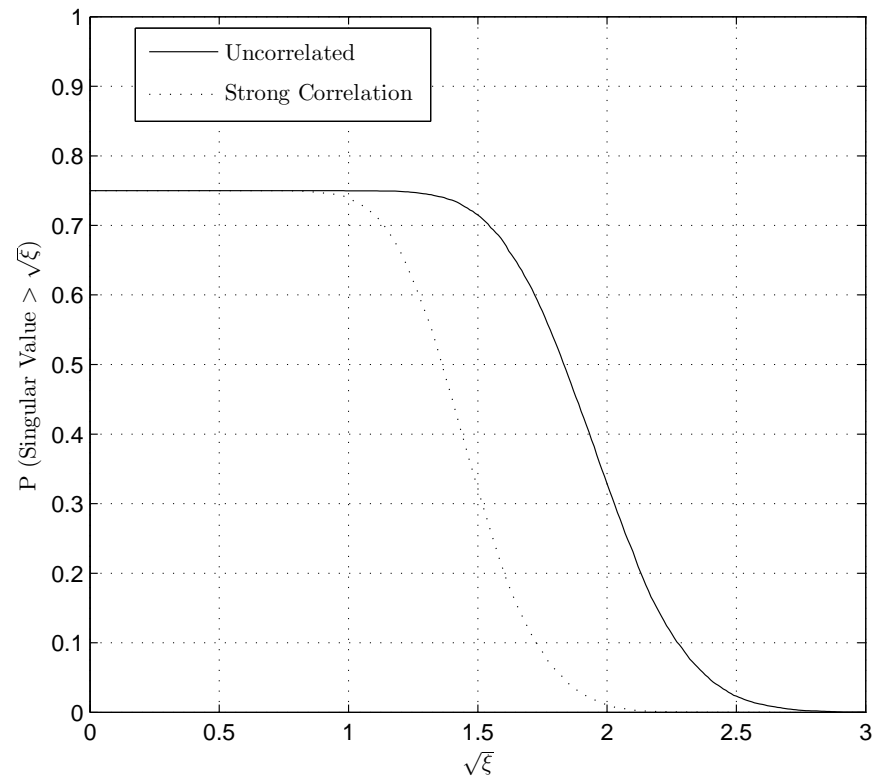

Figure 3-29: CCDF of the comparison between the effect of the uncorrelation and the strong correlation in a frequency non-selective $(4 \times 4)$ GMD-aided MIMO system with $L_{\mathrm{a}}=3$ 


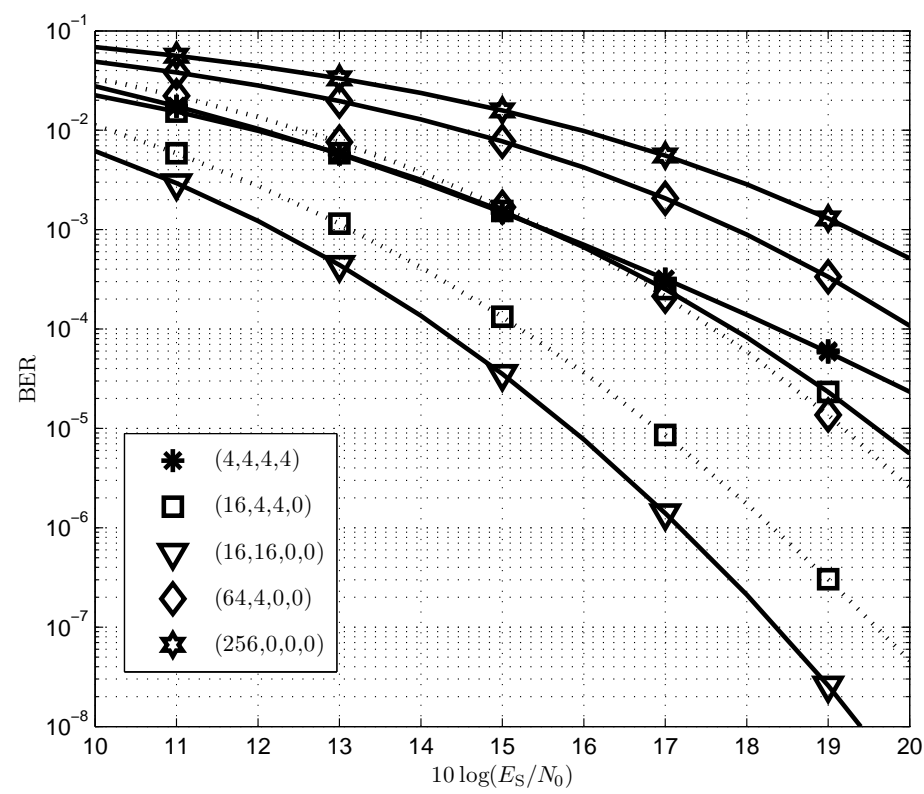

Figure 3-30: BER comparison of the uncorrelated frequency non-selective $(4 \times 4)$ GMD-aided MIMO system with equal-SNR PA technique (dotted lines) and without (solid lines) assuming perfect interference cancellation

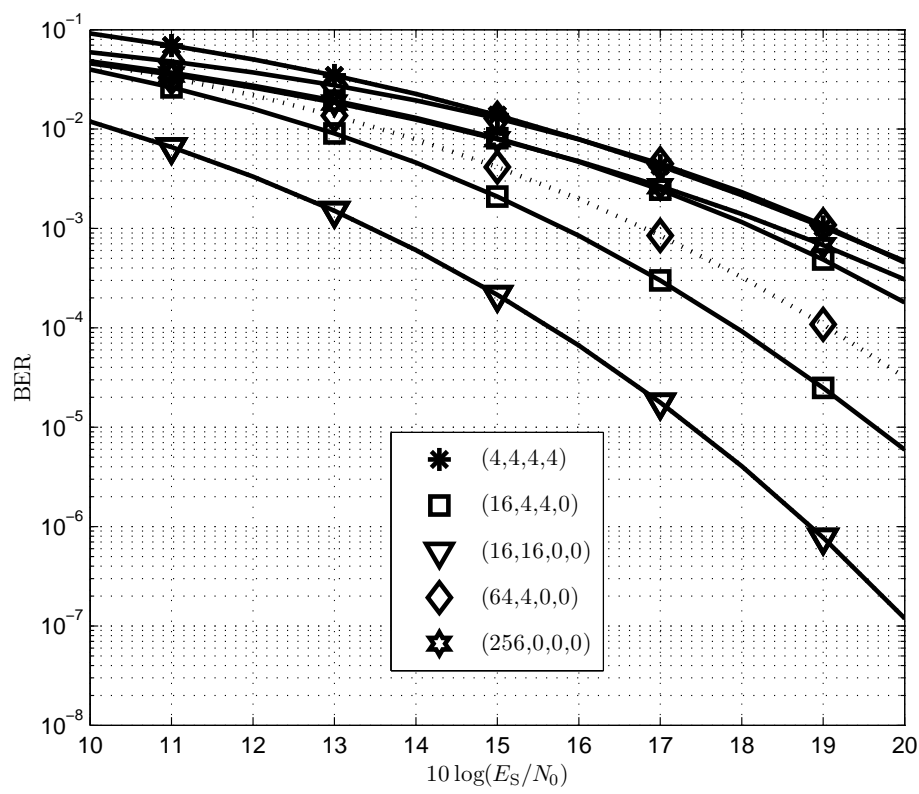

Figure 3-31: BER comparison of the correlated frequency non-selective $(4 \times 4)$ GMDaided MIMO system with equal-SNR PA technique (dotted lines) and without (solid lines) assuming perfect interference cancellation 


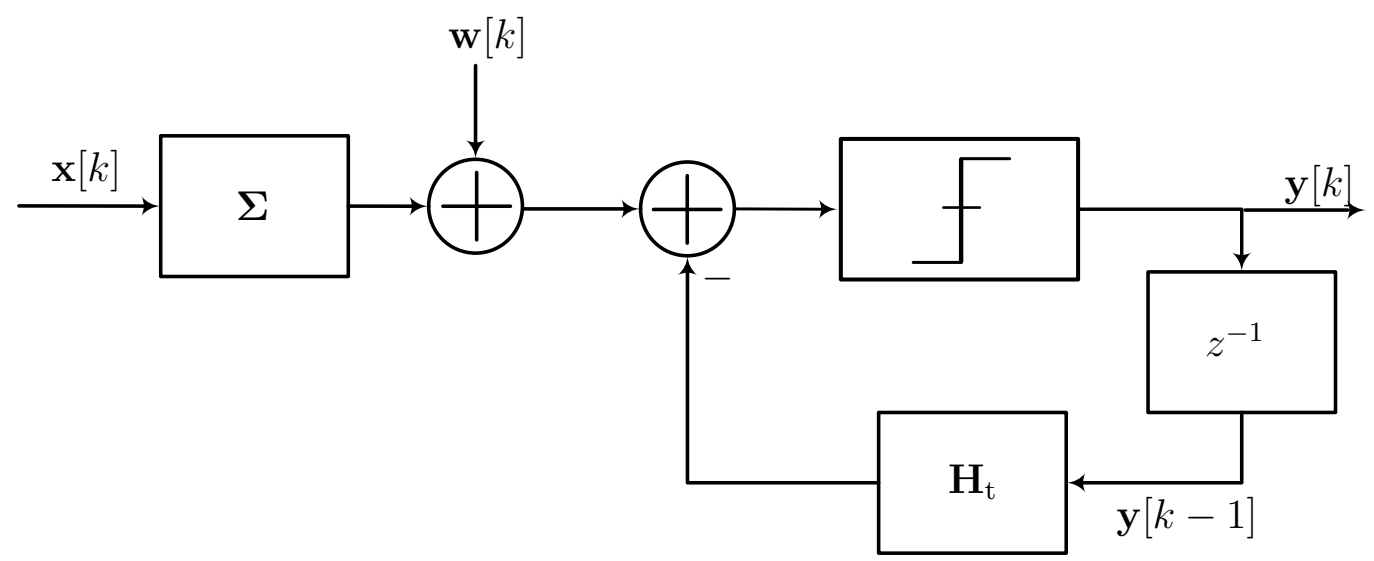

Figure 3-32: Successive Interference Cancellation model for MIMO systems in the receiver side

Therefore, in this way, THP technique can be interpreted as moving the feedback part of successive interference cancellation technique to the transmitter side. Thereby, two deficiencies of the previous system scheme can be overcome: since the operation is performed at the transmitter where signals are perfectly known, no error propagation can occur and for equalization, no immediate decisions are required [38].

In Figure 3-33, the THP system model over a MIMO channel is depicted where $\mathbf{x}[k]$ corresponds to the $\left(n_{\mathrm{R}} \times 1\right)$ transmitted vector followed by a modulo reduction (MOD) to suppress the power enhancement, a symbol delay and a feedback channel matrix $\mathbf{H}_{\mathrm{t}}$ with an upper-triangular structure to obtain the transmit signal $\mathbf{s}[k]$ after the THP process. Furthermore, $\boldsymbol{\Sigma}$ is the real upper triangular matrix with all diagonal elements equal to the geometric mean of the positive singular values of the $\left(n_{\mathrm{R}} \times n_{\mathrm{T}}\right)$ channel matrix (due to the required pre- and post GMD processing techniques), $\mathbf{y}[k]$ is the $\left(n_{\mathrm{T}} \times 1\right)$ received vector and $\mathbf{w}[k]$ is the AWGN GMD post-processed vector. Assuming that perfect channel state information is available, $\mathbf{H}_{\mathrm{t}}$ is perfectly characterized at the transmitter side.

Given the transmit data symbols $\mathbf{x}[k]$, the channel transmit symbols are recursively calculated as

$$
\mathbf{s}[k]=\operatorname{MOD}\left\{\mathbf{x}[k]-\mathbf{H}_{\mathbf{t}} \cdot \mathbf{s}[k-1]\right\}
$$




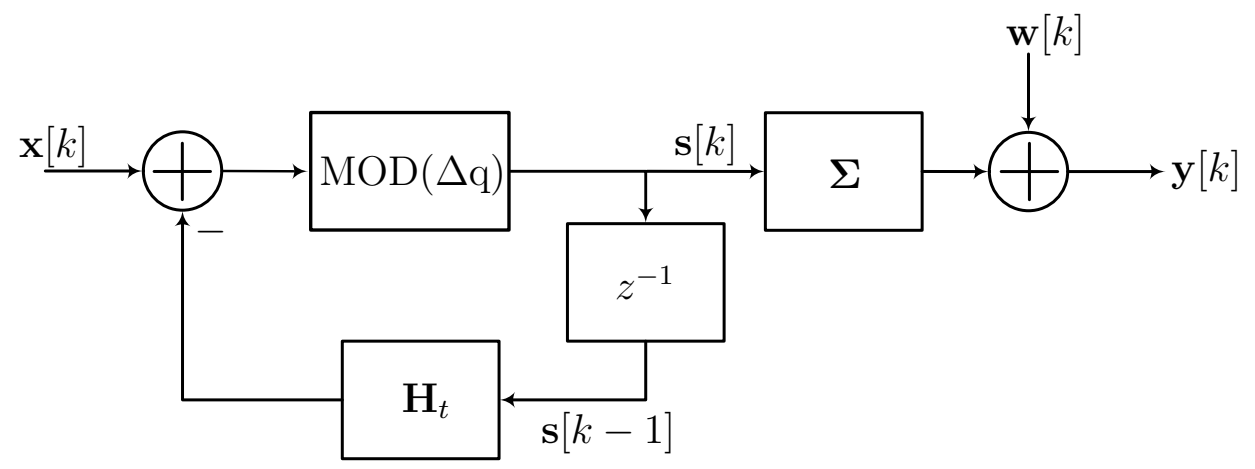

Figure 3-33: Tomlinson-Harashima precoding model for MIMO systems

Since successive interference cancellation strategy would increase transmit power, THP modulo reduces the real and imaginary part of the transmit symbols into the boundary constellation of width of the modulo operator [17]. This operator is defined by

$$
\operatorname{MOD}(\Delta \cdot q)=\operatorname{MOD}\left(2 \cdot U_{\mathrm{s}} \cdot q\right)
$$

where $\Delta$ is the distance between two adjacent symbols, $U_{\mathrm{s}}$ denotes the half-level transmit amplitude and $q=\sqrt{M}$, being $M$ the index modulation in every active MIMO layer.

Simulations In order to describe the THP technique effect on the channel performance a $(4 \times 4)$ frequency non-selective GMD-aided MIMO system is simulated.

Figure 3-34 shows a comparison between the Perfect Interference Cancellation technique and the Successive Interference Cancellation in the receiver side (Figure 332) for a $(4,4,4,4)$ QAM transmission mode. Hence, it turns out the existence of error propagation due to the activation of all MIMO layers. Consequently, also in GMDaided MIMO systems not all MIMO layers have to be activated in order to achieve the best BER performance. Furthermore, Figure 3-35 shows the perfect interference cancellation made by the THP technique for a $(16,16,0,0)$ QAM transmission mode due to the error propagation is eliminated.

From Figure 3-36 and Figure 3-37 the correlation effect is analysed by using the scatter plot tool. Figure 3-36a and Figure 3-37a display the Gray-coded constellation 


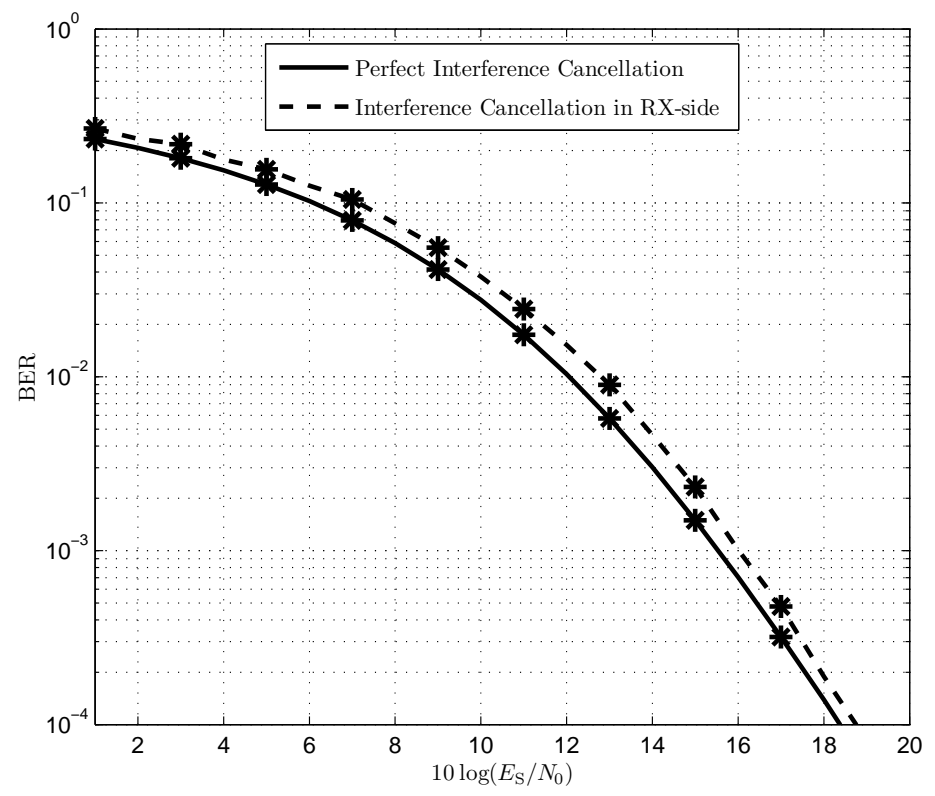

Figure 3-34: BER comparison between the Perfect Interference Cancellation technique and the Successive Interference Cancellation in the receiver side for $(4,4,4,4)$ uncorrelated frequency non-selective $(4 \times 4)$ GMD-aided MIMO channel

before the THP modulo, and the Figure 3-36b and Figure 3-37b after this modulo. From these figures, the increasing dispersion in the constellation diagram due to the increasing correlation effect is highlighted. 


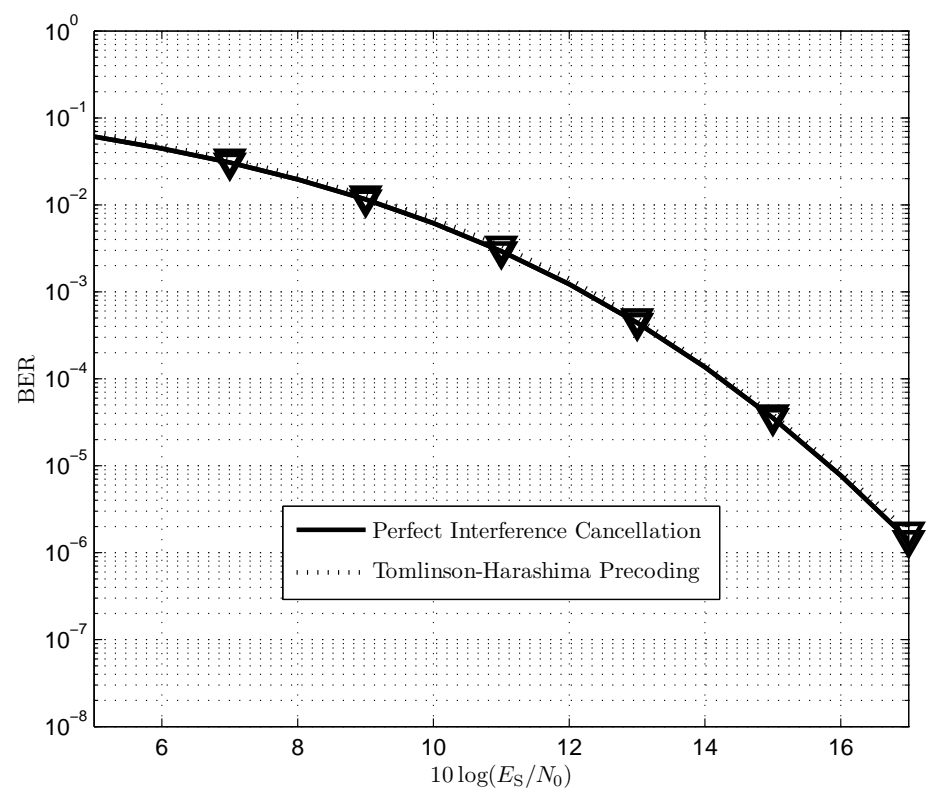

Figure 3-35: BER comparison between the Perfect Interference Cancellation technique and THP for $(16,16,0,0)$ uncorrelated frequency non-selective $(4 \times 4)$ GMDaided MIMO channel

\subsection{Comparison SVD-GMD}

In this section, the performance obtained by the Singular Value Decomposition technique and the one obtained by the Geometric Mean Decomposition is compared.

Firstly, the study of CCDF curves from over frequency non-selective $(4 \times 4)$ MIMO channels by using SVD and $L_{\mathrm{a}}=3$ active GMD layers is reported.

Furthermore, BER curves comparison between GMD (assuming perfect interference cancellation) and SVD techniques over frequency non-selective $(4 \times 4)$ uncorrelated MIMO channels is depicted in Figure 3-39 and over correlated MIMO channels in Figure 3-40. In both cases $8 \mathrm{bit} / \mathrm{s} / \mathrm{Hz}$ are transmitted by using the following transmission modes $(16,16,0,0)$ and $(64,4,4,0)$ with equal-SNR PA technique.

Following the investigation described in the power allocation section, different bit and power allocation strategies seem to be the most efficient solutions when minimizing the overall BER. In order to find out the best bit allocation performance per time slot, it is required to find the most appropriate QAM constellation with the minimum transmit power. Figure 3-41 and Figure 3-42 illustrate the previous BER with 


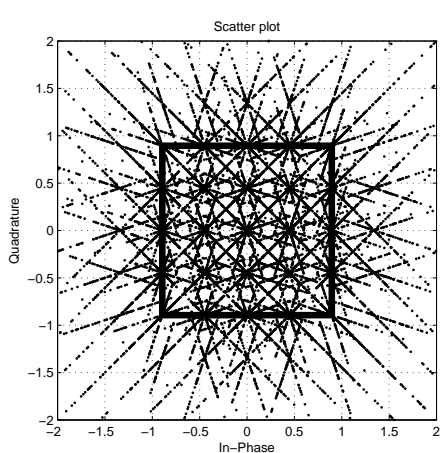

(a) Constellation diagram before Tomlinson-Harashima precoding modulo

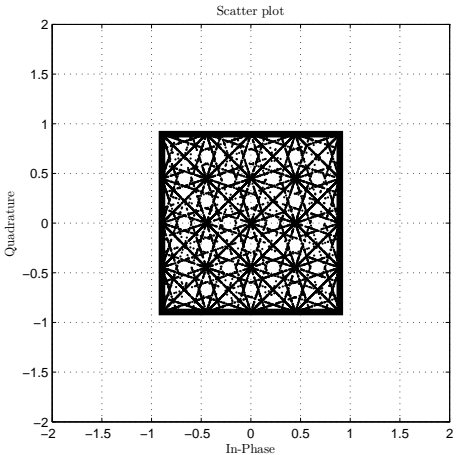

(b) Constellation diagram after Tomlinson-Harashima precoding modulo

Figure 3-36: Constellation diagram from transmit symbols with GMD technique and Tomlinson-Harashima precoding in the transmission side when the $(16,16,0,0)$ mode is transmitted over frequency non-selective $(4 \times 4)$ MIMO channels

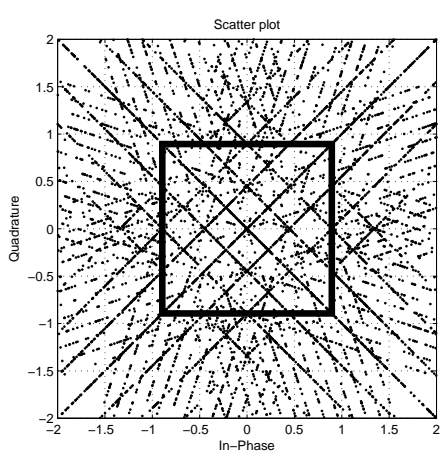

(a) Constellation diagram before Tomlinson-Harashima precoding modulo

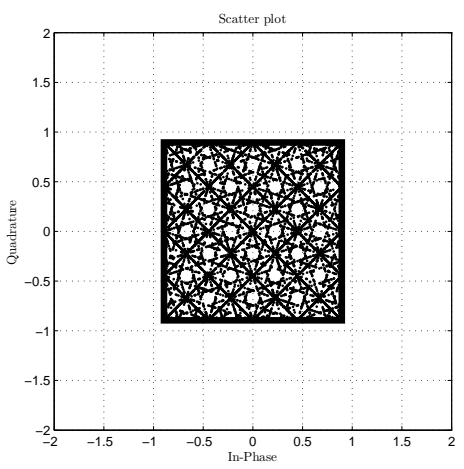

(b) Constellation diagram after Tomlinson-Harashima precoding modulo

Figure 3-37: Constellation diagram from transmit symbols with GMD technique and Tomlinson-Harashima precoding in the transmission side when the $(16,16,0,0)$ mode is transmitted over frequency non-selective $(4 \times 4)$ MIMO channels with correlation 


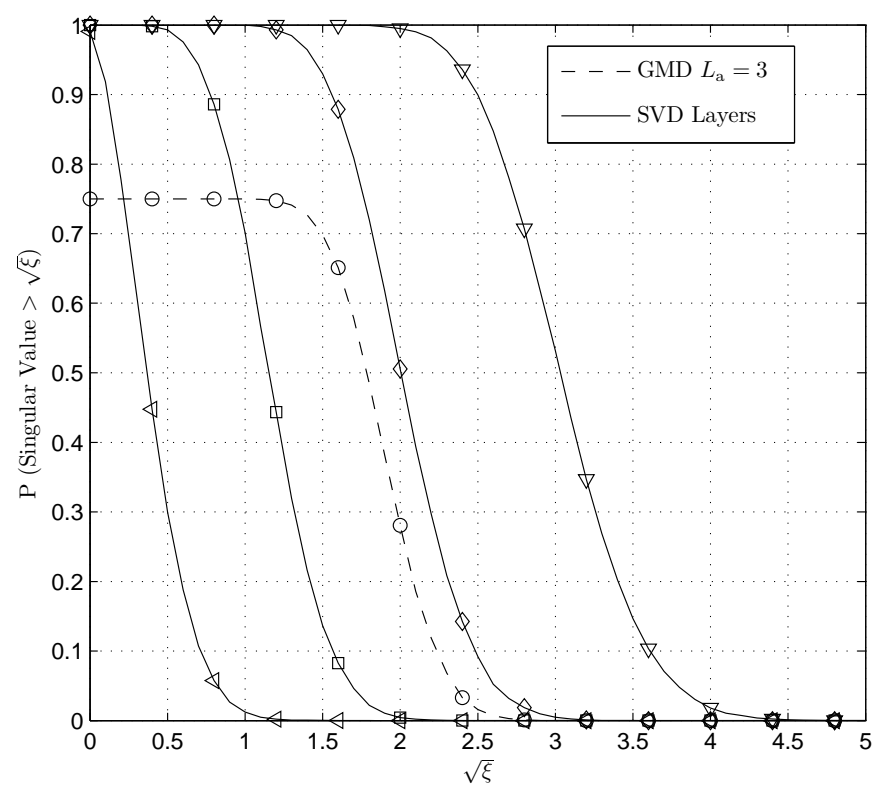

Figure 3-38: CCDF of the 3-active-layer-specific distribution for uncorrelated frequency non-selective $(4 \times 4)$ GMD-aided MIMO channel compared to the SVD-aided MIMO channel

equal-SNR PA techniques plus the method based on finding out the best transmission mode per time slot with and without correlation. 


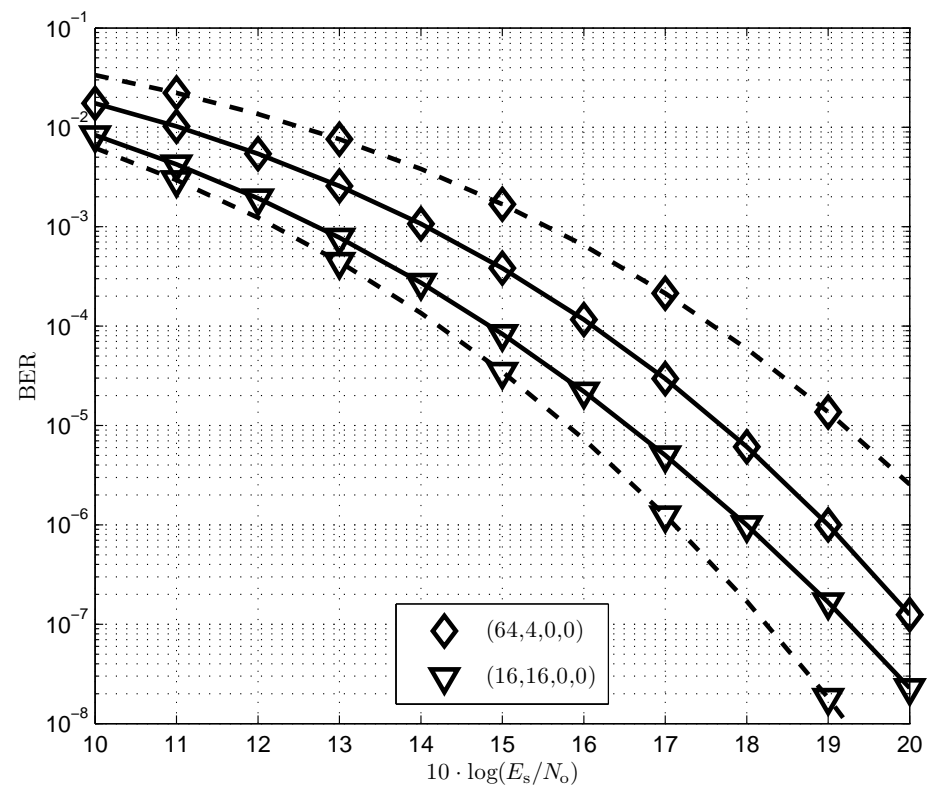

Figure 3-39: BER curves with GMD technique (dashed line) assuming perfect interference cancellation compared to BER curves with SVD (solid line) when using the transmission modes introduced in the legend with equal-SNR PA and transmitting $8 \mathrm{bit} / \mathrm{s} / \mathrm{Hz}$ over frequency non-selective $(4 \times 4)$ MIMO channels without antenna correlation

\subsection{Chapter Conclusions}

In this chapter of the doctoral thesis, the singular values from the different channel scenarios have been described by using a comparison between the lower and the higher singular value with the probability density function. The obtained simulation results allows to concluding that the antennas correlation effect increases the probability of having predominant layers. Consequently, the existence of predominant layers means the existence of weak layers.

One topic analysed through this chapter is the BER curves from different QAM transmission modes where is possible to observe clearly the importance of choosing an appropriate number of active layers with accurate QAM constellation size in order to obtain the best BER performance. Also, due to these simulation results it is possible to highlight the effect of the correlation over the BER performance which increases the bit-error rate. 


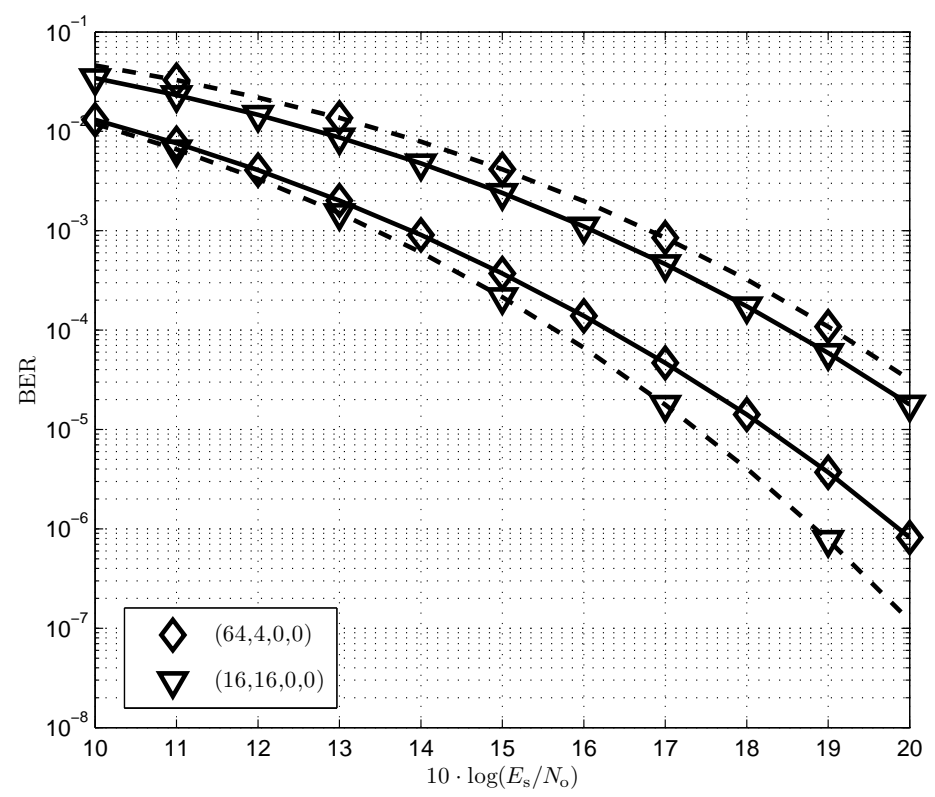

Figure 3-40: BER curves with GMD technique (dashed line) assuming perfect interference cancellation compared to BER curves with SVD (solid line) when using the transmission modes introduced in the legend with equal-SNR PA and transmitting $8 \mathrm{bit} / \mathrm{s} / \mathrm{Hz}$ over frequency non-selective $(4 \times 4)$ MIMO channels with antenna correlation

Furthermore, such as the core of this doctoral thesis chapter as Resources Allocation techniques, is including the optimal power allocation technique based on the Lagrange multiplier method, the suboptimal equal-SNR PA, the water-filling technique and the Iterative Bit and Power Allocation algorithm related to minimize the overall BER performance deeply studied. The accomplished computer simulations show the improvements made by these resource allocation techniques over the BER MIMO curves reducing the bit-error rate.

The opportunity to design and determine the number of active MIMO layers, the overall data rate, the QAM modulation schema per layer and per time slot and all combined with an appropriate resource allocation technique can remarkably improve the performance of a correlated MIMO communication system. In this work, a noteworthy BER performance improvement has been accomplished by using optimal bit and power allocation techniques based on iterative allocation methods over frequency non-selective $(4 \times 4)$ MIMO channels with and without correlation effect between 


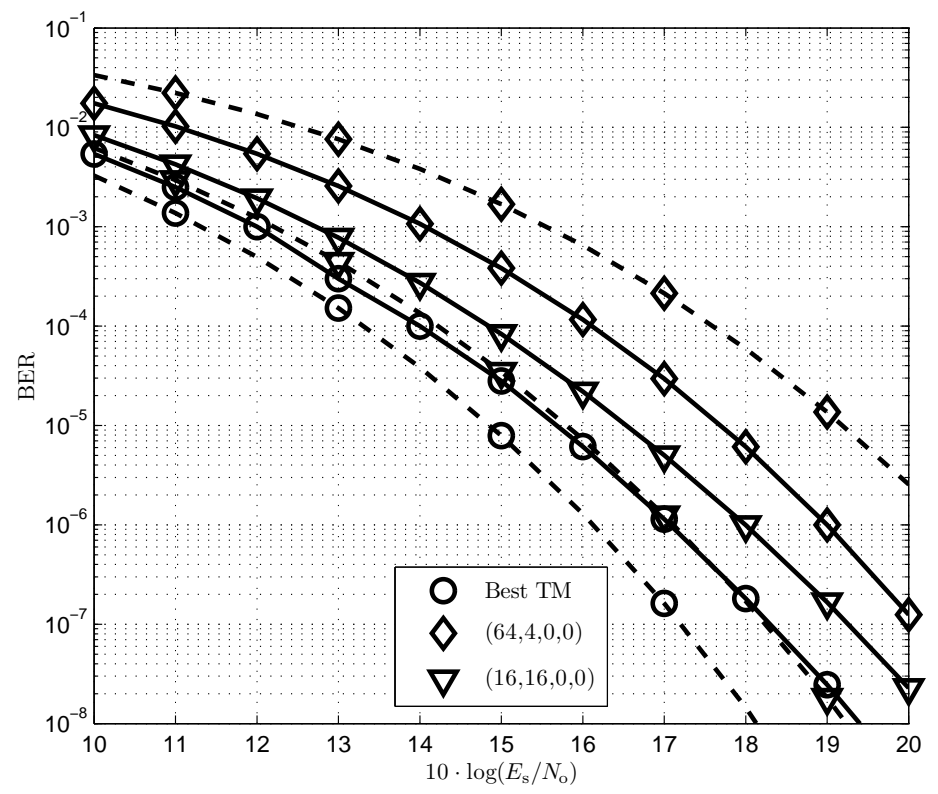

Figure 3-41: BER curves with GMD technique (dashed line) assuming perfect interference cancellation compared to BER curves with SVD (solid line) and the best transmission mode per each time slot with equal-SNR PA and transmitting $8 \mathrm{bit} / \mathrm{s} / \mathrm{Hz}$ over frequency non-selective $(4 \times 4)$ MIMO channels without antenna correlation

transmit antennas. Additionally, in order to develop these iterative power allocation algorithms (due to the transmit power is not uniformly distributed over active layers any longer), a new approach of suboptimal power allocation parameter $p_{\mathrm{IBPA}}^{(\mu, k)}$ has been perfected from the previous uniformly distributed power parameter $p^{(\mu, k)}$.

On the other hand, the performance obtained by the Singular Value Decomposition technique and the Geometric Mean Decomposition has been compared. Figure 3-39 demonstrates that the overall MIMO BER performance based on the GMD technique seems significant better than SVD technique when equal bit loading per active layer is used, i.e. $(16,16,0,0)$. By contrast, SVD-aided with equal-SNR PA technique presents a better performance when unequal bit allocation per layer is employed, i.e. $(64,4,0,0)$. The same conclusion can be applied for correlated MIMO systems according to Figure 3-40.

Finally, both decomposition techniques are compared taking into consideration the best transmission mode per time slot algorithm previously described. In order 


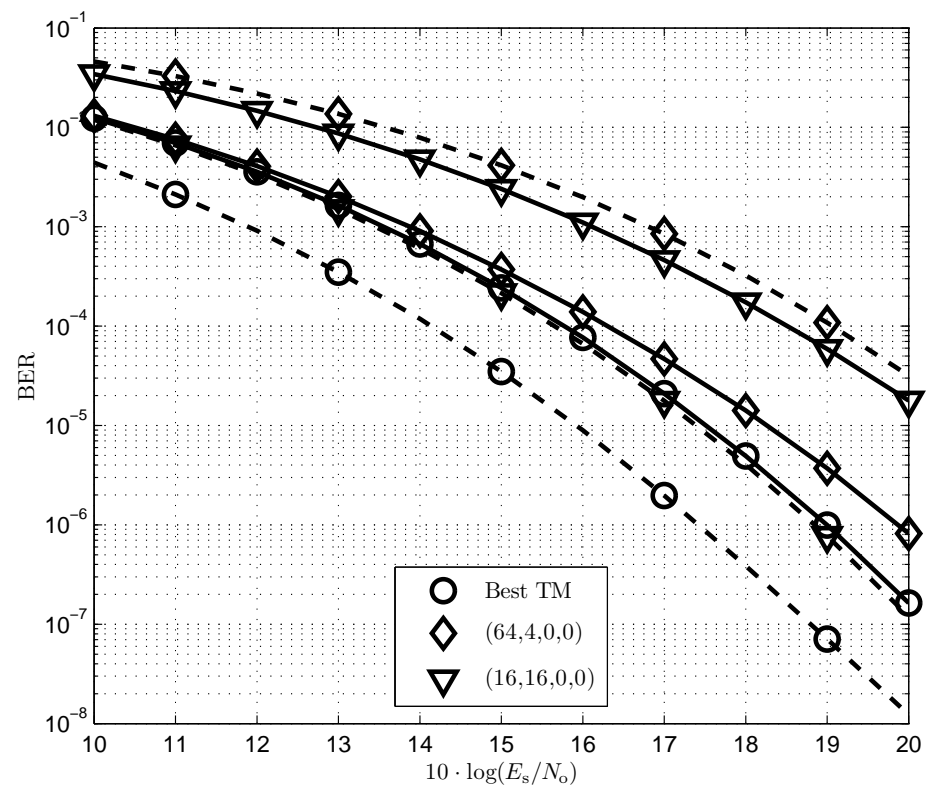

Figure 3-42: BER curves with GMD technique (dashed line) assuming perfect interference cancellation compared to BER curves with SVD (solid line) and the best transmission mode per each time slot with equal-SNR PA and transmitting $8 \mathrm{bit} / \mathrm{s} / \mathrm{Hz}$ over frequency non-selective $(4 \times 4)$ MIMO channels with antenna correlation

to find out the best bit allocation performance per time slot, it is required to find the most appropriate QAM constellation with the minimum transmit power. The results reveal that the GMD-aided MIMO systems present a remarkably better BER performance than the SVD-aided MIMO systems without correlation in Figure 3-41 and with correlation in Figure 3-42. 


\section{Chapter 4}

\section{Multi-user MIMO Systems}

\subsection{SVD-aided Multi-user MIMO System Model}

The multi-user MIMO (MU-MIMO) system model considered in this chapter consists of a single base station (BS) supporting $L_{\mathrm{MS}}$ mobile stations (MSs) [12].

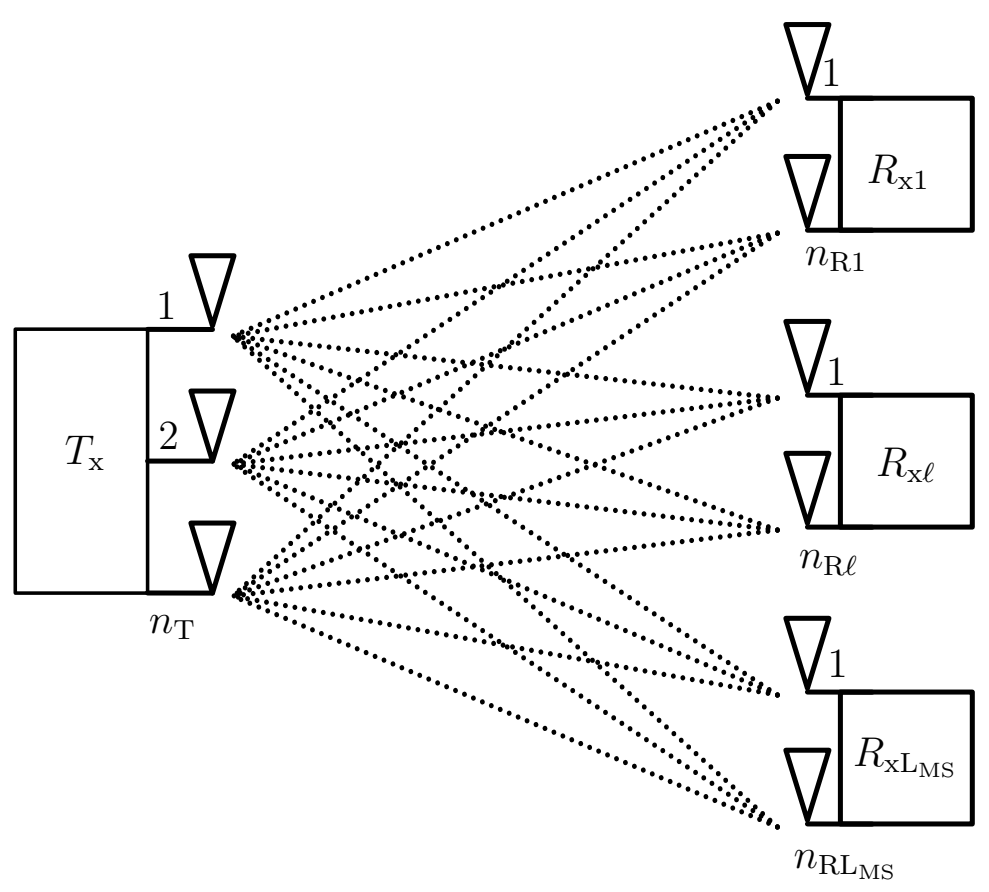

Figure 4-1: Multi-user MIMO system model

The BS is equipped with $n_{\mathrm{T}}$ transmit antennas, while the $\ell$-th (with $\ell=1, \ldots, L_{\mathrm{MS}}$ ) MS has $n_{\mathrm{R} \ell}$ receive antennas, i.e., the total number of receive antennas including all 
$L_{\mathrm{MS}}$ MSs is given by $n_{\mathrm{R}}=\sum_{\ell=1}^{L_{\mathrm{MS}}} n_{\mathrm{R} \ell}$. The $\left(n_{\mathrm{R} \ell} \times 1\right)$ user specific symbol vector $\mathbf{s}_{\ell}$ to be transmitted by the BS is given by

$$
\mathbf{s}_{\ell}=\left(s_{(\ell, 1)}, s_{(\ell, 2)}, \ldots, s_{\left(\ell, n_{R}\right)}\right)^{\mathrm{T}}
$$

where $\mathbf{s}=\sum_{\ell=1}^{L_{\mathrm{MS}}} s_{\ell}$

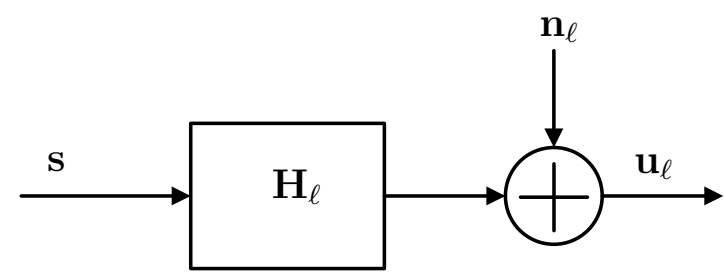

Figure 4-2: Multi-user MIMO system model

At the receiver side, according to the multi-user MIMO system model depicted in Figure 4 -2, the $\left(n_{\mathrm{R} \ell} \times 1\right)$ vector $\mathbf{u}_{\ell}$ of the $\ell$-th MS is given by

$$
\mathbf{u}_{\ell}=\mathbf{H}_{\ell} \cdot \mathbf{s}+\mathbf{n}_{\ell},
$$

and can be extended taking into consideration both multi-user and multi-antenna interfaces being expressed by the following equation

$$
\mathbf{u}_{\ell}=\mathbf{H}_{\ell} \cdot \mathbf{s}_{\ell}+\sum_{i=1, i \neq \ell}^{L_{\mathrm{MS}}} \mathbf{H}_{\ell} \cdot \mathbf{s}_{i}+\mathbf{n}_{\ell} .
$$

The $\left(n_{\mathrm{R} \ell} \times n_{\mathrm{T}}\right)$ channel matrix $\mathbf{H}_{\ell}$ connects the $n_{\mathrm{T}}$ BS specific transmit antennas with the $n_{\mathrm{R} \ell}$ receive antennas of the $\ell$-th MS. It is assumed that the coefficients of the channel matrix $\mathbf{H}_{\ell}$ are independent and Rayleigh distributed with equal variance. The interference between the different antenna's data streams, which is introduced by the off-diagonal elements of the channel matrix $\mathbf{H}_{\ell}$, requires appropriate signal processing strategies. A popular technique is based on the SVD of the system matrix $\mathbf{H}_{\ell}$. Upon carrying out the SVD of $\mathbf{H}_{\ell}$ with $n_{\mathrm{T}} \geq n_{\mathrm{R}}$ and assuming that the rank of the matrix $\mathbf{H}_{\ell}$ equals $n_{\mathrm{R} \ell}$, i.e., $\operatorname{rank}\left(\mathbf{H}_{\ell}\right)=n_{\mathrm{R} \ell}$, is obtained 


$$
\mathbf{H}_{\ell}=\mathbf{U}_{\ell} \cdot \mathbf{V}_{\ell} \cdot \mathbf{D}_{\ell}^{\mathrm{H}},
$$

where the $\left(n_{\mathrm{R} \ell} \times n_{\mathrm{R} \ell}\right)$ matrix $\mathbf{U}_{\ell}$ and the $\left(n_{\mathrm{T}} \times n_{\mathrm{T}}\right)$ matrix $\mathbf{D}_{\ell}^{\mathrm{H}}$ are unitary ones. In order to remove the multi-antenna and multi-user interferences perfectly, firstly, the $\left(n_{\mathrm{R} \ell} \times n_{\mathrm{T}}\right)$ diagonal matrix $\mathbf{V}_{\ell}$ can be decomposed into a matrix $\mathbf{V}_{\ell \mathrm{u}}$ containing the non-zero eigenvalues of $\mathbf{H}_{\ell}^{\mathrm{H}} \cdot \mathbf{H}_{\ell}$, i.e.,

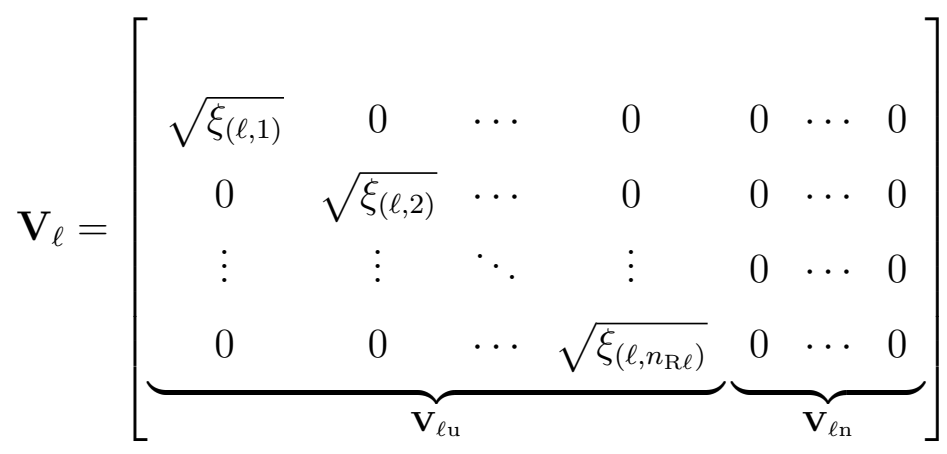

the decomposition of the matrix $\mathbf{V}_{\ell}$ results in

$$
\mathbf{V}_{\ell}=\left(\mathbf{V}_{\ell \mathrm{u}} \mathbf{V}_{\ell \mathrm{n}}\right)=\left(\mathbf{V}_{\ell \mathrm{u}} 0\right)
$$

Additionally, the $\left(n_{\mathrm{T}} \times n_{\mathrm{T}}\right)$ unitary matrix $\mathbf{D}_{\ell}$ can be decomposed into a $\left(n_{\mathrm{T}} \times n_{\mathrm{R} \ell}\right)$ matrix $\mathbf{D}_{\ell u}$ constituted by the eigenvectors corresponding to the non-zero eigenvalues of $\mathbf{H}_{\ell}^{\mathrm{H}} \cdot \mathbf{H}_{\ell}$ and a $\left(n_{\mathrm{T}} \times\left(n_{\mathrm{T}}-n_{\mathrm{R} \ell}\right)\right)$ matrix $\mathbf{D}_{\ell \mathrm{n}}$ constituted by the eigenvectors corresponding to the zero eigenvalues of $\mathbf{H}_{\ell}^{\mathrm{H}} \cdot \mathbf{H}_{\ell}$. The decomposition of the matrix $\mathbf{D}_{\ell}^{\mathrm{H}}$ results in

$$
\mathbf{D}_{\ell}^{\mathrm{H}}=\left(\begin{array}{c}
\mathbf{D}_{\ell \mathrm{u}}^{\mathrm{H}} \\
\mathbf{D}_{\ell \mathrm{n}}^{\mathrm{H}}
\end{array}\right) .
$$

The resulting user-specific system model after the signal processing approach in order to remove multi-antenna interference by SVD technique is depicted in Figure 43.

On the other hand, in order to suppress multi-user interferences perfectly additional signal processing is required. The zero-forcing ( $\mathrm{ZF}$ ) preprocessing model is 


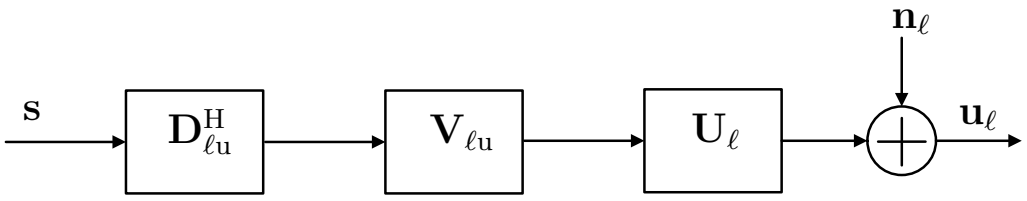

Figure 4-3: Multi-user MIMO system model after SVD-decomposition

depicted in Figure 4-4. MU-MIMO system is transformed into independent, noninterfering layers having unequal gains.

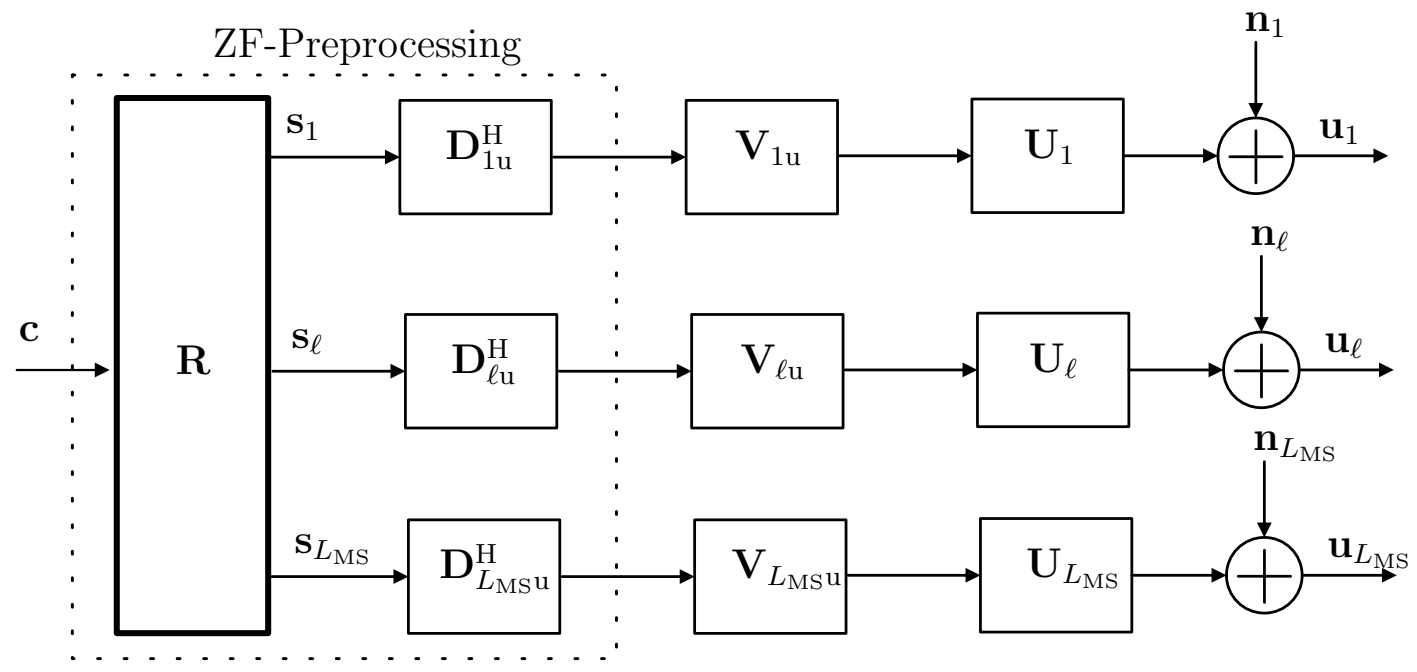

Figure 4-4: Multi-user MIMO system model with ZF-preprocessing

The vector $\mathbf{c}$ is preprocessed before its transmission by multiplying it with the Downlink (DL) preprocessing matrix R. After DL transmitter preprocessing, the $n_{\mathrm{T}}$-component signal $\mathbf{s}$ transmitted by the BS to the $L_{\mathrm{MS}}$ MSs results in

$$
\mathbf{s}=\sum_{\ell=1}^{L_{\mathrm{MS}}} \mathbf{s}_{\ell}=\mathbf{R} \cdot \mathbf{c}
$$

Finally, the received downlink signal $\mathbf{u}_{\ell}$ of the $\ell$-th MS may be expressed as

$$
\mathbf{u}_{\ell}=\mathbf{U}_{\ell} \cdot \mathbf{V}_{\ell \mathrm{u}} \cdot \mathbf{D}_{\ell \mathrm{u}}^{\mathrm{H}} \cdot \mathbf{R} \cdot \mathbf{c}+\mathbf{n}_{\ell}
$$

Taking all MSs received DL signals $\mathbf{u}_{\ell}$ into account, the $\left(n_{\mathrm{R}} \times 1\right)$ receive vector results in

$$
\mathbf{u}=\left(\mathbf{u}_{1}^{\mathrm{T}}, \mathbf{u}_{2}^{\mathrm{T}}, \ldots, \mathbf{u}_{L_{\mathrm{MS}}}^{\mathrm{T}}\right)^{\mathrm{T}}
$$


Then, the overall DL signal vector $\mathbf{u}$ including the received signals of all $L_{\mathrm{MS}} \mathrm{MSs}$ can be expressed by

$$
\mathbf{u}=\mathbf{U} \cdot \mathbf{V}_{\mathrm{u}} \cdot \mathbf{D}_{\mathrm{u}}^{\mathrm{H}} \cdot \mathbf{R} \cdot \mathbf{c}+\mathbf{n}
$$

with the overall $\left(n_{\mathrm{R}} \times n_{\mathrm{R}}\right)$ noise vector

$$
\mathbf{n}=\left(\mathbf{n}_{1}^{\mathrm{T}}, \mathbf{n}_{2}^{\mathrm{T}}, \ldots, \mathbf{n}_{L_{\mathrm{MS}}}^{\mathrm{T}}\right)^{\mathrm{T}}
$$

the $\left(n_{\mathrm{R}} \times n_{\mathrm{R}}\right)$ block diagonal matrix $\mathbf{U}$

$$
\mathbf{U}=\left[\begin{array}{cccc}
\mathbf{U}_{1} & 0 & \cdots & 0 \\
0 & \mathbf{U}_{2} & \cdots & 0 \\
\vdots & \vdots & \ddots & \vdots \\
0 & 0 & \cdots & \mathbf{U}_{L_{\mathrm{MS}}}
\end{array}\right]
$$

the $\left(n_{\mathrm{R}} \times n_{\mathrm{R}}\right)$ block diagonal matrix $\mathbf{V}_{\mathrm{u}}$

$$
\mathbf{V}_{\mathrm{u}}=\left[\begin{array}{cccc}
\mathbf{V}_{1 \mathrm{u}} & 0 & \cdots & 0 \\
0 & \mathbf{V}_{2 \mathrm{u}} & \cdots & 0 \\
\vdots & \vdots & \ddots & \vdots \\
0 & 0 & \cdots & \mathbf{V}_{L_{\mathrm{MS}} \mathrm{u}}
\end{array}\right]
$$

and the $\left(n_{\mathrm{T}} \times n_{\mathrm{R}}\right)$ matrix $\mathbf{D}_{\mathrm{u}}$ which is given by

$$
\mathbf{D}_{\mathrm{u}}=\left(\mathbf{D}_{1 \mathrm{u}}, \mathbf{D}_{2 \mathrm{u}}, \ldots, \mathbf{D}_{L_{\mathrm{MS}} \mathrm{u}}\right)
$$

In order to suppress the DL multi-user interfaces (MUI) efficiently, the DL preprocessing matrix $\mathbf{R}$ has to be designed to satisfy the following condition

$$
\mathbf{D}_{\mathrm{u}}^{\mathrm{H}} \cdot \mathbf{R}=\mathbf{P}_{\mathrm{G}}
$$

given the real-valued diagonal matrix $\mathbf{P}_{\mathrm{G}}$ takes the transmit-power constraint into account. In order to satisfy the previous formula, $\mathbf{R}$ can be defined as follows 


$$
\mathbf{R}=\mathbf{D}_{\mathrm{u}} \cdot\left(\mathbf{D}_{\mathrm{u}}^{\mathrm{H}} \cdot \mathbf{D}_{\mathrm{u}}\right)^{-1} \cdot \mathbf{P}_{\mathrm{G}}
$$

Taking into consideration the ZF design criterion for the DL preprocessing matrix into account shown in Figure 4-4, the matrix $\mathbf{P}_{\mathrm{G}}$ simplifies to a diagonal matrix, i.e. $\mathbf{P}_{\mathrm{G}}=\sqrt{\beta} \cdot \mathbf{I}_{\left(n_{\mathrm{R}} \times n_{\mathrm{R}}\right)}$, with the parameter $\sqrt{\beta}$ taking the transmit-power constraint into account. When taking the DL preprocessing matrix, defined in the formula below, into account, the overall received vector for all $L_{\mathrm{MS}}$ MSs can be simplified to

$$
\mathbf{u}=\mathbf{U} \cdot \mathbf{V}_{\mathrm{u}} \cdot \mathbf{P}_{\mathrm{G}} \cdot \mathbf{c}+\mathbf{n}
$$

Therein, the $\left(n_{\mathrm{R}} \times n_{\mathrm{R}}\right)$ block diagonal matrix $\mathbf{P}_{\mathrm{G}}$ is given by

$$
\mathbf{P}_{\mathrm{G}}=\left[\begin{array}{cccc}
\mathbf{P}_{\mathrm{G} 1} & 0 & \cdots & 0 \\
0 & \mathbf{P}_{\mathrm{G} 2} & \cdots & 0 \\
\vdots & \vdots & \ddots & \vdots \\
0 & 0 & \cdots & \mathbf{P}_{\mathrm{G} L_{\mathrm{MS}}}
\end{array}\right]
$$

In the general formula, the user-specific vector $\mathbf{u}_{\ell}$ can be expressed as

$$
\mathbf{u}_{\ell}=\mathbf{U}_{\ell} \cdot \mathbf{V}_{\ell \mathrm{u}} \cdot \mathbf{P}_{\mathrm{G} \ell} \cdot \mathbf{c}_{\ell}+\mathbf{n}_{\ell}
$$

with the user-specific $\left(n_{\mathrm{R} \ell} \times n_{\mathrm{R} \ell}\right)$ power allocation matrix

$$
\mathbf{P}_{\mathrm{G} \ell}=\left[\begin{array}{cccc}
\sqrt{p_{\mathrm{G}}^{(\ell, 1)}} & 0 & \cdots & 0 \\
0 & \sqrt{p_{\mathrm{G}}^{(\ell, 2)}} & \cdots & 0 \\
\vdots & \vdots & \ddots & \vdots \\
0 & 0 & \cdots & \sqrt{p_{\mathrm{G}}^{\left(\ell, n_{\mathrm{R} \ell}\right)}}
\end{array}\right]
$$

As long as the transmit power is uniformly distributed over the number of ac-

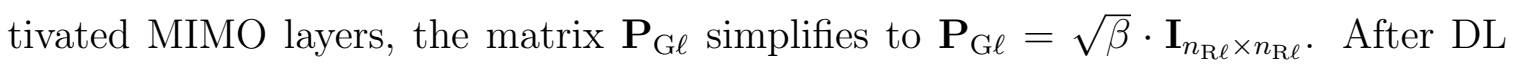
receiver side post-processing of the received signal vector $\mathbf{u}_{\ell}$ with the corresponding unitary matrix $\mathbf{U}_{\ell}^{\mathrm{H}}$, the user-specific decision variables result with $\mathbf{U}_{\ell}^{\mathrm{H}} \cdot \mathbf{n}_{\ell}=\mathbf{w}_{\ell}$ in 


$$
\mathbf{y}_{\ell}=\mathbf{U}_{\ell}^{\mathrm{H}} \cdot \mathbf{u}_{\ell}=\mathbf{V}_{\ell \mathrm{u}} \cdot \mathbf{P}_{\mathrm{G} \ell} \cdot \mathbf{c}_{\ell}+\mathbf{w}_{\ell}
$$

or alternatively with $\mathbf{U}^{\mathrm{H}} \mathbf{n}=\mathbf{w}$ in

$$
\mathbf{y}=\mathbf{U}^{\mathrm{H}} \cdot \mathbf{u}=\mathbf{V}_{\mathrm{u}} \cdot \mathbf{P}_{\mathrm{G}} \cdot \mathbf{c}+\mathbf{w}
$$

where interferences between the different antenna data streams as well as MUI imposed by the other users are avoided.

Simulation The simulation parameters of the analysed two-users MIMO system are listed on Table 4.1.

Table 4.1: Simulation parameters for multi-user MIMO system

\begin{tabular}{cccc}
\hline Transmit Power & BS Transmit Antennas & MS Receive Antennas & $L_{\text {MS }}$ mobile stations \\
\hline $1 W$ & 8 & 4 & 2 \\
\hline
\end{tabular}

The obtained user-specific BER curves are depicted in Figure 4-5 and Figure 4-6 according to different correlated scenarios. Figures depict the BER curves for the different QAM constellation sizes listed on Table 3.1.

Assuming a uniform distribution of the transmit power over the number of activated MIMO layers, it still turns out that not all MIMO layers have to be activated in order to achieve the best BERs. 


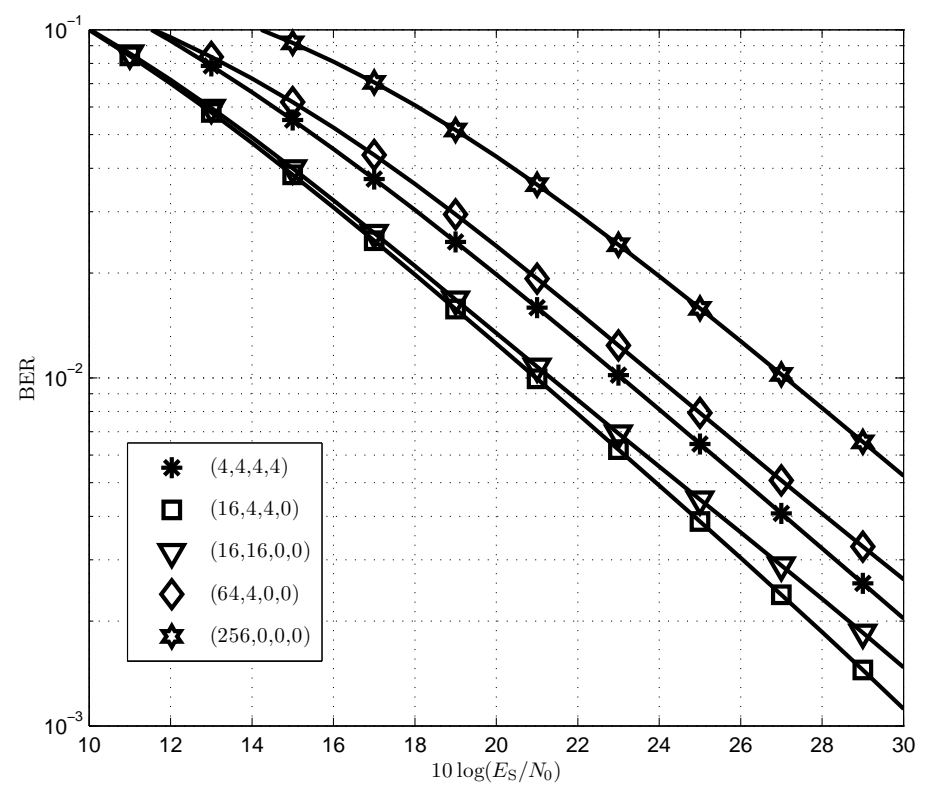

Figure 4-5: User-specific BERs comparison when using the transmission modes introduced in Table 3.1 and transmitting 8 bit/s/Hz over frequency non-selective uncorrelated SVD-aided Multi-user MIMO system

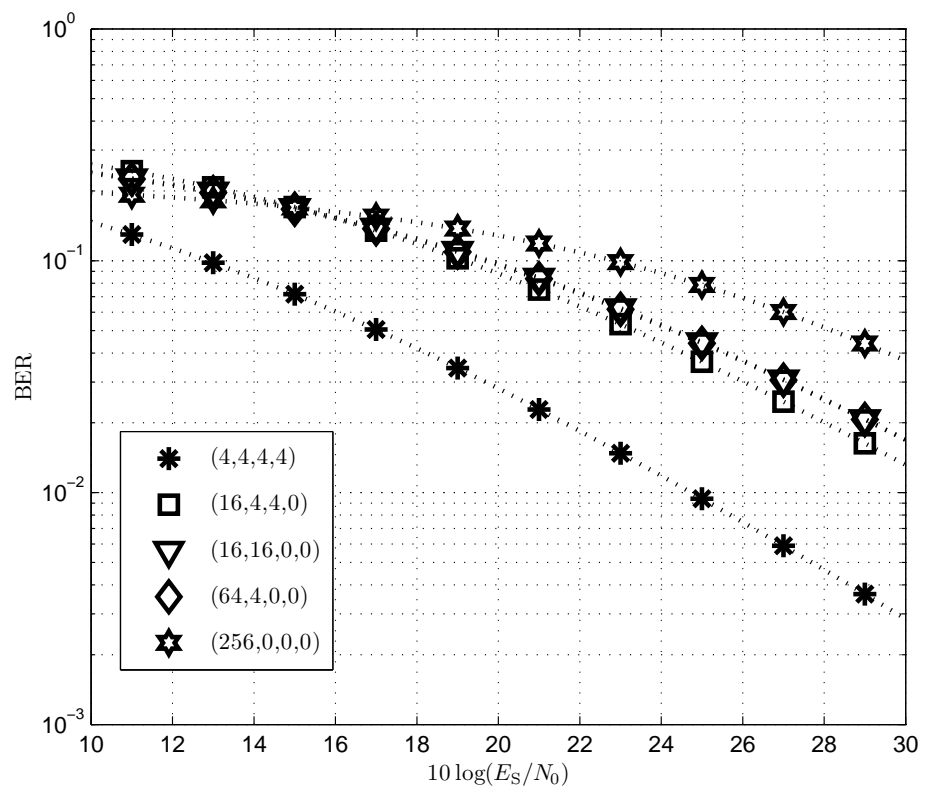

Figure 4-6: User-specific BERs comparison when using the transmission modes introduced in Table 3.1 and transmitting $8 \mathrm{bit} / \mathrm{s} / \mathrm{Hz}$ over frequency non-selective correlated SVD-aided Multi-user MIMO system 


\subsection{GMD-aided Multi-User MIMO System Model}

The system model considered in Figure 4-1 and Figure 4-2 for multi-user SVD-aided MIMO system is taken into account. Multi-user GMD-aided MIMO system is also described. The GMD decomposition technique is used trying to optimize the performance of MIMO systems. Applying the GMD decomposition over the Multi-user channel matrix $\mathbf{H}_{\ell}$ is simplified to

$$
\mathbf{H}_{\ell}=\mathbf{Q}_{\ell} \cdot \Sigma_{\ell} \cdot \mathbf{P}_{\ell}^{\mathrm{H}}
$$

where the $\left(n_{\mathrm{R} \ell} \times n_{\mathrm{R} \ell}\right)$ matrix $\mathbf{Q}_{\ell}$ and the $\left(n_{\mathrm{T}} \times n_{\mathrm{T}}\right)$ matrix $\mathbf{P}_{\ell}^{\mathrm{H}}$ are unitary ones. In order to remove the multi-antenna and multi-user interferences perfectly, firstly, the $\left(n_{\mathrm{R} \ell} \times n_{\mathrm{T}}\right)$ upper triangular matrix $\boldsymbol{\Sigma}_{\ell}$ can be decomposed by using pre- and post-processing. Secondly, in order to suppress multi-user interferences perfectly, additional signal processing is required.

In order to understand easily the effect produced by this MU-GMD technique, a no correlated multi-user GMD-aided MIMO channel with 8 BS transmit antennas, 4 MS receive antennas and $L_{\mathrm{MS}}=2$ mobile stations are analysed. The user specific real upper triangular matrix $\Sigma$ with $n_{\mathrm{R} \ell}=4$ active layers is mathematical described as follows

$$
\mathbf{H}_{\ell=1,2}^{(8 \times 8)}=\left(\begin{array}{llllllll}
0,403 & 0,253 & 0,454 & 0,765 & 0,072 & 0,001 & 0,341 & 0,135 \\
0,631 & 0,286 & 0,287 & 1,327 & 0,200 & 0,112 & 1,141 & 0,106 \\
0,773 & 0,093 & 0,213 & 1,080 & 0,540 & 0,628 & 1,360 & 0,356 \\
0,957 & 0,774 & 1,048 & 0,638 & 0,016 & 0,235 & 0,303 & 0,005 \\
0,562 & 1,726 & 0,124 & 0,248 & 1,235 & 0,302 & 0,859 & 1,356 \\
0,306 & 1,183 & 0,429 & 0,903 & 0,280 & 0,080 & 1,166 & 0,078 \\
1,208 & 2,198 & 0,371 & 0,128 & 0,559 & 0,830 & 0,362 & 0,818 \\
0,532 & 0,898 & 0,750 & 0,905 & 0,128 & 0,401 & 0,520 & 1,023
\end{array}\right)
$$




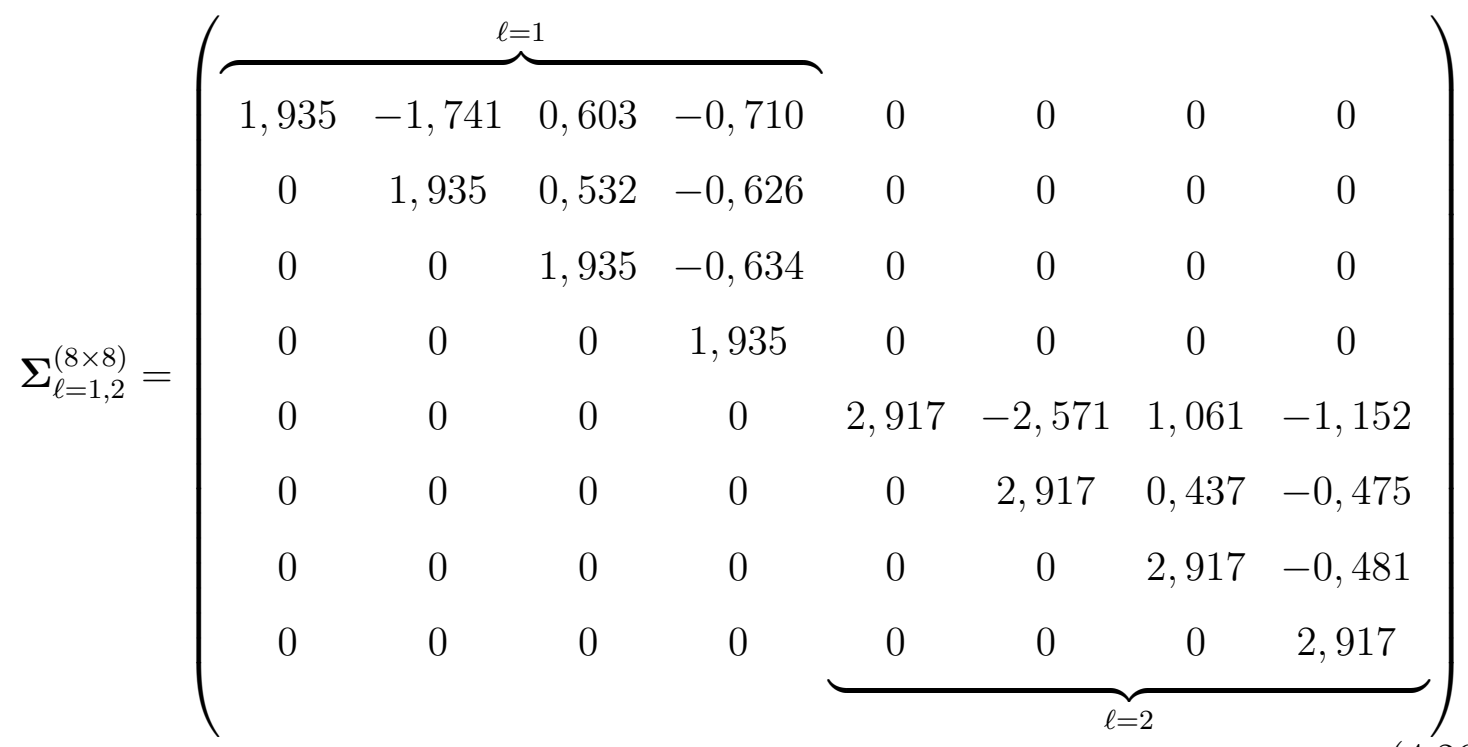

The ZF-preprocessing model is depicted in Figure 4-7. The MU-MIMO system is transformed into independent, non-interfering layers having user-specific equal gains. Perfect Interference Cancellation is also assumed.

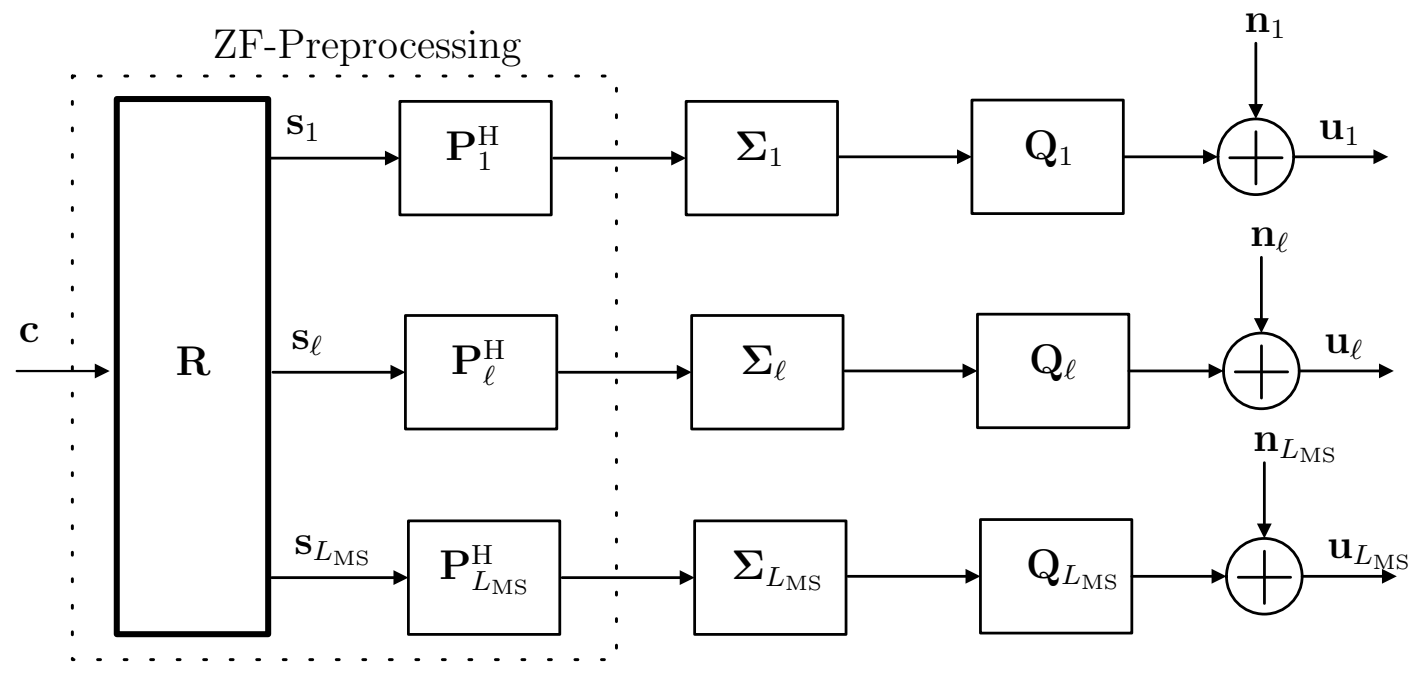

Figure 4-7: MU-MIMO system model with ZF-preprocessing

The vector $\mathbf{c}$ is preprocessed before its transmission by multiplying it with the DL preprocessing matrix R. After DL transmitter preprocessing, the $n_{\mathrm{T}}$-component signal s transmitted by the $\mathrm{BS}$ to the $L_{\mathrm{MS}} \mathrm{MSs}$ results in 


$$
\mathbf{s}=\sum_{\ell=1}^{L_{\mathrm{MS}}} \mathbf{s}_{\ell}=\mathbf{R} \cdot \mathbf{c}
$$

Finally, the received downlink signal $\mathbf{u}_{\ell}$ of the $\ell$-th MS may be expressed as

$$
\mathbf{u}_{\ell}=\mathbf{Q}_{\ell} \cdot \Sigma_{\ell} \cdot \mathbf{P}_{\ell}^{\mathrm{H}} \cdot \mathbf{R} \cdot \mathbf{c}+\mathbf{n}_{\ell}
$$

In order to suppress the DL multi-user interfaces (MUI) efficiently, the DL preprocessing matrix $\mathbf{R}$ has to be designed to satisfy the following condition

$$
\mathbf{P}^{\mathrm{H}} \cdot \mathbf{R}=\mathbf{P}_{\mathrm{G}},
$$

given the real-valued diagonal matrix $\mathbf{P}_{\mathrm{G}}$ takes the transmit-power constraint into account. In order to satisfy the previous formula, $\mathbf{R}$ can be defined as follows

$$
\mathbf{R}=\mathbf{P} \cdot\left(\mathbf{P}^{\mathrm{H}} \cdot \mathbf{P}\right)^{-1} \cdot \mathbf{P}_{\mathrm{G}}
$$

Taking into consideration the ZF design criterion for the DL preprocessing matrix into account shown in Figure 4-7, the matrix $\mathbf{P}_{\mathrm{G}}$ simplifies to a diagonal matrix, i.e. $\mathbf{P}_{\mathrm{G}}=\sqrt{\beta} \cdot \mathbf{I}_{\left(n_{\mathrm{R}} \times n_{\mathrm{R}}\right)}$, with the parameter $\sqrt{\beta}$ taking the transmit-power constraint into account. When taking the DL preprocessing matrix, defined in the formula below, into account, the overall received vector for all $L_{\mathrm{MS}} \mathrm{MSs}$ can be simplified to

$$
\mathbf{u}=\mathbf{Q} \cdot \mathbf{\Sigma} \cdot \mathbf{P}_{\mathrm{G}} \cdot \mathbf{c}+\mathbf{n}
$$

After DL receiver side post-processing of the received signal vector $\mathbf{u}$ with the corresponding unitary matrix $\mathbf{Q}^{\mathrm{H}}$, the decision variables result with $\mathbf{Q}^{\mathrm{H}} \mathbf{n}=\mathbf{w}$ in

$$
\mathbf{y}=\mathbf{Q}^{\mathrm{H}} \cdot \mathbf{u}=\mathbf{\Sigma} \cdot \mathbf{P}_{\mathrm{G}} \cdot \mathbf{c}+\mathbf{w}
$$

where interferences between the different antenna data streams as well as MUI imposed by the other users are perfectly avoided. 
Simulation The simulation parameters of the analysed two-users MIMO system are listed on Table 4.1. The obtained user-specific BER curves are depicted in Figure 4-8 and Figure 4-9 according to different correlated scenarios. Figures depict the BER curves for the different QAM constellation sizes listed on Table 3.1.

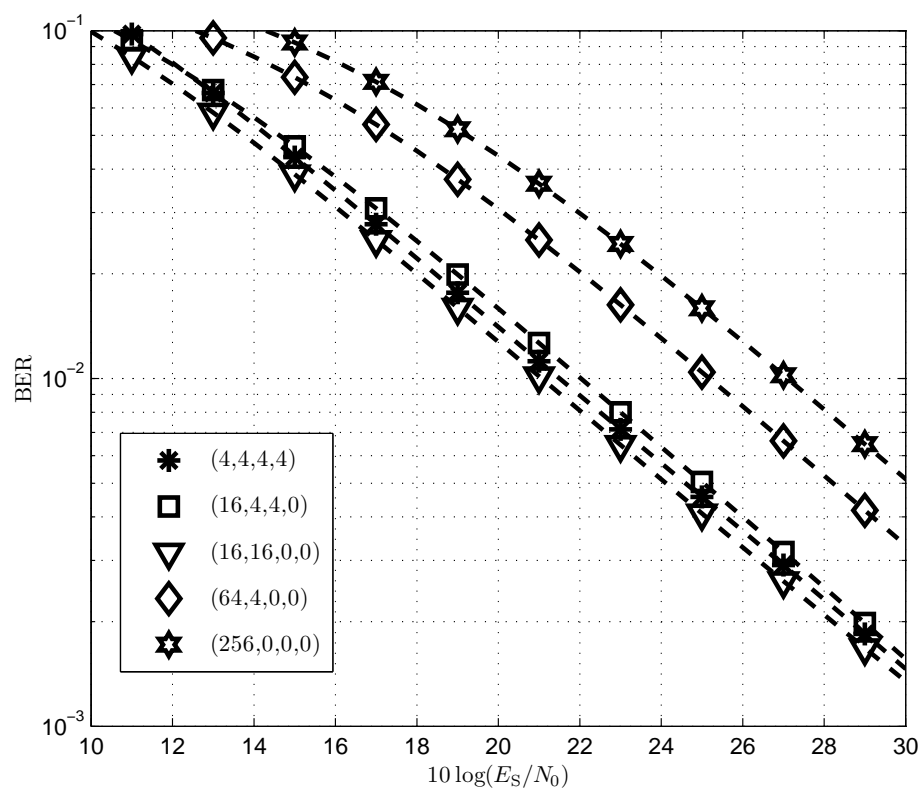

Figure 4-8: User-specific BERs comparison when using the transmission modes introduced in Table 3.1 and transmitting $8 \mathrm{bit} / \mathrm{s} / \mathrm{Hz}$ over frequency non-selective uncorrelated GMD-aided MU-MIMO systems 


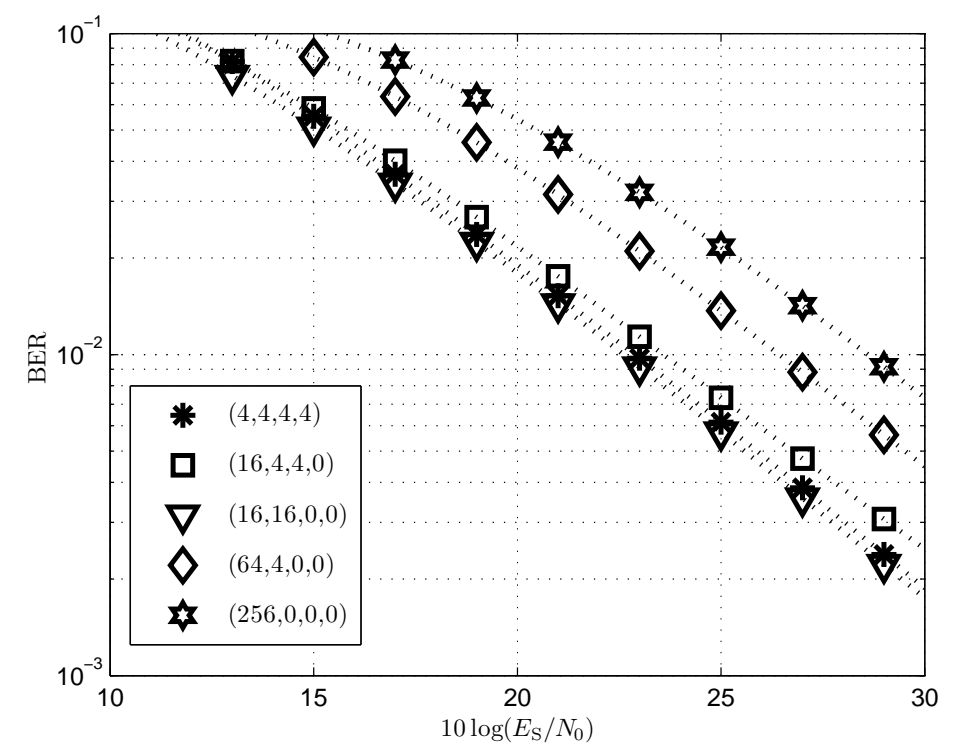

Figure 4-9: User-specific BERs comparison when using the transmission modes introduced in Table 3.1 and transmitting $8 \mathrm{bit} / \mathrm{s} / \mathrm{Hz}$ over frequency non-selective correlated GMD-aided MU-MIMO systems 


\subsection{Chapter Conclusions}

In this fourth chapter of the doctoral thesis, the multi-user MIMO scheme aided by two different mathematical decomposition techniques (SVD and GMD) has been analysed. The proposed downlink system includes a single base station supporting two mobile stations. The choice of the number of bits per symbol and the number of MIMO layers combined with proper pre-and post-processing over the transmit signal substantially affects the performance of the frequency non-selective MU-MIMO system, suggesting that not all MIMO layers have to be activated in order to achieve the best BER per user-specific performance.

Furthermore, a comparison between SVD-aided MU-MIMO and GMD-aided MUMIMO systems has been carried out. The obtained user-specific BER curves comparison is depicted for two different specific QAM constellation sizes, (64, 4, 0,0) and $(16,16,0,0)$. Figure 4-10 shows that the overall MIMO BER performance based on the GMD technique seems briefly better than the SVD technique when using equal bit loading per active layer. By contrast, SVD-aided technique presents better performance when unequal bit allocation per layer, e.g. $(64,4,0,0)$. The obtained computer simulations confirm the obtained results within the single-user system $(\ell=1)$ with a loss of BER due to $16 \mathrm{bit} / \mathrm{s} / \mathrm{Hz}$ that are sent in MU-MIMO compared to the 8 bit/s/Hz from SU-MIMO systems. 


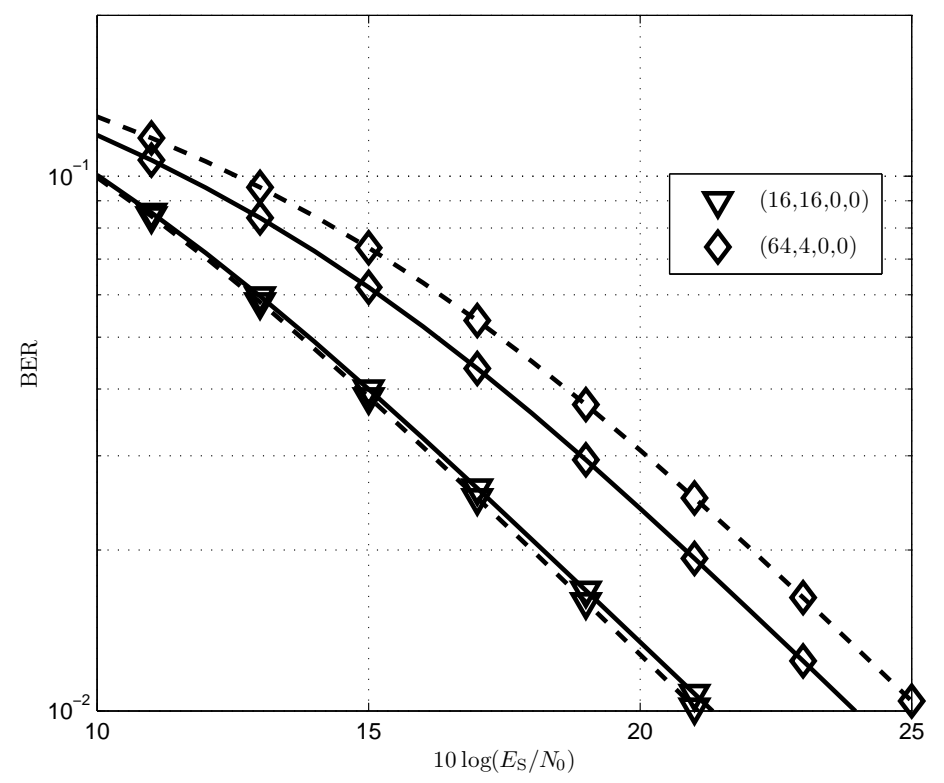

Figure 4-10: User-specific BERs comparison between the SVD-aided and GMD-aided MIMO system when using the transmission modes $(16,16,0,0)$ and $(4,4,4,4)$ and transmitting $8 \mathrm{bit} / \mathrm{s} / \mathrm{Hz}$ over frequency non-selective uncorrelated Rayleigh channels 


\section{Chapter 5}

\section{Conclusions}

\subsection{Achievements}

The main goal of this doctoral thesis is to characterize the correlation effect over frequency non-selective $(4 \times 4)$ MIMO systems performance under different spatial antennas distribution (linear and non-linear) and analyse different resource allocation strategies in order to minimize the overall bit-error rate performance.

In this doctoral thesis the following achievements have been addressed:

- The influence of the receiver-side antennas correlation over the bit-error rate in frequency non-selective $(4 \times 4)$ MIMO systems using CCDFs of the singular values has been characterized. According to the obtained results it is concluded that under the effect of correlation not necessarily that all layers might be used for the data transmission since the weighting of the stronger layer within the MIMO system becomes even stronger.

- Different resources (number of active MIMO layers, number of the overall data rate, QAM modulation schema per layer and per time slot data rate, bits-perlayer and power) allocation techniques has been analysed with the aim to increase the degree of design freedom, which largely affects the BER performance on correlated MIMO communication systems. Due to these Resource Allocation strategies developed in this doctoral thesis the overall BER performance 
has been improved.

- Optimal and suboptimal Power Allocation techniques have been compared. The obtained computer simulations demonstrate that the overall MIMO channel BER losses based on the equal layer quality criteria seem quite acceptable when using the proposed suboptimal bit loading algorithm.

- Novel optimal resources allocation techniques based on iterative allocation methods over frequency non-selective $(4 \times 4)$ MIMO channels with and without antenna's correlation have been developed. According to the simulation results, a noteworthy BER performance improvement has been accomplished. Additionally, in order to develop these iterative power allocation algorithms, a new approach of suboptimal power allocation parameter has been perfected for not uniformly distributed over active layers from the previous uniformly distributed power parameter.

- The Singular Value Decomposition and the Geometric Mean Decomposition techniques have been compared. From the obtained results it is observed that the overall MIMO BER performance based on the GMD technique seems significantly better than the SVD technique when the best transmission mode per time slot is taken into account.

- The single-user MIMO system has been extended to a multi-user MIMO system model, and the BER performance has been obtained. There is a loss of BER due to $16 \mathrm{bit} / \mathrm{s} / \mathrm{Hz}$ sent in MU-MIMO and compared to the $8 \mathrm{bit} / \mathrm{s} / \mathrm{Hz}$ from SU-MIMO systems.

- Multi-user and multi-antenna interferences using proper pre-and post-processing strategies over the transmit signal have been eliminated.

\subsection{Contributions}

- Journals 
- Ahrens, A., Benavente-Peces, C. and Cano-Broncano, F. "Power Allocation in SVD- and GMD-assisted MIMO Systems". Optimization and Engineering, 1-22, 07 August 2016. DOI 10.1007/s11081-016-9334-1

- Benavente-Peces C., Cano-Broncano F., Ahrens A., Ortega-Gonzalez F.J. and Pardo-Martn J.M., "Analysis of Singular Values PDF and CCDF on Receiver-side Antennas Correlated MIMO Channels". Electronics Letters, Volume 49, Issue 9, pp. 625-627, 25 April 2013. DOI: 10.1049/el.2013.0390

- Book Chapter

- Authors: Ahrens A., Cano-Broncano F., Benavente-Peces C. Editor: Obaidat, Mohammad S., Filipe J., Holzinger, A. Title: "Bit- and Power Allocation in GMD and SVD-Based MIMO Systems". Book Title: "e-Business and Telecommunications". Year: 2015 Publisher: "Springer Berlin Heidelberg" Pages: 499-517 ISBN: 978-3-319-25914-7 DOI: 10.1007/978-3-31925915-4-26

- Authors: Ahrens A., Cano-Broncano F., Benavente-Peces C. Editor: Obaidat, Mohammad S., Filipe J. Title: "Transmitter-Side Antennas Correlation in SVD-assisted MIMO Systems". Book Title: "e-Business and Telecommunications". Year: 2014 Publisher: "Springer Berlin Heidelberg" Pages: 402-417 ISBN: 978-3-662-44787-1 DOI: 10.1007/978-3-662-44788-824

- Authors: Aust S., Ahrens A., Cano-Broncano F., Benavente-Peces C. Editor: Obaidat, Mohammad S., Sevillano J., Filipe J. Title: "Downlink Multiuser MIMO-OFDM Systems". Book Title: "e-Business and Telecommunications". Year: 2012 Publisher: "Springer Berlin Heidelberg" Pages: 449-463 ISBN: 978-3-642-35755-8 DOI: 10.1007/978-3-642-35755-8-32

- Conferences

- Ahrens A., Cano-Broncano F., Benavente-Peces C., "Resource Allocation in GMD and SVD-based MIMO System" in 11th International Confer- 
ence on Wireless Information Networks and Systems (WINSYS), Vienna, Austria, 28-30 August 2014

- Cano-Broncano, F., Ahrens, A. and Benavente-Peces, C., "Iterative Bitand Power Allocation in Correlated MIMO Systems", International Conference on Pervasive and Embedded Computing and Communication Systems (PECCS), Lisbon, Portugal. 7-9 January, 2014

- Ahrens A., Cano-Broncano F., Benavente-Peces C., "Power Distribution for SVD-aided MIMO Transmission with Receiver-side Antennas Correlation" in 10th IASTED International Conference on Signal Processing, Pattern Recognition and Applications (SPPRA), Innsbruck, Austria, 1214 February, 2013

- Cano-Broncano F., Benavente-Peces C., Ahrens A., Ortega-Gonzalez F.J., Pardo-Martin J.M. "Analysis of MIMO Systems with Transmitter-side Antennas Correlation" in International Conference on Pervasive and Embedded Computing and Communication Systems (PECCS), Barcelona, Spain, 19-21 February, 2013

- Ahrens A., Cano-Broncano F., Benavente-Peces C., "Modulation-mode and Power Assignment in SVD-assisted MIMO Systems with Transmitterside Antennas Correlation" in 10th International Conference on Wireless Information Networks and Systems (WINSYS), Reykjavik, Iceland, 29-31 July 2013

- Cano-Broncano F., Ahrens A., Benavente-Peces C., "Adaptive Power Allocation in SVD-aided SDM-MIMO Systems" in XV International PhD Workshop OWD,pp 268-273, Wisla 19-22 October 2013

- Cano-Broncano F., Ahrens A., Benavente-Peces C., "Power Allocation Techniques in MIMO Systems" in XV International PhD Workshop OWD,pp 274-277, Wisla 19-22 October 2013

- Mamdouh El-Shafiey A., Cano-Broncano F., Ahrens A., "Bit and Power Allocation Strategies in SVD-equalized Broadband MIMO Systems" in 
XV International PhD Workshop OWD, pp 262-267, Wisla 19-22 October 2013.

- Cano-Broncano F., Benavente-Peces C., Ahrens A., Ortega-Gonzalez F.J, Pardo-Martin J.M, "Multi-user MIMO Systems with Antennas Correlation and Linear and Nonlinear Spatial Distribution", International Conference on Pervasive and Embedded Computing and Communication Systems (PECCS), Rome, 2012

- Benavente-Peces C., Cano-Broncano F., Aust S., Ahrens A. "Modulationmode Assigment for SVD-assisted Multi-user MIMO Systems with Correlation", International Conference on Pervasive and Embedded Computing and Communication System (PECCS), Vilamoura, Algarve, 2011 


\section{Appendix - Mathematical concepts}

Matrix Definition A matrix is simply a rectangular array of real numbers. A $(m \times n)$ matrix is an array having $m$ rows and $n$ columns, such as [13]

$$
\mathbf{A}=\left(\begin{array}{cccc}
a^{(11)} & a^{(12)} & \ldots & a^{(1 n)} \\
a^{(21)} & a^{(22)} & \ldots & a^{(2 n)} \\
\vdots & \vdots & \ddots & \vdots \\
a^{(m 1)} & a^{(m 2)} & \ldots & a^{(m n)}
\end{array}\right)
$$

vec Operator The vec operator creates a column vector from a matrix $\mathbf{A}$ by stacking the column vectors of $\mathbf{A}=\left[a^{(1)} a^{(2)} \ldots a^{(n)}\right]$ below itself:

$$
\operatorname{vec}(\mathbf{A})=\left[\begin{array}{c}
a^{(1)} \\
a^{(2)} \\
\vdots \\
a^{(n)}
\end{array}\right]
$$

Kronecker Product The Kronecker Product of two matrices $\mathbf{A}(m \times n)$ and $\mathbf{B}$ $(p \times q)$ is defined to be the $(m p \times n q)$ matrix [31]

$$
\mathbf{A} \otimes \mathbf{B}=\left[\begin{array}{cccc}
a^{(11)} \mathbf{B} & a^{(12)} \mathbf{B} & \cdots & a^{(1 p)} \mathbf{B} \\
a^{(21)} \mathbf{B} & a^{(22)} \mathbf{B} & \cdots & a^{(2 p)} \mathbf{B} \\
\vdots & \vdots & \vdots & \vdots \\
a^{(n 1)} \mathbf{B} & a^{(n 2)} \mathbf{B} & \cdots & a^{(n p)} \mathbf{B}
\end{array}\right]
$$




\section{Properties}

$$
\begin{gathered}
\mathbf{A} \otimes(\mathbf{B} \otimes \mathbf{C})=(\mathbf{A} \otimes \mathbf{B}) \otimes C \\
(\mathbf{A} \otimes \mathbf{B})(\mathbf{C} \otimes \mathbf{D})=\mathbf{A} \cdot \mathbf{C} \otimes \mathbf{B} \cdot \mathbf{D}(\text { if } \mathbf{A} \cdot \mathbf{C} \text { and } \mathbf{B} \cdot \mathbf{D} \text { exist }) \\
(\mathbf{A} \otimes \mathbf{B})^{T}=\mathbf{A}^{T} \otimes \mathbf{B}^{T}
\end{gathered}
$$

Eigenvalues and Eigenvectors For an $(n \times n)$ matrix $\mathbf{A}$, scalars $\lambda_{\mathrm{s}}$ and vectors $\mathbf{x}^{(n \times 1)} \neq 0$ satisfying $\mathbf{A} \mathbf{x}=\lambda_{\mathrm{s}} \mathbf{x}$ are called eigenvalues and eigenvectors of $\mathbf{A}$, respectively, and any such pair $\left(\lambda_{\mathrm{s}}, \mathbf{x}\right)$, is called an eigenpair for $\mathbf{A}$. The set of distinct eigenvalues, denoted by $\sigma(\mathbf{A})$, is called the spectrum of $\mathbf{A}$ [31].

- $\lambda_{\mathrm{s}} \in \sigma(\mathbf{A}) \Longleftrightarrow \mathbf{A}-\lambda_{\mathrm{s}} \mathbf{I}$ is singular $\Longleftrightarrow \operatorname{det}\left(\mathbf{A}-\lambda_{\mathrm{s}} \mathbf{I}\right)=0$.

- $\left\{\mathbf{x} \neq 0 \mid \mathbf{x} \in N\left(\mathbf{A}-\lambda_{\mathrm{s}} \mathbf{I}\right)\right\}$ is the set of all eigenvectors associated with $\lambda_{\mathrm{s}}$. From now on, $N\left(\mathbf{A}-\lambda_{\mathrm{s}} \mathbf{I}\right)$ is called an eigenspace for $\mathbf{A}$

- Nonzero row vectors $\mathbf{y}^{*}$ such that $\mathbf{y}^{*}\left(\mathbf{A}-\lambda_{\mathbf{s}} \mathbf{I}\right)=0$ are called lefthand eigenvectors for $\mathbf{A}$.

The Exponential of a Matrix Suppose $\mathbf{A} \in \Re^{(n \times n)}$ can be diagonalized, say $\mathbf{A}=\mathbf{E} \cdot \mathbf{D} \cdot \mathbf{E}^{-1}$. Then it is observed for any $k>0, \mathbf{A}^{k}=\mathbf{E} \cdot \mathbf{D}^{k} \cdot \mathbf{E}^{-1}$. In particular it is possible to make three statements [31].

- if $\mathbf{A}$ is a diagonalizable real matrix with non negative eigenvalues, then $\mathbf{A}$ has a square root; in fact $k$-th roots of $\mathbf{A}$ for all positive integers $k$ are given by

$$
\mathbf{A}^{\frac{1}{k}}=\mathbf{E} \cdot \mathbf{D}^{\frac{1}{k}} \cdot \mathbf{E}^{-1}
$$

- if $\mathbf{A}$ is diagonalizable and none of the eigenvalues of $\mathbf{A}$ are 0 , then the negative powers of $\mathbf{A}$ are found from the formula 


$$
\mathbf{A}^{-k}=\mathbf{E} \cdot \mathbf{D}^{-k} \cdot \mathbf{E}^{-1}
$$

here $\mathbf{A}^{-k}$ means $\left(A^{-1}\right)^{k}$.

- if all the eigenvalues $\lambda_{\mathrm{s}}$ of $\mathbf{A}$ satisfy $0 \leq \lambda_{\mathrm{s}} \leq 1$, then $\lim _{m \rightarrow \infty} \mathbf{A}^{m}$ exists, and if no $\lambda_{\mathrm{s}}=1$, this limit is $O$.

Square Root of a Matrix Suppose the matrix A is diagonalizable with positive eigenvalues $\lambda_{1}, \ldots, \lambda_{n}$. Let $\mathbf{E}$ be a matrix, where eigenvectors are written as columns, and $\mathbf{D}$ is a diagonal matrix with $\lambda_{i}$ 's over diagonal, then

$$
D=\left(\begin{array}{cccc}
\lambda_{1} & 0 & \ldots & 0 \\
0 & \lambda_{2} & \cdots & 0 \\
\vdots & \vdots & \ddots & \vdots \\
0 & 0 & \cdots & \lambda_{n}
\end{array}\right)
$$

such that

$$
\mathbf{A}=\mathbf{E} \cdot \mathbf{D} \cdot \mathbf{E}^{-1}
$$

Let all $\lambda_{i}$ 's be nonnegative numbers. Now let's consider a matrix $\sqrt{\mathbf{D}}$ which has either positive or negative square root of $\lambda_{i}{ }^{\prime}$ s on diagonal:

$$
\sqrt{\mathbf{D}}=\left(\begin{array}{cccc} 
\pm \sqrt{\lambda_{1}} & 0 & \cdots & 0 \\
0 & \pm \sqrt{\lambda_{2}} & \cdots & 0 \\
\vdots & \vdots & \ddots & \vdots \\
0 & 0 & \cdots & \pm \sqrt{\lambda_{n}}
\end{array}\right)
$$

It can be easily checked that for such defined $\sqrt{\mathbf{D}}$, where $(\sqrt{\mathbf{D}})^{2}=\mathbf{D}$. So is demonstrated that a square root of a matrix $\mathbf{A}$ can be obtained from $\mathbf{D}$ and $\mathbf{E}$ by the following formula

$$
\mathbf{A} \cdot \mathbf{E}=\mathbf{E} \cdot \mathbf{D} \rightarrow \mathbf{A}=\mathbf{E} \cdot \mathbf{D} \cdot \mathbf{E}^{-1}
$$




$$
\sqrt{\mathbf{A}}=\mathbf{E} \cdot \sqrt{\mathbf{D}} \cdot \mathbf{E}^{-1}
$$

\section{Demonstration}

$$
\mathbf{A}=\sqrt{\mathbf{A}} \cdot \sqrt{\mathbf{A}}=\mathbf{E} \cdot \sqrt{\mathbf{D}} \cdot \mathbf{E}^{-1} \cdot \mathbf{E} \cdot \sqrt{\mathbf{D}} \cdot \mathbf{E}^{-1}
$$

where $\mathbf{E}^{-1} \cdot \mathbf{E}=\mathbf{I}$

$$
\mathbf{A}=\sqrt{\mathbf{A}} \cdot \sqrt{\mathbf{A}}=\mathbf{E} \cdot \sqrt{\mathbf{D}} \cdot \sqrt{\mathbf{D}} \cdot \mathbf{E}^{-1}=\mathbf{E} \cdot \mathbf{D} \cdot \mathbf{E}^{-1}
$$

Symbolic Development Being the matrix

$$
\mathbf{A}=\left(\begin{array}{ll}
a & b \\
c & d
\end{array}\right)
$$

The matrix of eigenvalues $\mathbf{D}$ corresponds with a diagonal matrix with the following expression:

$$
\mathbf{D}=\left(\begin{array}{cc}
\frac{a+d}{2}-\frac{\sqrt{a^{2}-2 a d+d^{2}+4 b c}}{2} & 0 \\
0 & \frac{a+d}{2}+\frac{\sqrt{a^{2}-2 a d+d^{2}+4 b c}}{2}
\end{array}\right)
$$

The obtained matrix of eigenvectors is shown in the next expression

$$
\mathbf{E}=\left(\begin{array}{cc}
\frac{a-d}{2}+\frac{\sqrt{a^{2}+d^{2}-2 a d+4 b c}}{2 c} & \frac{a-d}{2}-\frac{\sqrt{a^{2}+d^{2}-2 a d+4 b c}}{2 c} \\
1 & 1
\end{array}\right)
$$

Finally, the square root of matrix $\mathbf{A}$ is obtained with the following expression

$$
\sqrt{\mathbf{A}}=\left(\begin{array}{cc}
r_{\mathbf{A}}^{(11)} & r_{\mathbf{A}}^{(12)} \\
r_{\mathbf{A}}^{(21)} & r_{\mathbf{A}}^{(22)}
\end{array}\right)
$$

with $\gamma=\sqrt{a^{2}-2 a d+d^{2} 4 b c}$

$$
r_{\mathbf{A}}^{(11)}=\frac{1}{\gamma \cdot 2 \sqrt{2}}((a-d+\gamma) \sqrt{a+d+\gamma}+(d-a+\gamma) \sqrt{a+d-\gamma})
$$




$$
r_{\mathbf{A}}^{(12)}=\frac{1}{c \gamma 4 \sqrt{2}}((a-d+\gamma) \cdot(d-a+\gamma) \cdot \sqrt{a+d+\gamma}-(d-a+\gamma) \cdot(a-d+\gamma) \cdot \sqrt{a+d-\gamma})
$$

$$
\begin{gathered}
r_{\mathbf{A}}^{(21)}=\frac{c}{\gamma \cdot \sqrt{2}}(\sqrt{a+d+\gamma}-\sqrt{a+d-\gamma}) \\
r_{\mathbf{A}}^{(22)}=\frac{1}{\gamma \cdot 2 \sqrt{2}}((d-a+\gamma) \cdot \sqrt{a+d+\gamma}+(a-d+\gamma) \cdot \sqrt{a+d-\gamma})
\end{gathered}
$$




\section{Bibliography}

[1] A. Abdi and M. Kaveh. A Space-time Correlation Model for Multielement Antenna Systems in Mobile Fading Channels. IEEE Journal on Selected Areas in Communications, 20:550-560, 2002.

[2] A. Ahrens, F. Cano-Broncano, and C. Benavente-Peces. Power Distribution for SVD-aided MIMO Transmission with Receiver-Side Antennas Correlation. In 10th IASTED International Conference on Signal Processing, Pattern Recognition, and Applications (SPPRA) Innsbruck (Austria), pages 12 - 14, 2013.

[3] A. Ahrens, F. Cano-Broncano, and C. Benavente-Peces. Modulation-Mode and Power Assignment in SVD-assisted MIMO Systems with Transmitter-side Antennas Correlation. In X International Conference on Wireless Information Networks and Systems (WINSYS), Reykjavik (Iceland), 29-31 July 2013. Best Paper Award.

[4] A. Ahrens, S Kuhn, and T. Weber. Iterative Detection for Spatial Multiplexing with Adaptive Power Allocation. In 7th International Conference on Source and Channel Coding (SCC), Ulm, (Germany), pages 1-6, January 2008.

[5] A. Ahrens and C. Lange. Modulation-Mode and Power Assignment in SVDEqualized MIMO Systems. In 7th International Conference on Source and Channel Coding (SCC), 2008.

[6] Andreas Ahrens, Cesar Benavente-Peces, and Francisco Cano-Broncano. Power allocation in svd- and gmd-assisted mimo systems. Optimization and Engineering, pages 1-22, 2016.

[7] E. K. S. Au, S. Jin, M. R. McKay, W. H. Mow, X. Gao, and I. B. Collings. Ber analysis of mimo-svd systems with channel estimation error and feedback delay. In 2007 IEEE International Conference on Communications, pages 4375-4380, June 2007.

[8] C. Benavente-Peces, F. Cano-Broncano, A. Ahrens, FJ Ortega-Gonzalez, and JM Pardo. Analysis of Singular Values PDF and CCDF on Receiver-Side Antennas Correlated MIMO Channels. Electronics Letters, 49, Issue: 9:625-627, 2013. 
[9] F. Cano-Broncano, A. Ahrens, and C. Benavente-Peces. Adaptive Power Allocation in SVD-aided SDM-MIMO Systems. In XV International PhD Workshop OWD, pp 268-273, Wisla, 19-22 October 2013.

[10] F. Cano-Broncano, A. Ahrens, and C. Benavente-Peces. Power Allocation Techniques in MIMO Systems. In XV International PhD Workshop OWD, pp 274277, Wisla, 19-22 October 2013.

[11] F. Cano-Broncano, C. Benavente-Peces, A. Ahrens, F. Ortega, and JM Pardo. Analysis of MIMO Systems with Transmitter-side Antennas Correlation. In International Conference on Pervasive and Embedded Computing and Communication Systems (PECCS), Barcelona, (Spain), 19-21 February 2013.

[12] F. Cano-Broncano, C. Benavente-Peces, A. Ahrens, F. Ortega, and JM Pardo. Analysis of MIMO Systems with Antennas Correlation with Linear and Nonlinear Spatial Distribution. In International Conference on Pervasive and Embedded Computing and Communication Systems (PECCS), 24-26 S. 278-283, Rome, (Italy), 2012.

[13] J.B Carrel. Fundamentals of Linear Algebra. The University of British Columbia, 2005.

[14] M. Chiani, M.Z. Win, and A. Zanella. On the Capacity of Spatially Correlated MIMO Rayleigh-fading Channels. IEEE Transactions on Information Theory, 49:2363-2371, 2003.

[15] Y. Cho, J. Kim, W. Yang, and C. Kang. MIMO-OFDM Wireless Communications with Matlab. John Wiley \& Sons, 2010.

[16] S. S. Das and S. Batabyal. Comparison of svd-mimo with antenna-selectionblast using linear receivers under channel estimation errors for itu channels. In Vehicular Technology Conference (VTC Fall), 2011 IEEE, pages 1-5, Sept 2011.

[17] R. Fischer, C. Windpassinger, A. Lampe, and J. Huber. Tomlinson-Harashima precoding in space-time transmission for low-rate backward channel. In International Zurich Seminar on Broadband Communications, 2002.

[18] G.J Foschini. Layered Space-time Architecture for Wireless Communication in Fading Environments when using Multi-element Antennas. Bell Labs Tech, 1:4159, 1996.

[19] G.J Foschini and M.J. Gans. On limits on wireless communications in a fading environment when using multiple antennas. Wirel. Pers. Commun., 6:311-335, 1998.

[20] Goldfeld. Systems BER Power Loading for OFDM in Fading Channel. IEEE Transactions on Communications, 50:1729-1733, 2002. 
[21] A. Goldsmith, S.A. Jafar, N. Jindal, and S. Vishwanath. Capacity Limits of MIMO Channels. IEEE Journal on Selected Areas in Communications, 21:684$702,2003$.

[22] A. I, R. A. K, A. K. K, S. T C, A. Padmakumar, and R. R. A performance study of mimo detectors in the presence of channel estimation errors. In Communication, Information Computing Technology (ICCICT), 2015 International Conference on, pages 1-5, Jan 2015.

[23] R. Janaswamy. Effect of element mutual coupling on the capacity of fixed length linear arrays. IEEE Antennas and Wireless Propagation Letters, 1(1):157-160, 2002.

[24] Y. Jiang, W. Hager, and L. Jian. The Generalized Triangular Decomposition. Mathematics of Computation, 77:1037-1056, 2008.

[25] Y. Jiang, J. Li, and W. Hager. Joint Transceiver Design for MIMO Communications Using Geometric Mean Decomposition. IEEE Transactions on Signal Processing, 53:3791-3803, 2005.

[26] S. Kinjo and S. Ohno. A Tomlinson-Harashima precoding for correlated MIMO channels. In International Symposium on Intelligent Signal Processing and Communications Systems (ISPACS), 2013.

[27] B.S. Krongold, K. Ramchandran, and D.L. Jones. Computationally Efficient Optimal Power Allocation Algorithm for Multicarrier Communication Systems. IEEE Transactions on Communications, 48:23-27, 2000.

[28] V Kuhn. Wireless Communications over MIMO Channels. John Wiley \& Sons, 2006.

[29] S. Lin, W. Ho, and L. Ying-Chang. Block-Diagonal Geometric Mean Decomposition (BD-GMD) for Multiuser MIMO Broadcast Channels. In IEEE International Symposium on Personal, Indoor and Mobile Radio Communications, 2006.

[30] S. Loyka and G. Tsoulos. Estimating MIMO System Performance using the Correlation Matrix Approach. IEEE Communications Letters, 6:19 - 21, 2002.

[31] C.D Meyerm. Matrix Analysis and Applied Linear. SIAM, 2004.

[32] Leveque O. Ozgur, A. and D. Tse. Spatial degrees of freedom of large distributed mimo systems and wireless ad hoc networks. IEEE J. Sel. Areas Commun.,, $31: 202-214,2013$.

[33] J. Proakis and M. Saheli. Digital Communications. McGraw-Hill, 2008.

[34] D.-S. Shiu, Foschini G.J, M.J. Gans, and J.M Kahn. Fading Correlation and its effect on the Capacity of multi-element antenna systems. In Universal Personal Communications, 1998. 
[35] E. Telatar. Capacity of Multi-antenna Gaussian Channels. In Eur. Trans. Telecomm. ETT, 1999.

[36] D. Tse and P. Viswanath. Fundamentals Wireless Communications. Cambridge University Press, 2005.

[37] C-C. Weng, C-Y. Chen, and P.P Vaidyanathan. MIMO Tranceivers with Decision Feedback and Bit Loading: Theory and Optimization. IEEE Transactions on Signal Processing, 58:1334-1346, 2010.

[38] C. Windpassinger, R. Fischer, T. Vencel, and J. Huber. Precoding in multiantenna and multiuser communications. IEEE Transactions on Wireless Communications, 3:1305-1316, 2004.

[39] P. Yang, Y. Xiao, Y. Yu, and S. Li. Adaptive Spatial Modulation for Wireless MIMO Transmission Systems. IEEE Communications Letters, 15:602-604, 2011.

[40] A. Zanella and M. Chiani. Reduced Complexity Power Allocation Strategies for MIMO Systems With Singular Value Decomposition. IEEE Transactions on Vehicular Technology, 61:4031 - 4041, 2012.

[41] L. Zheng, G.L. Stuber, D. Wang, and Y. Cao. Dynamic Bound Restriction Iterative Algorithm Framework and Application to Power and Bit Allocation in OFDM Systems. IEEE Transactions on Communications, 61:1160-1170, 2013.

[42] Lizhong Zheng. Diversity and Multiplexing: A Fundamental Tradeoff in Multiple-antenna channels. IEEE Transactions on Information Theory, 49:10731096, 2003.

[43] Z. Zhou, B. Vucetic, M. Dohler, and Y. Li. MIMO Systems with Adaptive Modulation. IEEE Transactions on Vehicular Technology, 54:1828 - 1842, 2005. 